University of Rhode Island

DigitalCommons@URI

Open Access Dissertations

2017

\title{
An Investigation into the Influence of Interfacial Effects in a Three Layer Solid Phase Sensing Platform
}

Matthew J. Mullen

University of Rhode Island, mmullen@chm.uri.edu

Follow this and additional works at: https://digitalcommons.uri.edu/oa_diss

\section{Recommended Citation}

Mullen, Matthew J., "An Investigation into the Influence of Interfacial Effects in a Three Layer Solid Phase Sensing Platform" (2017). Open Access Dissertations. Paper 577.

https://digitalcommons.uri.edu/oa_diss/577

This Dissertation is brought to you for free and open access by DigitalCommons@URI. It has been accepted for inclusion in Open Access Dissertations by an authorized administrator of DigitalCommons@URI. For more information, please contact digitalcommons-group@uri.edu. 


\author{
AN INVESTIGATION INTO THE INFLUENCE OF \\ INTERFACIAL EFFECTS IN A THREE LAYER SOLID \\ PHASE SENSING PLATFORM \\ BY \\ MATTHEW J. MULLEN
}

A DISSERTATION SUBMITTED IN PARTIAL FULFILLMENT OF THE

REQUIREMENTS FOR THE DEGREE OF

DOCTOR OF PHILOSOPHY

IN

CHEMISTRY

UNIVERSITY OF RHODE ISLAND

2017 


\title{
DOCTOR OF PHILOSOPHY DISSERTATION
}

OF

MATTHEW J. MULLEN

\section{APPROVED:}

Dissertation Committee:

\author{
Major Professor $\quad$ William B. Euler \\ Sze C. Yang \\ Michael L. Greenfield \\ Nasser H. Zawia \\ DEAN OF THE GRADUATE SCHOOL
}

\section{UNIVERSITY OF RHODE ISLAND} 2017 


\begin{abstract}
With modern technological advances, we are seeing an increasing amount of electronic devices and materials going the route of smaller and faster. It has become of great importance to understand how these devices and materials work on the nanometer scale. Many of these new materials are coming to fruition in the form of thin films, and the topic of this dissertation focuses on the application of these materials to the field of chemical sensing. Chapter 1 of this dissertation investigates the photophysics of a thin film of the fluorescent dye, rhodamine 6G (Rh6G), on the surface of poly(vinylidene fluoride) (PVDF) coated glass, to determine the origin of a previously reported fluorescence enhancement. Three factors were identified that contribute to the increase in fluorescence seen in this system when compared to a thin film of Rh6G on a bare glass surface. First, the surface roughness of the underlying PVDF films provides a larger area for an excitation beam to interact with, which leads to the excitation of more Rh6G molecules. Second, the PVDF layer reduces the amount of aggregation between Rh6G molecules as the film thickness of the dye layer increase, which preserves the emissive monomeric form of Rh6G. Last, the PVDF thin films acts as a light trapping layer which leads to more Rh6G absorption events and more efficient use of the incident light.
\end{abstract}

Chapter 2 of this dissertation shows how the surface morphology of polystyrene (PS) thin films on a glass substrate evolves as the molecular weight $\left(\mathrm{M}_{\mathrm{w}}\right)$ of the PS used to cast the film is changed. Optical profilometry was used to collect images of the different films which revealed that PS pillars are formed in the center which transition into wrinkles that extend out from the center. The periodicity and 
amplitudes of these features changed with the $\mathrm{M}_{\mathrm{w}}$, which was found to be a result of the direct relationship between the glass transition temperature of PS and its $\mathrm{M}_{\mathrm{w}}$. Furthermore, this study showed a method of producing micrometer sized wrinkled interfaces spontaneously, which before required a more complicated process to fabricate.

The last chapter of this dissertation, chapter 3 , contains a spectroscopic study on thin films of differently charged xanthene dyes on the surface of PS coated glass to investigate potential ion- $\pi$ interactions. Coupling deconvolution of absorbance and emission spectra with measurement of excited state lifetimes of the dyes on the PS surface revealed that a large portion of monomer emission from the cationic dye (Rh6G) and the anionic dye (disodium fluorescein, DSF) was quenched by formation of a weakly emissive exciplex. This is in stark contrast to the neutral dye (fluorescein 27, F27), which showed no sign of exciplex formation and had the opposite emission behavior with respect to increasing the dye layer film thickness when compared to the charged dyes.

The overall findings of these studies show that there are complicated dynamics that can occur in solid phase, layered thin film systems. Interfacial interactions, whether they originate in physical or chemical nature, can have a vast effect on the performance of these devices. This dissertation demonstrates how having a fundamental understanding of such systems can reveal information necessary for the complete optimization of sensors and other nano-sized devices. 


\section{ACKNOWLEDGMENTS}

Without these people none of this would have been possible:

Bill Euler, for taking the time and having the patience to guide and help me grow as a scientist. Your wisdom, knowledge, and understanding of Chemistry and attention to detail will forever have an impact on the way I think about not only science, but life in general. You have been an outstanding mentor and advisor throughout this process and for that I will forever be grateful.

Rebecca Levine, my fiancée and forever the love of my life. The way that we have grown together throughout this time is something I will treasure for eternity. From day one, you have always been there for me emotionally and your intellect has always helped me find the right path. I simply could not have done this without you.

My family, for always believing in me no matter what and for laying the groundwork that has lead me to this point in my life. To my Father, you have taught me what it means to be an honorable, respectable man. As a child you were my role model and as an adult you are my best friend. To my Mother, you have taught me how to be responsible and loving. You have always pushed me to be my best and without your guidance and wisdom I never have made it to where I am today. To my Sister, for

your constant words of encouragement and motivation. You taught me how to be confident and to believe in myself.

To all my friends, old and new, without having you by my side to blow off steam I would have never got past day one of this process. 


\section{PREFACE}

The following dissertation is presented in manuscript format and the research is presented in three chapters. The first chapter is titled "The Influence of Interfacial Effect on the Photophysics of Rhodamine 6G Thin Films on a Poly(vinylidene fluoride) Surface" and is published in Langmuir. The second chapter is titled "Surface Morphology Dependence on Molecular Weight in Polystyrene Thin Films: Formation of Periodic Structures by Spin-Casting” and is in preparation for submission to Macromolecules. The last chapter of this dissertation is titled "A Spectroscopic Study of Charged Xanthene Dyes on a Polystyrene Surface: An Investigation into Potential Ion- $\pi$ Interactions" and is in preparation for submission to the Journal of Physical Chemistry $C$. 


\section{TABLE OF CONTENTS}

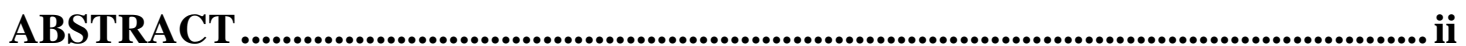

ACKNOWLEDGMENTS ............................................................................................. iv

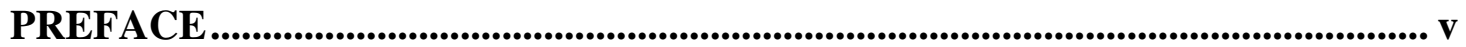

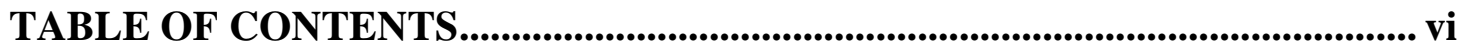

LIST OF TABLES ................................................................................................................... vii

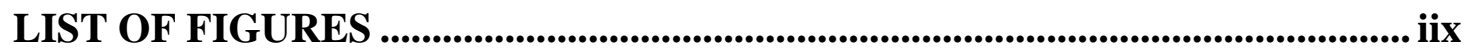

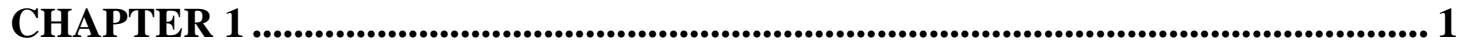

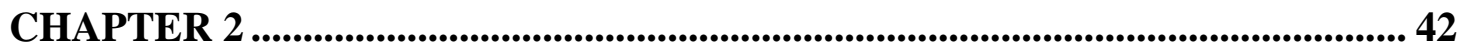

CHAPTER 3 ................................................................................................................. 76 


\section{LIST OF TABLES}

TABLE

PAGE

\section{Chapter 1:}

Table 1. Deconvoluted Gaussian peaks for the absorbance spectra. Peak position $\left(\lambda_{\max }\right)$ and FWHM $(\Gamma)$ have an uncertainty of $\pm 2 \mathrm{~nm}$. All spectra with fits and component

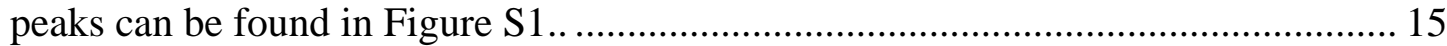

Table 2. Deconvolution parameters for excitation and emission spectra. Peak position $\left(\lambda_{\max }\right)$ and FWHM $(\Gamma)$ have an uncertainty of $\pm 3 \mathrm{~nm}$ 19

Table 3. Component contribution of the global multi-exponential fit for fluorescence decay curves their associated decay times as a function of $t_{\mathrm{Rh} 6 \mathrm{G}}, \mathrm{t}_{\mathrm{PVDF}}=760 \mathrm{~nm} . \alpha_{\mathrm{i}}$ is a pre-exponential term denoting its relative contribution of the $i^{\text {th }}$ exponential to the overall decay. $\tau_{\mathrm{i}}$ is the time constant for the excited state, or the value of $1 / e$ for the $i^{\text {th }}$ exponential. $\chi^{2}$ is an indication of how good the fit is and should be as close to a value of 1 as possible. The standard deviation in the lifetime is determined from two measurements

\section{Chapter 3:}

Table 1. Comparison of interaction strength based on peak separation 87

Table 2. Lifetimes and component contribution from decay fits. $\alpha_{\mathrm{i}}$ is the preexponential factor that shows the relative percentage that the $i^{\text {th }}$ component contributes to the overall decay. $\tau_{\mathrm{i}}$ represents the average lifetime of the $i^{\text {th }}$ component. $\chi^{2}$ is representative of how good the exponential fit is and should be as close to 1 as possible. 
Table S1. Deconvolution parameters for the absorbance and emission spectra of dye thin films on PS. $\lambda_{\max }$ represent the peak position and $\Gamma$ is the FWHM. Concentration range indicates the range in which the corresponding peak was required to fit the spectrum of the film cast from that concentration ..................................................... 116 


\section{LIST OF FIGURES}

FIGURE

PAGE

\section{Chapter 1:}

Figure 1. PVDF film thickness as a function of spin-casting rotation rate. The thicknesses were determined using reflection spectra ....................................... 8 Figure 2. Top: Surface morphology of a $760 \mathrm{~nm}$ thick PVDF film. Bottom: Average surface roughness as a function of PVDF film thickness. The surface roughness is calculated using the root-mean squared (RMS) method....................................... 9

Figure 3. FTIR spectrum for PVDF thin film; characteristic peaks show the crystalline

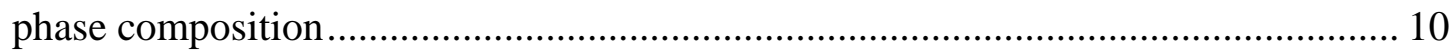

Figure 4. Absorbance spectra of Rh6G spun cast onto PVDF thin films .................. 12

Figure 5. Absorbance spectra as a function of PVDF film thickness ....................... 16

Figure 6. Absorbance intensity at $520 \mathrm{~nm}$ as a function of PVDF film thickness ...... 17

Figure 7. Steady-state emission spectra of different $t_{\mathrm{Rh} 6 \mathrm{G}}$ on $\mathrm{t}_{\mathrm{PVDF}}=760 \mathrm{~nm} \ldots \ldots \ldots \ldots 18$

Figure 8. Height normalized absorbance and excitation spectra (detected at $550 \mathrm{~nm}$ ) of

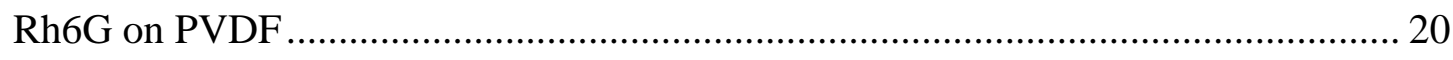

Figure 9. Steady-state fluorescence spectra as a function of PVDF film thickness.... 23

Figure 10. Lifetime decay curves of $\mathrm{t}_{\mathrm{Rh} 6 \mathrm{G}}=2.3 \mathrm{~nm}, 0.9 \mathrm{~nm}$, and $0.7 \mathrm{~nm} \ldots \ldots \ldots \ldots \ldots . . . . . .24$

Figure 11. Fluorescence spectra extracted from TRES data at selected times and $t_{\text {Rh6G }}$ with PVDF thickness $=760 \mathrm{~nm}$ 26

Figure S1. Fit lines constructed from the deconvolution of the absorbance spectra as a function of $t_{\text {Rh6G }}$. $t_{\mathrm{PVDF}}=760 \mathrm{~nm}$ 37 
Figure S2. Fit lines constructed from the deconvolution of the emission spectra as a function of $t_{\mathrm{Rh} 6 \mathrm{G}} \cdot \mathrm{t}_{\mathrm{PVDF}}=760 \mathrm{~nm}$ 39

Figure S3. Fit lines constructed from the deconvolution of the excitation spectra of various combination of $t_{\mathrm{PVDF}}$ and $\mathrm{t}_{\mathrm{Rh} 6 \mathrm{G}}$ 41

Figure S4. Spectral overlap of the deconvoluted peaks from the emission of monomeric Rh6G (light gray area, dashed blue line) with the absorbance of aggregated Rh6G (black area, solid red line)

\section{Chapter 2:}

Figure 1. Film thickness as a function of PS $M_{w}$. The data is fit to the equation $h=a x^{b}$, where

$\mathrm{b}=1 / 6$ 47

Figure 2. Polystyrene average film thickness as a function of spin speed for $\mathrm{M}_{\mathrm{w}}=$ $350000 \mathrm{~g} / \mathrm{mol}$. The solid line represents a fit of $\mathrm{h}=\mathrm{ax}{ }^{\mathrm{b}}$, where $\mathrm{b}=-1 / 2$. 48

Figure 3. Film thickness as a function of PS concentration spun at 1200 RPM for multiple molecular weights 48

Figure 4. Graphic displaying the location of the different types of structures on the PS sample 49

Figure 5. Top down view of select PS films of different molecular weight. Images are $2 \mathrm{~mm} \times 1.7 \mathrm{~mm}$ and collected at the center of the sample.

Figure 6. Top down view of select PS films of different molecular weight. Images are

$2 \mathrm{~mm} \times 1.7 \mathrm{~mm}$ and collected on one side of the sample. 50

Figure 7. Average A) Pillar wavelength, B) Pillar amplitude, C) Wrinkle Wavelength, 
and D) Wrinkle Amplitude as a function of polystyrene molecular weight. 52

Figure 8. 2D Schematic showing how the drying process leads to wrinkle formation in the PS thin films 54

Figure 9. Bulk glass transition temperature as a function of polystyrene molecular weight 55

Figure 10. A) Average wrinkle wavelength and amplitude as a function of spin speed for a $2.0 \%$ PS solution. B) Average wrinkle wavelength and amplitude as a function of PS concentration, films spun at 1200 RPM. Polystyrene $M_{w}=350 \mathrm{~kg} / \mathrm{mol}$ for both A and $\mathrm{B}$ 57

Figure S1. Line scan profiles of the wrinkled polystyrene surface. 70

Figure S2. Line scan profiles of the pillared polystyrene surface. 75

\section{Chapter 3:}

Figure 1. Molecular structure of fluorophores and polymer 79

Figure 2. Absorbance spectra of A) Rh6G, C) F27, and E) DSF as a function of casting concentration. B, D, and F, are height normalized version of the same spectra, respectively

Figure 3. Dilute $\left(10^{-6} \mathrm{M}\right)$ absorbance and emission spectra of A) Rh6G, B) F27, and C) DSF dissolved in EtOH 84

Figure 4. Emission spectra and height normalized emission spectra as a function of casting concentration of A) \& B) Rh6G, C) \& D) F27, and E) \& F) DSF 91

Figure 5. Fluorescence lifetime decays of various casting concentrations of A) Rh6G $\left(\lambda_{\mathrm{em}}=550 \mathrm{~nm}\right)$, B) F27 $\left(\lambda_{\mathrm{em}}=540 \mathrm{~nm}\right)$, and C) DSF $\left(\lambda_{\mathrm{em}}=540 \mathrm{~nm}\right) . \lambda_{\mathrm{ex}}=452 \mathrm{~nm}$ 
Figure S1. Deconvoluted absorbance spectra of films cast from different Rh6G

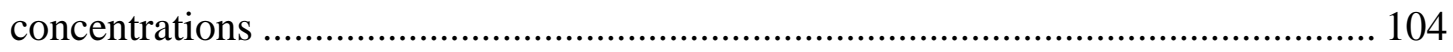

Figure S2. Deconvoluted absorbance spectra of films cast from different F27

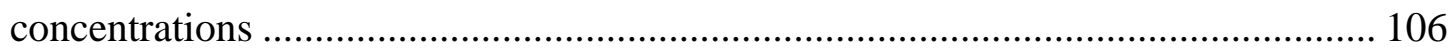

Figure S3. Deconvoluted absorbance spectra of films cast from different DSF

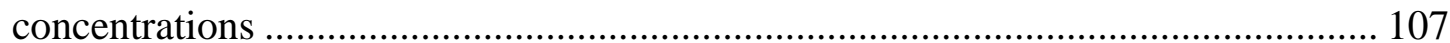

Figure S4. Deconvoluted emission spectra of films cast from different Rh6G concentrations 109

Figure S5. Deconvoluted emission spectra of films cast from different F27 concentrations 110

Figure S6. Deconvoluted emission spectra of films cast from different DSF concentrations 112

Figure S7. Deconvoluted spectra of $[\mathrm{Rh} 6 \mathrm{G}]=1.0 \times 10^{-6} \mathrm{M}$ in EtOH. Top: absorbance,

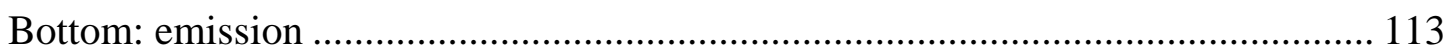

Figure S8. Deconvoluted spectra of $[\mathrm{F} 27]=1.0 \times 10^{-6} \mathrm{M}$ in EtOH. Top: absorbance, Bottom: emission 114

Figure S9. Deconvoluted spectra of $[\mathrm{DSF}]=1.0 \times 10^{-6} \mathrm{M}$ in EtOH. Top: absorbance, Bottom: emission 115 


\title{
CHAPTER 1
}

The Influence of Interfacial Effects on the Photophysics of Rhodamine 6G Thin Films on a Poly(vinylidene fluoride) Surface

\author{
Matthew Mullen and William B. Euler* \\ Department of Chemistry, University of Rhode Island, 140 Flagg Road, Kingston, \\ Rhode Island 02881, United States
}

The following is published in Langmuir and is presented here in manuscript format. 


\begin{abstract}
The spectral response of ultrathin films of rhodamine 6G (Rh6G) cast onto poly(vinylidene fluoride) (PVDF) coated glass slides is studied to investigate a perceived fluorescence emission enhancement. Varying the thickness of the Rh6G layer (submonolayer to multiple layers) on the PVDF layer revealed the existence of multiple Rh6G species on the surface, similar to previous reports on glass. Excitation spectra show that J-type excitons are not responsible for an emission enhancement. Trends in the fluorescence emission intensity show that the surface roughness of the PVDF layer prevents the Rh6G molecules from organizing the way they would on a smooth glass surface. The PVDF surface roughness preserves the emissive monomers and excited-state excimers of Rh6G while reducing the self-quenching of aggregates. Coupled with this is an internal reflection effect that causes light to be trapped between the Rh6G/PVDF and PVDF/glass interfaces. This effect leads to multiple absorption events, and thus more efficient use of the incident light.
\end{abstract}

\title{
Introduction
}

The use of fluorescence to study the polarity of the surrounding environment is textbook material. ${ }^{1}$ This methodology is typically applied to solutions, either liquid or solid. Application to interfaces to determine surface polarity has also been reported. ${ }^{2-8}$ However, the structure of many fluorophores is dependent on the thickness or surface concentration, ${ }^{9,10}$ which can mask the interpretation of the substrate properties. The xanthene dyes are examples of this because they are susceptible to dimerization and higher-order aggregation, both of which have a significant effect on the photophysics 
of the dye. ${ }^{11-20}$ The focus in this Article will be the use of rhodamine 6G (Rh6G), a xanthene dye used for a variety of applications, ${ }^{21-25}$ to study the surface properties of poly(vinylidene fluoride) (PVDF). In work published by our research group, it was found that when an array of fluorescent dyes are independently dissolved in $\mathrm{N}, \mathrm{N}$ dimethylformamide (DMF), their emission can be greatly affected by molecules regularly used in military explosives and improvised explosive devices (IEDs). ${ }^{24}$ Depending on the combination of analyte and fluorophore, the emission was either enhanced or quenched, leading to fingerprint like detection and identification of the energetic analytes. To further investigate the extent of this type of detection, a solidphase sensing element was constructed using thin films of Rh6G deposited on polymer-coated substrates to detect $\mathrm{TNT}^{26}$ It was found that not only does this sensing element detect TNT in the gas phase efficiently, but adding the PVDF layer between the dye and substrate caused a 100-1000 times enhancement in the emission of Rh6G, depending on the substrate beneath the PVDF, when compared to samples that had Rh6G deposited directly onto substrate. To exploit this enhancement in signal, an array of thin films of other xanthene dyes was prepared on PVDF-coated glass microscope slides and was exposed to a variety of explosive analytes. ${ }^{25}$ The three-layer system of dye/PVDF/glass was found to respond similarly to the xanthene dyes in DMF solution in terms of that some fluorophores were quenched and others enhanced, depending on the fluorophore/analyte combination. The difference between the two methods was the magnitude of quenching or enhancement. In solution, most analytes only caused an $\sim 10 \%$ change in the emission of fluorophores one way or the other with the exceptions being TNT and TNB, but the solid phase thin films of 
fluorophores had a much stronger change for all gas-phase analytes. For example, in solution, 2,3- dinitrotoluene (2,3-DNT) causes a 4-6\% change in the emission of Rh6G, but when the thin film of Rh6G was exposed to 2,3-DNT vapors, the emission is quenched by nearly $100 \%$. A surprising result from the aforementioned study was that the response to an analyte could give either quenching or enhancement of the emission, depending on the film thickness of the underlying PVDF layer. To understand the dynamics of the Rh6G response, a detailed study was done with varying the Rh6G layer film thickness on the glass substrate and observing the absorbance and fluorescence responses. ${ }^{10}$ Using different concentrations of the Rh6G solution allowed for control over the thickness of the Rh6G layer, which ranged from submonolayer films to tens of layers. PVDF $\left([\mathrm{CH} 2 \mathrm{CF} 2]_{\mathrm{x}}\right)$ is interesting because of its unusual phase behavior. ${ }^{27-34}$ Depending upon the relative orientation of the C-F and C$\mathrm{H}$ bonds, the polymer can crystallize in the nonpolar $\alpha$-phase, the polar $\gamma$-phase, or the ferroelectric $\beta$-phase.

The purpose of this study is to investigate the role that the PVDF layer has on the previously reported emission enhancement. ${ }^{26}$ We report the photophysical behavior of Rh6G deposited onto PVDF films as a function of both the Rh6G thickness and the PVDF thickness. The observed emission enhancement of Rh6G when deposited on the polymer film as compared to a glass substrate arises from three sources. First, the PVDF films are rougher than glass substrates so that there are more molecules of Rh6G per unit area on PVDF than on a smooth surface. Second, the PVDF reduces the amount of aggregation of Rh6G, thereby reducing the amount of self-quenching. Finally, there are internal reflections of the incident light trapped in 
the PVDF layer, which allows for more opportunities for the light to be absorbed by the Rh6G layer.

\section{Experimental Methods}

Microscope glass slides were cut into dimensions of $3.75 \mathrm{~cm} \times 1.75 \mathrm{~cm}$. Once cut, the samples were placed in a container of 95\% ethanol (EtOH, Pharmaco-Aaper) and sonicated for 15 min. The glass slides were rinsed and placed in water and sonicated for an additional $15 \mathrm{~min}$, and then dried with $\mathrm{N}_{2}$ gas. A 4\% (w/v) solution of poly(vinylidene fluoride) (PVDF) (Sigma-Aldrich, $\mathrm{M}_{\mathrm{w}} \approx 534000$ ) was made in a 9:1 ratio of acetone (Fisher, HPLC grade) and N, N-dimethylformamide (DMF, Fisher, spectral grade) and sonicated for $3 \mathrm{~h}$ in a $40{ }^{\circ} \mathrm{C}$ bath to ensure all PVDF was in solution. A series of Rh6G chloride (Acros, 99\%) concentrations were made in EtOH ranging from $10^{-2} \mathrm{M}$ to $10^{-6} \mathrm{M}$. Each solution was wrapped in aluminum foil to keep out ambient light and reduce degradation of the Rh6G.

The PVDF solution was applied to the glass substrate by spin-casting. First, the glass slide sample was subjected to a $1 \mathrm{~min} \mathrm{~N}_{2}$ purge to reduce the effects of humidity

on the polymer. ${ }^{27} \mathrm{~A} 500 \mu \mathrm{L}$ aliquot of solution was placed on the sample and allowed to spread to the edge. At this point, the sample was spun at a given RPM for $45.0 \mathrm{~s}$ (acceleration of $1080 \mathrm{~s}^{-2}$ ). The sample was then placed in an oven set to $60{ }^{\circ} \mathrm{C}$ for 1 min to dry. The Rh6G solution was also spun-cast in a similar manner. A $250 \mu \mathrm{L}$ aliquot of solution was deposited on the sample and allowed to spread out over the slide. The sample was then spun at $1800 \mathrm{rpm}$ for $45.0 \mathrm{~s}$ (acceleration of $1080 \mathrm{~s}^{-2}$ ) and left to air dry. 
The film thickness of the PVDF was determined using a Filmetrics F40 microscope via reflection interference patterns and fitting software. The reflection spectrum was recorded with a tungsten-halogen light source over the range of 400-900 $\mathrm{nm}$ and then compared to a calculated spectrum of given thickness and refractive index. An Agilent Technologies 5500 AFM was used to collect images of the surface using tapping mode. Infrared spectroscopy was performed on an ATR equipped PerkinElmer Spectrum 100. Spectra were collected from 4000 to $650 \mathrm{~cm}^{-1}$. The absorbance spectrum of each sample was collected on a PerkinElmer Lambda $1050 \mathrm{UV} /$ vis/ NIR spectrometer with $2.0 \mathrm{~nm}$ slit widths. Before the Rh6G layer was applied to the sample, a blank of that sample with only the glass and PVDF layer was taken. This method proved to give the cleanest spectrum of the sample due to the minor inconsistencies in the PVDF on each slide. Steady-state fluorescence measurements were made on a Horiba (JobinYvon) Fluorolog spectrometer and collected at an angle of $60^{\circ}$. A xenon arc lamp excitation source at a wavelength of $520 \mathrm{~nm}$ was used to irradiate the samples. The emission range was set to $530-800$ $\mathrm{nm}$ with slit widths of $3.0 \mathrm{~nm}$ for both the light source and the detector. Excitation spectra were collected on the same instrument. The excitation range consisted of two scans, one from $350 \mathrm{~nm}$ to $\left(\lambda_{\max }-10 \mathrm{~nm}\right)$ and a second from $\left(\lambda_{\max }+10 \mathrm{~nm}\right)$ to 750 $\mathrm{nm}$ while monitoring fluorescence at a selected wavelength. Time-resolved emission spectroscopy (TRES) measurements were made using a Horiba Fluorohub timecorrelated single photon counting (TCSPC) system. A Horiba NanoLED N-460 pulsed diode was used with a wavelength of $464 \mathrm{~nm}$, a repetition rate of $1.0 \mathrm{MHz}$, and $160 \mathrm{ps}$ pulse duration time with a power output of about $7 \mathrm{pJ} / \mathrm{pulse}$. The instrumental 
response function (IRF) is recorded using a scattering solution. For each TRES experiment, a decay was collected every $1 \mathrm{~nm}$ from 500 to $630 \mathrm{~nm}$. The peak preset was set to 10000 counts. Global multiexponential fitting analysis was used to determine the lifetimes and pre-exponential factors of each component contributing to the emission. A separate steady-state fluorescence spectrum was collected for the TRES samples with excitation of $464 \mathrm{~nm}$ because that was the wavelength of the laser used for the TRES.

\section{Results and Discussion}

PVDF films were all produced by spin-casting a 4\% PVDF (w/ v) onto a glass slide. Different thicknesses were obtained by changing the rotation rate, and for this study the thickness range of the PVDF layer is $380-770 \mathrm{~nm}$. This thickness range was chosen to optimize light trapping of visible light. The measured thicknesses of the PVDF thin films as a function of rotation rate can be seen in Figure 1 determined from both the center and the edges of the films. The edges of the films tend to be thicker than the centers of the films for lower rotation rates. At higher rotation rates, the films are more uniform across the substrate. As noted by the small error bars in Figure 1, the reproducibility of the spin-coating is excellent. Atomic force microscope scans were performed on some of the neat PVDF films, and the thicknesses at the edge matched those measured by reflection spectroscopy. Figure 2 shows an AFM image of a 760 nm thick PVDF film. The surface roughness is notable so we measured this parameter as a function of PVDF film thickness. The results are shown in Figure 2. For thinner PVDF films, the surface roughness is a significant fraction of the average thickness, 
which suggests that there may be pinholes on the surface that expose the glass substrate. When the film thickness exceeds $\sim 450 \mathrm{~nm}$, the surface roughness is fairly constant at $\sim 175 \mathrm{~nm}$.

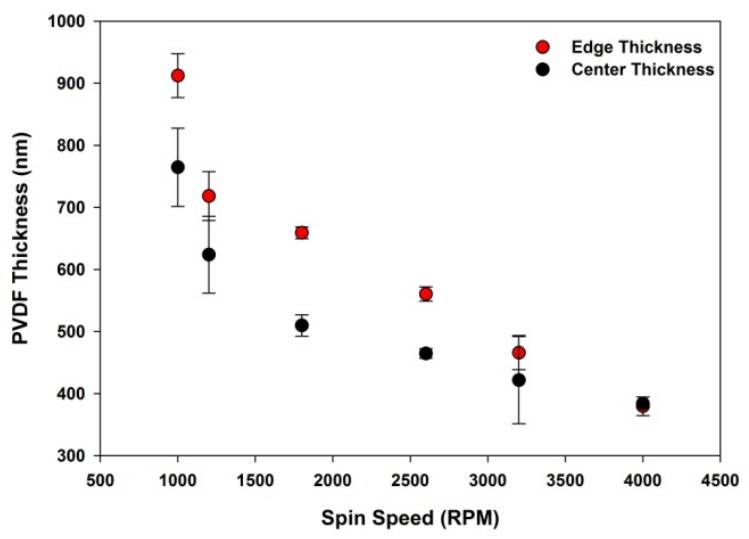

Figure 1. PVDF film thickness as a function of spin-casting rotation rate. The thicknesses were determined using reflection spectra. The error bars represent the standard deviation of six measurements for each spin speed.

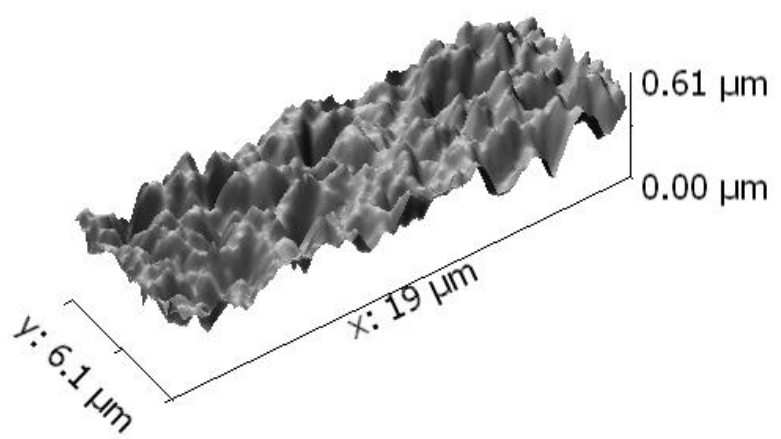




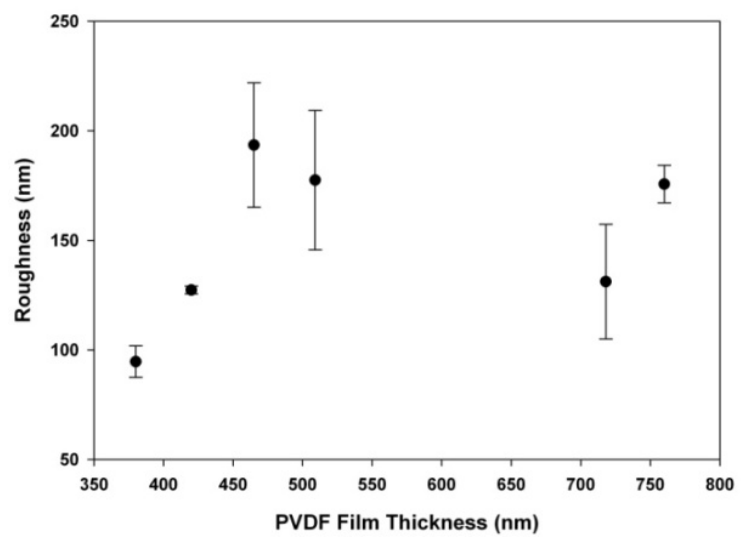

Figure 2. Top: Surface morphology of a $760 \mathrm{~nm}$ thick PVDF film. Bottom: Average surface roughness as a function of PVDF film thickness. The surface roughness is calculated using the root-mean squared (RMS) method. The error bars represent the standard deviation of three measurements for each thickness.

FTIR spectroscopy has been shown to be a convenient and accurate method to determine the phase composition of PVDF, correlating directly with XRD. ${ }^{29,37-40}$ FTIR spectra of PVDF coated on glass and PVDF scraped from a glass slide can be seen in Figure 3. The peaks are poorly resolved for the coated glass because the glass substrate interferes, but for the PVDF scraped from a glass slide the phases can be determined. The peak at $1286 \mathrm{~cm}^{-1}$ is indicative of $\beta$-phase and is assigned as a combination of $v_{\mathrm{s}}(\mathrm{CF} 2), v_{\mathrm{s}}(\mathrm{CC})$, and $\delta(\mathrm{CCC})$ modes. ${ }^{29,33,34,37-40}$ The $840 \mathrm{~cm}^{-1}$ peak is characteristic of both the $\beta$ - and the $\gamma$-phases and is assigned to the $\mathrm{r}(\mathrm{CH} 2)$ and $v_{\mathrm{a}}(\mathrm{CF} 2)$ modes. ${ }^{29,33,34,37-40}$ The other unique $\gamma$-phase peak is located at $1234 \mathrm{~cm}^{-1}$, which corresponds to the $v_{a}(\mathrm{CF} 2), r(\mathrm{CF} 2)$, and $r(\mathrm{CH} 2)$ modes. ${ }^{29,33,34,37-40}$ The peak at $760 \mathrm{~cm}^{-1}$ is present only in the $\alpha$-phase and is assigned to $\delta(\mathrm{CF} 2)$ and $\delta(\mathrm{CCC})$ vibrational modes. ${ }^{29,33,34,37-40}$ All of the phase sensitive peaks are observed, indicating that all three phases are present in the samples used in this study. 


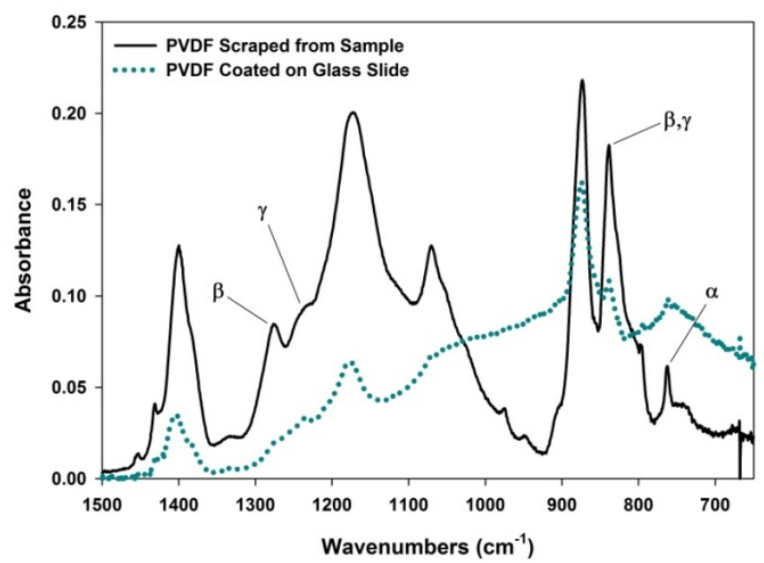

Figure 3. FTIR spectrum for PVDF thin film; characteristic peaks show the crystalline phase composition.

Rh6G films were spin-cast onto the PVDF films using EtOH as a solvent. EtOH does not dissolve PVDF so the Rh6G deposition does not alter the polymer film. The thickness of the Rh6G layer was varied by changing the concentration of the casting solution over 3 orders of magnitude, from $1 \times 10^{-5}$ to $1 \times 10^{-2} \mathrm{M}$, because changing the rotation rate was previously shown to have only a small effect on the Rh6G thickness. ${ }^{10}$ Because of the surface roughness of the PVDF, direct measurement of the Rh6G thickness using reflection spectroscopy was not possible. Instead, we used absorption spectroscopy (correcting for the scattering background) and the formulas given in ref 10 to estimate the Rh6G film thicknesses. These thicknesses are approximate with errors estimated to be $\pm 10 \%$, because the equations do not account for surface roughness. The nominal thicknesses ranged from 0.7 to $15.6 \mathrm{~nm}$. While the term thickness is used for submonolayer coverage $(<\sim 1.4 \mathrm{~nm})$, the correct usage would be surface density or number density. Thus, for a film with a nominal thickness 
of $0.7 \mathrm{~nm}$, the surface coverage is $\sim 50 \%$. We continue to use thickness with the understanding that it must be interpreted differently for different coverages.

The absorption spectra of various thicknesses of Rh6G, $t_{\mathrm{Rh} 6 \mathrm{G}}$, deposited on a $760 \mathrm{~nm}$ PVDF film are shown in Figure 4. At the lowest casting concentration where $[\mathrm{Rh} 6 \mathrm{G}]=1.0 \times 10^{-5} \mathrm{M}$ and $\mathrm{t}_{\mathrm{Rh} 6 \mathrm{G}}$ is at submonolayer thickness, $0.7 \mathrm{~nm}$, the absorption maximum is at $518 \mathrm{~nm}$. Increasing the casting concentration by an order of magnitude only changes the nominal thickness to $0.9 \mathrm{~nm}$, but the absorption spectrum broadens significantly while the peak is still at $518 \mathrm{~nm}$. When the casting concentration and $t_{\mathrm{Rh} G \mathrm{G}}$ are increased more, there is the gradual development of a new absorption peak indicating a new species/geometry of Rh6G on the surface that does not exist at the low film thicknesses. The peak appears in the region of $550-560 \mathrm{~nm}$ and shifts slightly to higher wavelengths with increasing Rh6G film thickness. This feature has been identified with aggregation and/or an exciton. ${ }^{10,12,14-16}$ A similar effect is observed on glass when the Rh6G film thickness is varied, but with one significant difference. 

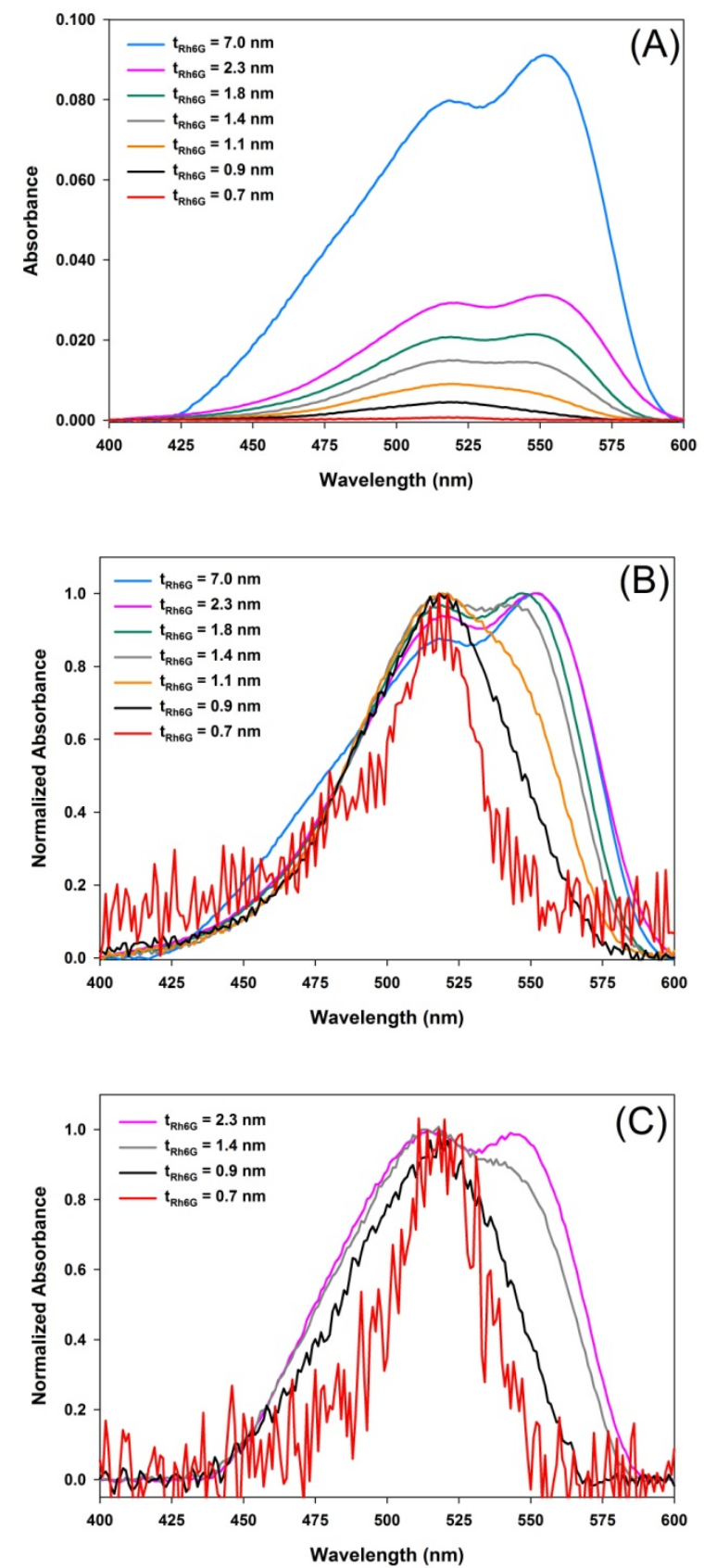

Figure 4. Absorbance spectra of Rh6G spun cast onto PVDF thin films. A) Changing the Rh6G thickness on constant PVDF film thickness $(760 \mathrm{~nm})$. B) Height normalized plot of A. C) Changing the Rh6G thickness on constant PVDF film thickness (380 $\mathrm{nm})$. 
On the glass substrate, the maximum absorbance wavelength changes gradually as a function of $t_{\mathrm{Rh} 6 \mathrm{G}}$, with the $\sim 520 \mathrm{~nm}$ component decreasing and the $\sim 560 \mathrm{~nm}$ feature increasing as $t_{R h 6 G}$ increases. On the PVDF surface, the peak at $518-521 \mathrm{~nm}$ remains the most intense until the Rh6G film thickness changes from 1.4 to $1.8 \mathrm{~nm}$. At this point, the most intense peak changes to $\sim 560 \mathrm{~nm}$, but the intensity of the $\sim 520 \mathrm{~nm}$ peak does not decrease. As the Rh6G film thickness grows from 0.9 to $2.3 \mathrm{~nm}$, both the populations of monomer Rh6G and aggregated/crystalline Rh6G grow concurrently until eventually the aggregated/ crystalline Rh6G species dominates at multilayer Rh6G thicknesses $(\mathrm{t} \geq 7 \mathrm{~nm})$. This implies that there are regions on the PVDF surface that prevent aggregation. Whether this is imposed by the surface morphology or electronic effects by regions of different phase is not clear. When the thickness of the Rh6G layer is changed on thin PVDF (380 nm), the resulting spectral changes are similar, as shown in Figure 4C. Deconvolution of the Rh6G spectra on $\mathrm{t}_{\mathrm{PVDF}}=380 \mathrm{~nm}$ leads to the same peak positions and shapes used to deconvolute the spectra on $t_{\mathrm{PVDF}}=760 \mathrm{~nm}$. Therefore, the PVDF thickness has little effect on the electronic properties of Rh6G and that the composition of the surface phases of the PVDF is similar for all thicknesses.

Deconvolution of the absorbance spectra (shown in Figure S1 and Table 1) shows the strong dependence that the Rh6G film thickness has on the amount and type of Rh6G species present on the PVDF surface. At submonolayer thicknesses, the absorbance spectrum consists of a main peak at $518 \pm 2 \mathrm{~nm}$ with a small shoulder to the high energy side at $495 \pm 2 \mathrm{~nm}$, and a very small shoulder on the low energy side at $542 \pm 2 \mathrm{~nm}$. These features are assigned to monomer Rh6G (518 nm) and an 
oblique exciton dimer (495 and $542 \mathrm{~nm}$ ) formed between two Rh6G molecules in the excited state, respectively. Two peaks arise due to the structure of oblique exciton dimers as described by Kasha. ${ }^{12}$ Increasing the Rh6G film thickness to $0.9 \mathrm{~nm}$ causes a change in the intensities of the peaks mentioned above, but does not require any additional peaks to fit. It is not until $\mathrm{t}_{\mathrm{Rh} 6 \mathrm{G}}=1.1 \mathrm{~nm}$ that an addition peak at $560 \pm 2 \mathrm{~nm}$ is needed to fit the spectrum. The new peak is assigned to a higher order aggregate of Rh6G that can be formed due to the amount of neighboring Rh6G molecules in confined areas on the PVDF surface. Last, when $\mathrm{t}_{\mathrm{Rh} 6 \mathrm{G}} \geq 7.0 \mathrm{~nm}$, a fifth peak at $452 \pm 2$ $\mathrm{nm}$ is needed to achieve a good fit. An isolated molecule of Rh6G has approximately C2 symmetry about the center of the middle aromatic ring in the xanthene plane, which means that the only transition moment that is allowed is the one across the long axis of the xanthene plane. However, as Rh6G molecules aggregate, the C2 symmetry is reduced and the transitions forbidden in the isolated molecule become allowed. Therefore, it is likely that the peak at $452 \pm 2 \mathrm{~nm}$ is originating from a transition moment perpendicular to the strongly allowed transition of the isolated molecule. Each of the peak maxima found for Rh6G on PVDF are blue shifted as compared to the parameters reported for Rh6G on glass. ${ }^{10}$ This indicates that the surface polarity of the PVDF is less than the glass surface, probably being dominated by the nonpolar $\alpha$-phase, which has a dielectric environment different from glass. 
Table 1. Deconvoluted Gaussian peaks for the absorbance spectra. Peak position $\left(\lambda_{\max }\right)$ and FWHM $(\Gamma)$ have an uncertainty of $\pm 2 \mathrm{~nm}$. All spectra with fits and component peaks can be found in Figure S1.

\begin{tabular}{|c|c|c|c|}
\hline Peak & $\lambda_{\max }(\mathbf{n m})$ & $\boldsymbol{\Gamma}(\boldsymbol{n m})$ & $\boldsymbol{t}_{\text {Rh6G }}(\mathbf{n m})$ \\
\hline 1 & 495 & 27 & $0.7-7.0$ \\
\hline 2 & 518 & 16 & $0.7-7.0$ \\
\hline 3 & 542 & 16 & $0.7-7.0$ \\
\hline 4 & 560 & 14 & $2.3-7.0$ \\
\hline 5 & 452 & 16 & 7.0 \\
\hline
\end{tabular}

The magnitude of the shift is greatest for the monomer absorption, which are $518 \mathrm{~nm}$ on PVDF and $527 \mathrm{~nm}$ on glass. The oblique exciton dimer peaks only shift 5-6 nm (495 and $542 \mathrm{~nm}$ on PVDF vs. 500 and $548 \mathrm{~nm}$ on glass), and the absorption assigned to the aggregate barely changes (560 nm on PVDF vs. $562 \mathrm{~nm}$ on glass). On an energy scale, the monomer shifts $330 \mathrm{~cm}^{-1}$, the dimer shifts $202 \mathrm{~cm}^{-1}$, and the aggregate shifts $64 \mathrm{~cm}^{-1}$. The monomer must reside directly adjacent to the surface so it is most strongly influenced by the substrate. The dimer and aggregate are less influenced by the surface because large portions of the chromophore are further from the polar environment.

The effect of the PVDF film thickness at constant Rh6G thickness was studied by preparing a set of PVDF films with thicknesses between 380 and $760 \mathrm{~nm}$, which were all then spun-cast with the same concentration of Rh6G, giving all samples the same average $t_{\mathrm{Rh} 6 \mathrm{G}}, \sim 2.3 \mathrm{~nm}$. As shown in Figure 5, the general shape of the Rh6G absorbance spectrum does not change much as a function of the PVDF film thickness. As the PVDF layer gets thicker, the 560 and $518 \mathrm{~nm}$ peaks fluctuate a small amount. 
As stated earlier, the PVDF film can act as a trapping mechanism for light via internal reflection between the Rh6G/PVDF and PVDF/Glass interfaces.

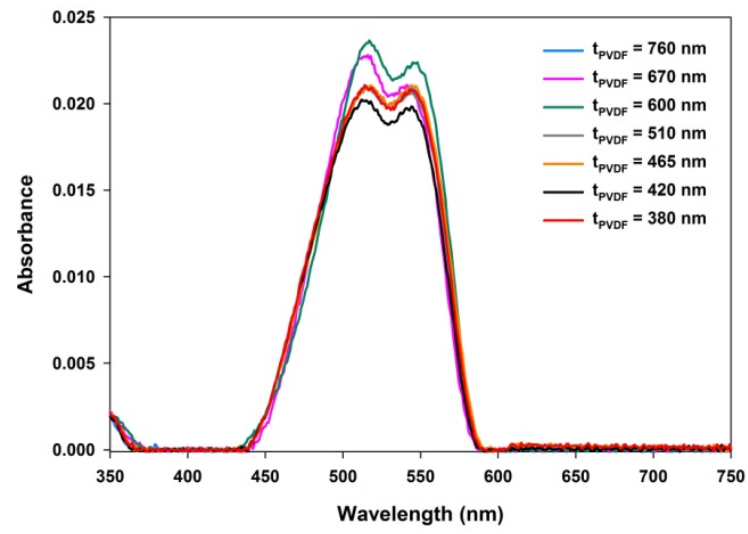

Figure 5. Absorbance spectra as a function of PVDF film thickness. The Rh6G thickness was constant at approximately $2.3 \mathrm{~nm}$.

Figure 6 shows the absorbance at $550 \mathrm{~nm}$ as a function of the thickness of the PVDF layer for two different Rh6G thicknesses. As expected for internal reflections between the different layers, there is a small intensity modulation in the Rh6G absorbance as the PVDF thickness changes. Elementary considerations using the Fresnel equations ${ }^{41}$ for normal incidence would predict extrema at $t_{\mathrm{PVDF}}=\mathrm{m} \lambda / 2 \mathrm{n}_{\mathrm{PVDF}}$ and $t_{\mathrm{PVDF}}=(\mathrm{m}-1 / 2) \lambda / 2 \mathrm{n}_{\mathrm{PVDF}}$, where $\mathrm{n}_{\mathrm{PVDF}}$ is the refractive index of the polymer $(\sim 1.4), \lambda$ is the wavelength of the incident light $(550 \mathrm{~nm}), \mathrm{t}_{\mathrm{PVDF}}$ is the polymer thickness, and $\mathrm{m}$ is an integer. This predicts that the maxima and minima should be about $98 \mathrm{~nm}$ apart as a function of PVDF thickness. Experimentally, it is observed that the difference is about $100 \mathrm{~nm}$, independent of the Rh6G thickness, which matches well with theory. 

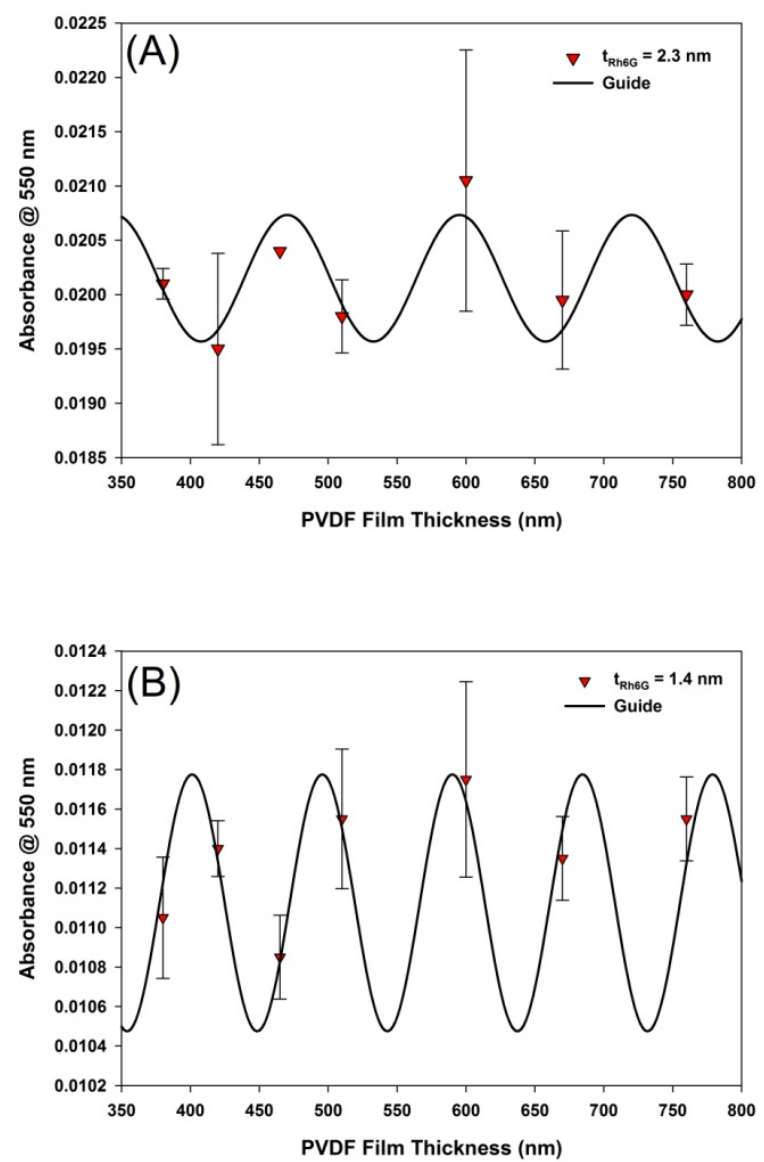

Figure 6. Absorbance intensity at $550 \mathrm{~nm}$ as a function of PVDF film thickness. A) $t_{\mathrm{Rh} 6 \mathrm{G}}=2.3 \pm 0.1 \mathrm{~nm}$. B) $t_{\mathrm{Rh} 6 \mathrm{G}}=1.4 \pm 0.1 \mathrm{~nm}$. The solid line is a fit to a sine function intended to be a guide for the eye. The error bars represent the standard deviation of two measurements for each thickness.

The emission spectra of several thicknesses of Rh6G on thick (760 nm) PVDF are shown in Figure 7A. As the Rh6G becomes thicker, two effects are observed: the spectra become more intense, and the peak maximum shifts to lower energy. At low Rh6G film thicknesses on PVDF, the emission maximum is narrower and positioned at $\sim 537 \mathrm{~nm}$, but as the thickness increases the maximum shifts to $555 \mathrm{~nm}$, as shown in Figure 7B. Moreover, as shown in Figure 7C, the spectra broaden so that the total intensity of the emission increases as the Rh6G thickness increases up to about $2 \mathrm{~nm}$ 
and then slowly decreases. This is in contrast to a glass substrate where the emission intensity increases up to a nominal thickness of about $2.3 \mathrm{~nm}$ and then drops precipitously, ${ }^{10}$ also shown in Figure 7C.
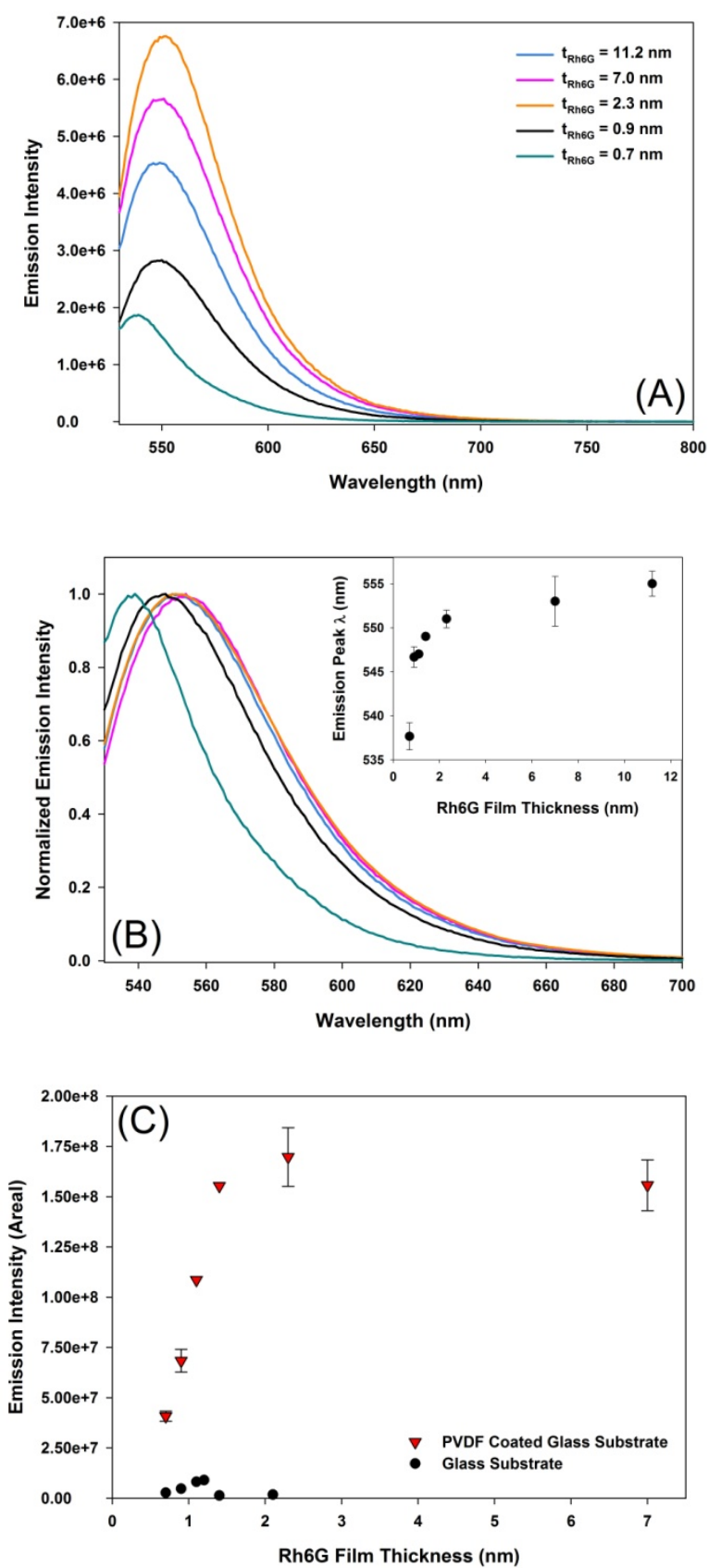

Figure 7. Steady-state emission spectra of different $t_{\text {Rh6G }}$ on $t_{P V D F}=760 \mathrm{~nm}$. A) Emission spectra as a function of $t_{\text {Rh6G }}$. B) Height normalized plot of A, insert shows 
wavelength dependence of $t_{\mathrm{Rh} 6 \mathrm{G}}$. C) Emission intensity as a function of $t_{\mathrm{Rh} 6 \mathrm{G}}$. The error bars represent the standard deviation of four measurements for each thickness.

Deconvolution of the emission spectra as a function of Rh6G film thickness (given in Figure S2 and Table 2) showed complementary data in agreement with the deconvoluted absorbance spectra. At the lowest Rh6G film thickness of $0.7 \mathrm{~nm}$, the absorbance spectrum shows two absorbing species, but deconvolution of the emission spectrum revealed that the spectra require three Gaussian curves centered at $535 \pm 3$, $561 \pm 3$, and $592 \pm 3 \mathrm{~nm}$ to obtain satisfactory fits. These peaks are assigned to monomer, excimer, and exciton emission, respectively. When the Rh6G film thickness is increased to $0.9 \mathrm{~nm}$, the only thing that changes is the relative intensities of the previous three peaks. It is not until tRh6G $\geq 2.3 \mathrm{~nm}$ that a fourth Gaussian curve is required, which is located at $644 \pm 3 \mathrm{~nm}$ and is assigned to the emission from larger Rh6G aggregated species.

Table 2. Deconvolution parameters for excitation and emission spectra. Peak position $\left(\lambda_{\max }\right)$ and FWHM $(\Gamma)$ have an uncertainty of $\pm 3 \mathrm{~nm}$.

\begin{tabular}{|c|c|c|c|}
\hline Peak & Excitation $\left(\lambda_{\max } \boldsymbol{\Gamma}\right)(\mathbf{n m})$ & Emission $\left(\lambda_{\max } \boldsymbol{\Gamma}\right)(\mathbf{n m})$ & $\boldsymbol{t}_{\text {Rh6G }}(\mathbf{n m})$ \\
\hline 1 & 495,27 & 535,19 & $0.7-7.0$ \\
\hline 2 & 519,16 & 561,23 & $0.7-7.0$ \\
\hline 3 & N/A & 592,38 & $0.7-7.0$ \\
\hline 4 & N/A & 644,50 & $2.3-7.0$ \\
\hline
\end{tabular}

The reason why the trends in the Rh6G emission differ on the PVDF and glass substrates is presumed to be due to both the surface roughness of the underlying PVDF layer and the lower polarity of the PVDF surface. The smaller surface polarity 
is responsible for the blue-shifted emission peak maxima relative to the glass

substrate, consistent with the absorption spectra. Some of the increased intensity of the emission spectra is due to the increased surface area of the PVDF, because more Rh6G molecules will be available in the area of the excitation area.
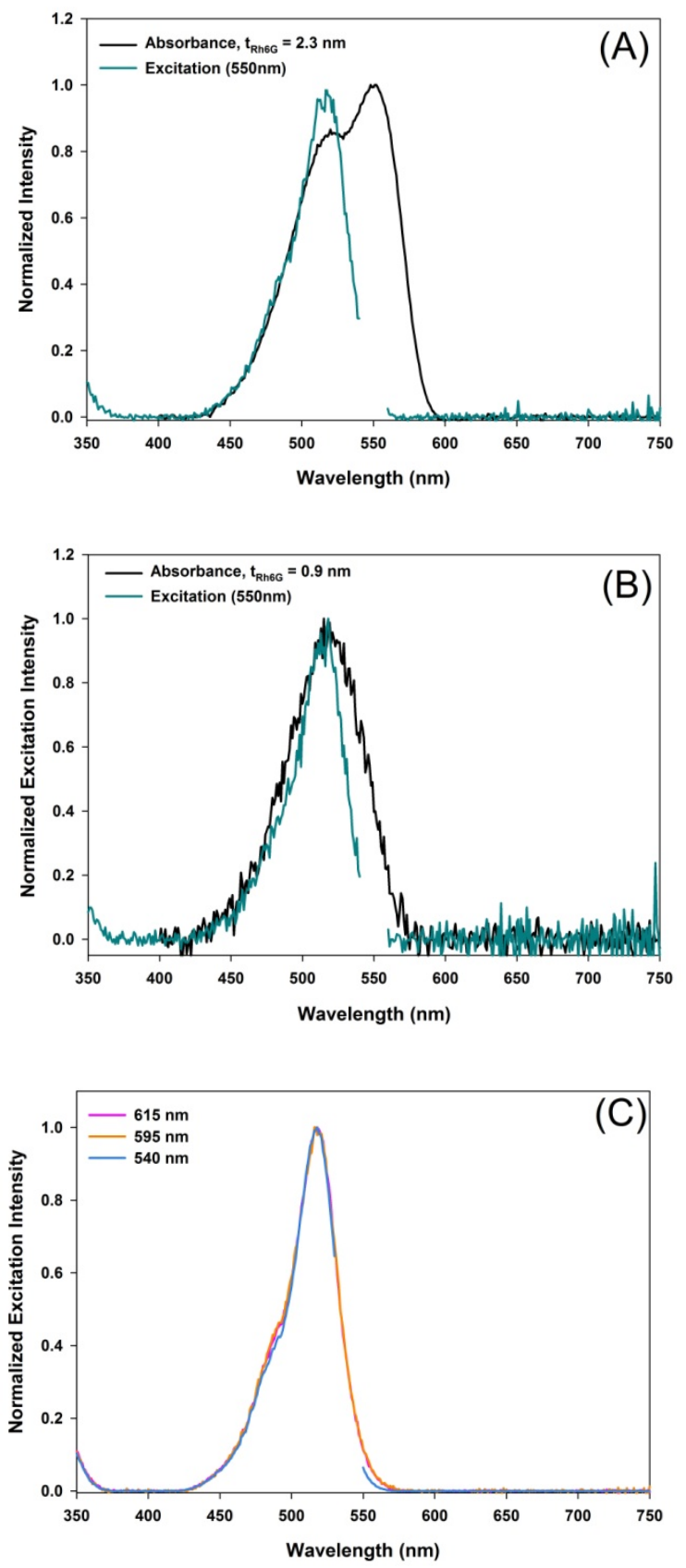
Figure 8. Height normalized absorbance and excitation spectra (detected at $550 \mathrm{~nm}$ ) of Rh6G on PVDF. A) $t_{\text {Rh6G }}=2.3 \mathrm{~nm}$ on $t_{\text {PVDF }}=760 \mathrm{~nm}$. B) $t_{\text {Rh6G }}=0.9 \mathrm{~nm}$ on $t_{\mathrm{PVDF}}=$ $760 \mathrm{~nm}$. C) Excitation spectra of $t_{\mathrm{Rh} 6 \mathrm{G}}=2.3 \mathrm{~nm}$ on $\mathrm{t}_{\mathrm{PVDF}}=760 \mathrm{~nm}$ monitored at different wavelengths.

However, at large thicknesses where aggregates and/or crystallization of Rh6G dominate the surface coverage, the emission of these aggregates is quenched significantly on glass but much less so on PVDF. To help confirm the assignments responsible for the emission, the excitation spectra were measured as shown in Figure 8. The only contribution to the emission is from the species that corresponds to the 518 and $495 \mathrm{~nm}$ absorption peaks, which we have identified as monomer and oblique exciton dimer, respectively. To be sure there is no contribution from the species that absorbs at $560 \mathrm{~nm}$ and to rule out J-type aggregation, the excitation spectra were recorded at different monitoring wavelengths, shown in Figure 8C. At no point in the emission curve does the structure that absorbs at $560 \mathrm{~nm}$ contribute to the emission, and deconvolution of the excitation spectra at any wavelength shows only monomeric and exciton features. The lack of any red-shifted peak in the excitation spectra shows that J-type aggregation is not a contributing factor in the perceived emission enhancement. In Figure 9 can be seen the effect of changing the PVDF film thickness. The intensity of the emission is dependent on the PVDF film thickness but the shape of the spectra is not, which was the same case with the excitation spectra as a function of PVDF thickness as shown in Figure 9C. Because excitation spectra show only the transitions that lead to emission, it makes sense the excitation spectra follow a similar trend. This intensity effect is caused by the way the Rh6G molecules organize more 
randomly on thin PVDF, potentially due to the existence of pinholes exposing the underlying glass substrate or a change in the relative amounts of PVDF phase.
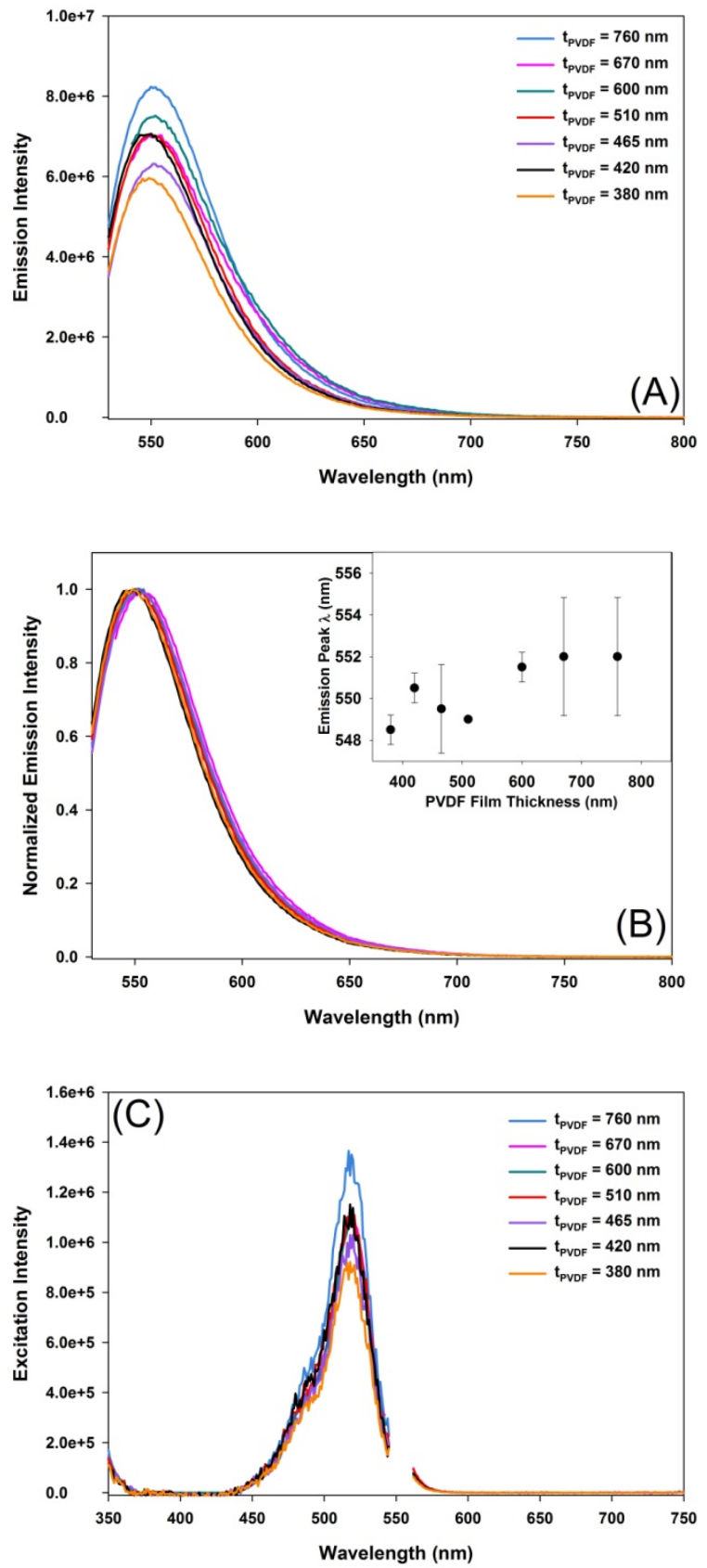


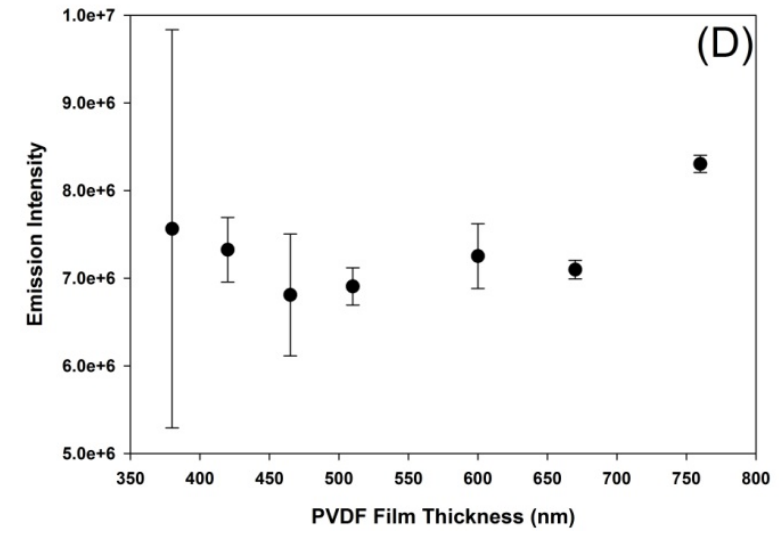

Figure 9. Steady-state fluorescence spectra as a function of PVDF film thickness, $t_{\mathrm{Rh} 6 \mathrm{G}}=2.3 \mathrm{~nm}$. A) Emission spectra as a function of $t_{\mathrm{PVDF}}$. B) Height normalized plot of $\mathrm{A}$, insert shows wavelength dependence on tPVDF. C) Excitation spectra as a function of $t_{\mathrm{PVDF}}$. D) Emission intensity as a function of PVDF film thickness. The error bars represent the standard deviation of two measurements for each thickness.

The normalized plot shows that there is minimal change in the emission maximum when changing the PVDF film thickness. Deconvolution of these emission spectra shows the same peaks as above with just a slight variation in their intensities.

To investigate the changes in the steady-state fluorescence and absorption spectra more in depth, a time-resolved emission spectroscopy (TRES) experiment was performed. Figure 10 shows the decay profiles of different Rh6G thicknesses on a constant thickness of PVDF. At each tRh6G, the decay is multiexponential and strongly dependent on decay collection wavelength. The surface roughness of the PVDF film causes incident light to scatter as well, which has been accounted for in the analysis. As the decays move further away from the source wavelength, the scattering component decreases and the decays are more influenced by the combination of fast and slow fluorescing components. 

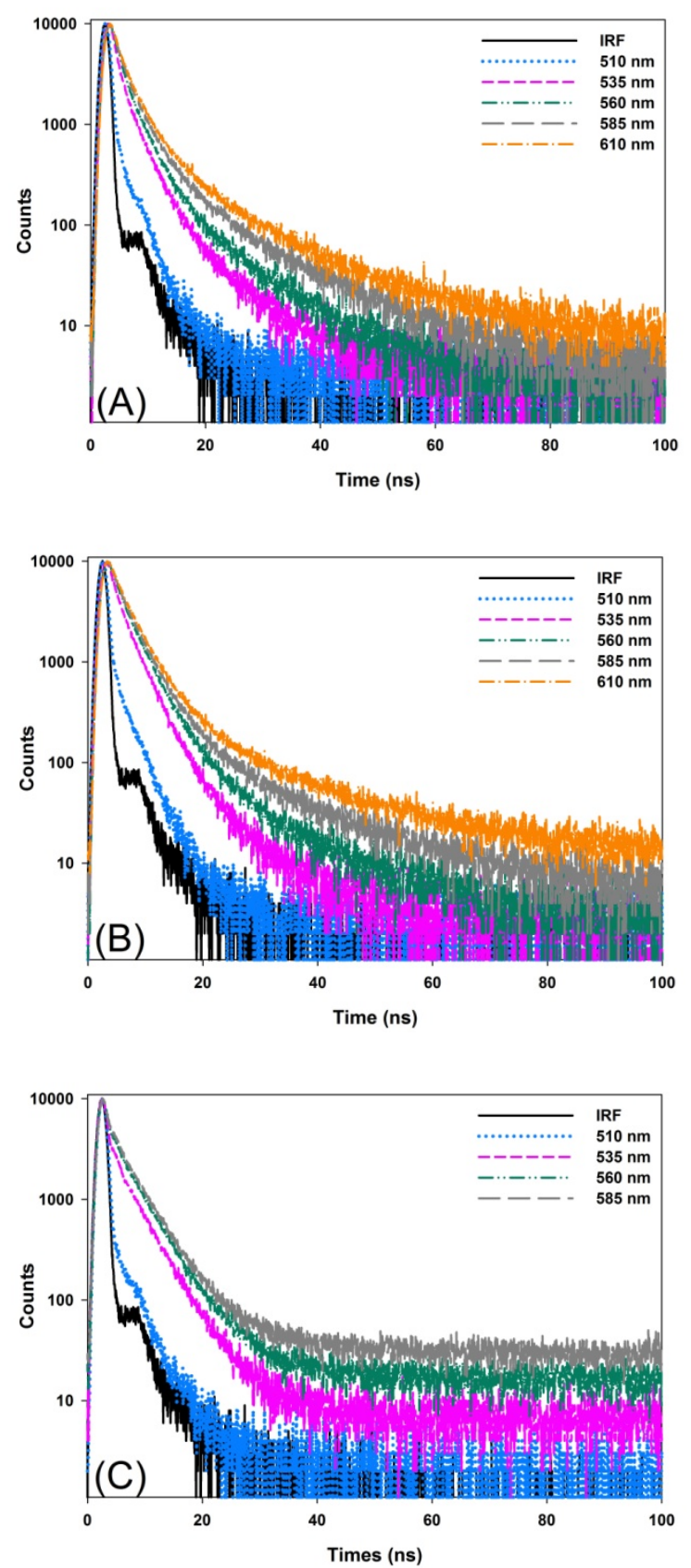

Figure 10. Lifetime decay curves of $t_{\mathrm{Rh} 6 \mathrm{G}}=2.3 \mathrm{~nm}, 0.9 \mathrm{~nm}$, and $0.7 \mathrm{~nm}$ shown in $\mathrm{A}$, $\mathrm{B}$, and $\mathrm{C}$ respectively, $\mathrm{t}_{\mathrm{PVDF}}=760 \mathrm{~nm}$. IRF $=$ instrument response function.

The global multiexponential fitting model used to analyze the wavelength dependent decays was composed of three components for $t_{\mathrm{Rh} 6 \mathrm{G}}=7.0,2.3$, and $0.9 \mathrm{~nm}$, and two 
components for $t_{\mathrm{Rh} 6 \mathrm{G}}=0.7 \mathrm{~nm}$. Because of the roughness of the PVDF film, there is a scattering component present in all models that has been assigned to subnanosecond decay, $\alpha_{1}$, and $\tau_{1}$. The results are given in Table 3 . For $\mathrm{t}_{\mathrm{Rh} 6 \mathrm{G}}=0.7 \mathrm{~nm}$ on PVDF, contrary to the steady-state spectrum deconvolution, the decays show there is only one fluorescing component, and its decay time is $3.9 \pm 0.3$ ns. Because of the lower $\mathrm{S} / \mathrm{N}$ ratio of this measurement and the low surface coverage at this thickness, the excimer and the oblique exciton dimer are not detected. However, the decay time for monomeric Rh6G is consistent with previous reports, ${ }^{35}$ which show that the lifetime of monomeric Rh6G ranges between 3.8 and 4.4 ns in different solvents, with the lower values found in less polar environments. As $t_{\mathrm{Rh} 6 \mathrm{G}}$ increases, the use of a multiexponential function is required to fit the decays, showing that there are two distinct excited states. The shorter lifetime is still assigned to the Rh6G monomer, which decreases as the thickness increases, in agreement with earlier studies showing a similar decrease in lifetime as the concentration of Rh6G increases in aqueous solution. ${ }^{36}$ The decrease in lifetime in aqueous solution was attributed to energy transfer from monomers to nonemissive aggregates, which also likely happens here as there is a sufficient overlap between the emission of monomer Rh6G and the absorbance of aggregated Rh6G as shown in Figure S4. The assignment of the short lifetime to the excited-state monomer is also supported by the TRES. For the $0.7 \mathrm{~nm}$ sample, the spectrum is the same for all times, with a peak maximum at $540 \mathrm{~nm}$. Likewise, the short time spectra for all thicknesses are dominated by the higher energy peak. 


\section{Increasing $\mathrm{t}_{\mathrm{Rh} 6 \mathrm{G}}$}
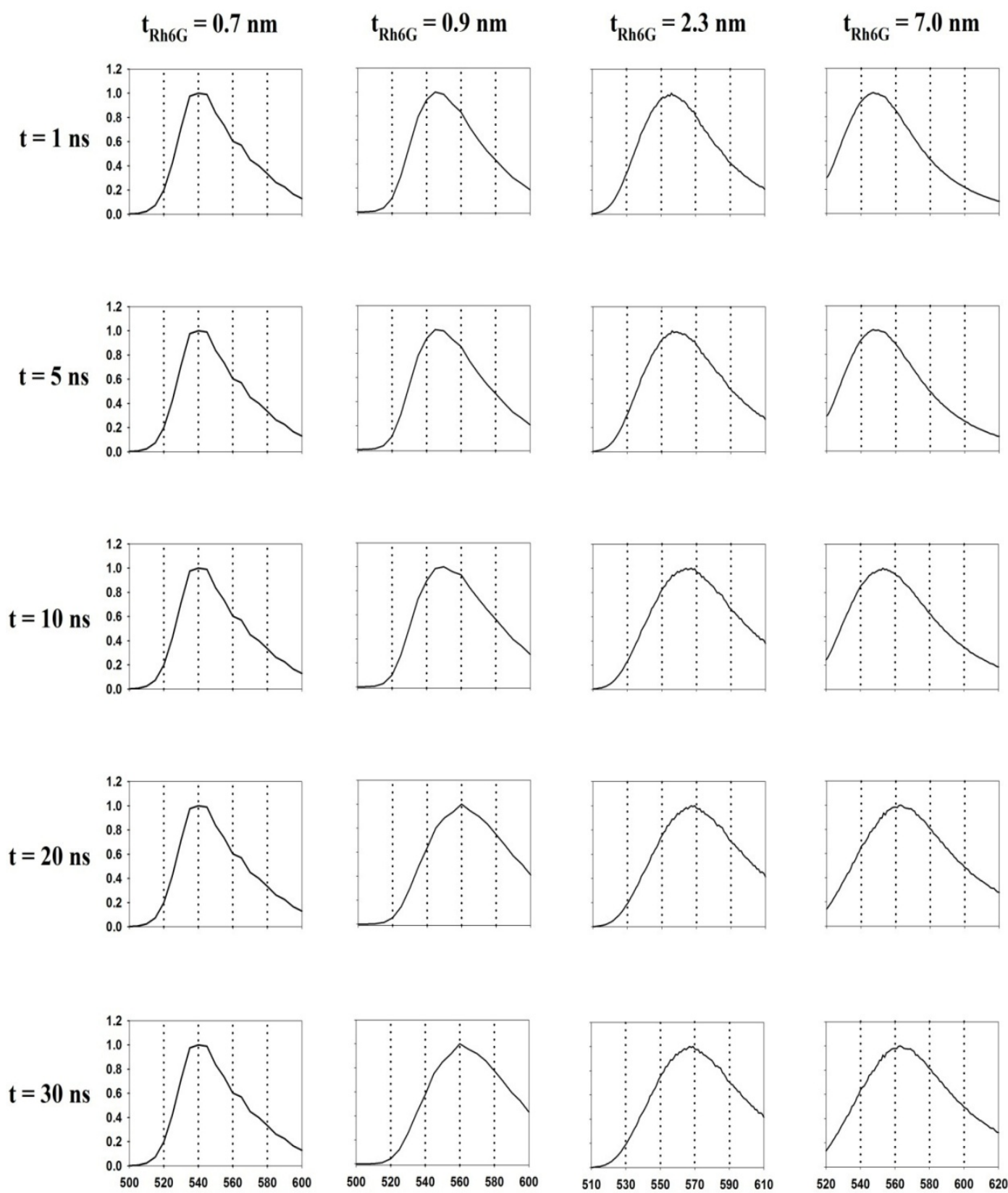

Figure 11. Fluorescence spectra extracted from TRES data at selected times and $t_{\mathrm{Rh} 6 \mathrm{G}}$ with PVDF thickness $=760 \mathrm{~nm}$. 
The longer lifetime excited state is assigned to an excimer because the excitation spectra for this feature arise solely from the monomer absorption. The TRES spectra show that for $\mathrm{tRh} 6 \mathrm{G} \geq 0.9 \mathrm{~nm}$, there is a shift in the emission maximum with respect to time. For the $t_{\mathrm{Rh} 6 \mathrm{G}}=0.9 \mathrm{~nm}$ data in Figure 11, the emission spectra at $\mathrm{t}=1 \mathrm{~ns}$ show a maximum at $\sim 545 \mathrm{~nm}$. As time passes, the dominant emission changes from the fast monomers to the slower excimers, and at $t=20$ ns the emission maximum is at $\sim 560$ $\mathrm{nm}$.

Table 2. Component contribution of the global multi-exponential fit for fluorescence decay curves their associated decay times as a function of $t_{\mathrm{Rh} 6 \mathrm{G}}, \mathrm{t}_{\mathrm{PVDF}}=760 \mathrm{~nm} . \alpha_{\mathrm{i}}$ is a pre-exponential term denoting its relative contribution of the $i^{\text {th }}$ exponential to the overall decay. $\tau_{\mathrm{i}}$ is the time constant for the excited state, or the value of $1 / e$ for the $i^{\text {th }}$ exponential. $\chi^{2}$ is an indication of how good the fit is and should be as close to a value of 1 as possible. The standard deviation in the lifetime is determined from two measurements.

\begin{tabular}{|c|c|c|c|c|c|c|c|}
\hline $\boldsymbol{t}_{\boldsymbol{R h} 6 \mathrm{G}}(\mathrm{nm})$ & $\boldsymbol{\alpha}_{\mathbf{1}}$ & $\boldsymbol{\tau}_{\mathbf{1}}(\mathrm{ns})$ & $\boldsymbol{\alpha}_{\mathbf{2}}$ & $\boldsymbol{\tau}_{\mathbf{2}}(\mathrm{ns})$ & $\boldsymbol{\alpha}_{\mathbf{3}}$ & $\boldsymbol{\tau}_{\mathbf{3}}(\mathrm{ns})$ & $\chi^{2}$ \\
\hline 7.0 & 0.97 & 0.02 & $2.2 \times 10^{-2}$ & $2.2 \pm 0.1$ & $4.9 \times 10^{-4}$ & $9.7 \pm 0.1$ & 1.62 \\
\hline 2.3 & 0.98 & 0.02 & $1.6 \times 10^{-2}$ & $2.4 \pm 0.2$ & $3.9 \times 10^{-4}$ & $10.3 \pm 0.1$ & 1.65 \\
\hline 0.9 & 0.98 & 0.02 & $1.8 \times 10^{-2}$ & $2.9 \pm 0.1$ & $4.7 \times 10^{-4}$ & $11.8 \pm 0.1$ & 1.44 \\
\hline 0.7 & 0.995 & 0.02 & $5.1 \times 10^{-3}$ & $3.9 \pm 0.3$ & N/A & N/A & 1.91 \\
\hline
\end{tabular}

The behavior for $t_{\mathrm{Rh} 6 \mathrm{G}} \geq 2.3 \mathrm{~nm}$ is the same as that for the $0.9 \mathrm{~nm}$ film except the wavelength shift is slightly further to the red $(\sim 570 \mathrm{~nm})$, which can be seen in Figure 11. As thickness increases, the relative ratio of excimer to monomer is increased due to the increased number of neighboring Rh6G molecules. The excimer lifetime also decreases with increasing Rh6G thickness for the same reason as above, energy transfer to nonemissive aggregates. 


\section{Conclusion}

When Rh6G is coated onto a PVDF surface, the emission intensity is significantly higher as compared to a similar coating on a glass surface. Both the absorbance and the emission of the Rh6G species are blue-shifted as compared to the glass surface, which is attributed to changes in surface polarity and the dielectric environment between PVDF and glass. This indicates that the surface of the PVDF film is likely dominated by the nonpolar $\alpha$-phase. The dielectric constant of polymers is highly dependent on the crystallinity of the sample, which has not been measured here, but the fact that no further shifts are observed in both the absorbance and the emission spectra as the PVDF film thickness changes indicates that the dielectric environment and the crystalline phase composition of the PVDF surface are not affected by film thickness in the range studied here. Two factors were determined to contribute to the perceived emission enhancement. First, the PVDF layer can act as a light trap where the incident light is internally reflected between the Rh6G/PVDF and PVDF/glass interfaces. This effect leads to more photons being absorbed by the Rh6G layer, which can translate to a higher probability of emission, and a more efficient use of the incident light. Second, the surface roughness of the PVDF film strongly influences the photophysics of the system. The rough surface provides a larger total true surface area for an excitation beam to interact with, thereby exciting more molecules. Further, as indicated by the absorbance spectra, when changing only the film thickness of the Rh6G layer, the populations of monomer and aggregated Rh6G grow concurrently instead of gradually shifting from one species to the other as is 
observed on a smooth glass substrate. The strongly emissive monomeric form of Rh6G remains at a higher relative concentration as the Rh6G film becomes nominally thicker. Through the use of TRES, it was found that the emission comes from a combination of monomers and excimers. However, once the Rh6G film thickness surpasses $0.9 \mathrm{~nm}$, the emission intensity starts to plateau. The decrease in the excitedstate lifetime for both the monomer and the excimer with respect to increasing Rh6G film thickness is attributed to energy transfer to large aggregates. This also accounts for the plateau in the emission intensity for the thicker Rh6G films. Although the data presented herein support the fact that surface roughness plays a critical role in the photophysics of Rh6G thin films, additional systems similar to that studied are necessary to distinguish whether the effect is purely physical or if there are chemical effects as well, which is the subject of future work.

\section{ASSOCIATED CONTENT}

\section{Supporting Information}

The Supporting Information is available free of charge on the ACS Publications website at DOI: 10.1021/acs.langmuir.7b00144. Absorption spectra with fits, emission spectra with fits, and excitation spectra with fits.

\section{AUTHOR INFORMATION}

\section{Corresponding Author}

*E-mail: weuler@chm.uri.edu.

ORCID William B. Euler: 0000-0002-5317-8510 


\section{Notes}

The authors declare no competing financial interest.

\section{ACKNOWLEDGEMENT}

This material is based upon work supported by the U.S. Department of Homeland Security, Science and Technology Directorate, Office of University Programs, under Grant Award 2013-ST-061-ED0001. The views and conclusions contained in this document are those of the authors and should not be interpreted as necessarily representing the official policies, either expressed or implied, of the U.S. Department of Homeland Security.

\section{REFERENCES}

1. Lakowitz, J. R. Principles of Fluorescence Spectroscopy, $3^{\text {rd }}$ ed.; Springer: New York, 2006, 237-242.

2. Staahlberg, J; Almgren, M. Polarity of Chemically Modified Silica Surfaces and Its Dependence on Mobile-Phase Composition by Fluorescence Spectrometry. Anal. Chem., 1985, 57, 817 - 821.

3. Sackett, D.L.; Wolff, J. Nile Red as a Polarity-Sensitive Fluorescent Probe of Hydrophobic Protein Surfaces. Anal Biochem., 1987, 167, 228 - 234.

4. Chang, K; Forcé, R. K. Time-Resolved Pyrene Fluorescence for Determination of Polymer Surface Polarity: Correlations with Surface Tension. Appl. Spectr., 1995, 49, 211 - 215.

5. Hayashi, Y.; Kawada, Y.; Ichimura, K. Dicyanoanthracene as a Fluorescence 
Probe for Studies on Silica Surfaces. Langmuir, 1995, 11, 2077 - 2082.

6. Sunahara, H.; Urano, Y.; Kojima, H; Nagano, T. Design and Synthesis of a Library of BODIPY-Based Environmental Polarity Sensors Utilizing Photoinduced Electron-Transfer-Controlled Fluorescence ON/OFF Switching. J. Am. Chem. Soc., 2007, 129, 5597 - 5604.

7. Baldi, L. D. C.; Iamazaki, E. T.; Atvars, T. D. Z. Evaluation of the Polarity of Polyamide Surfaces Using the Fluorescence Emission of Pyrene. Dyes and Pigments, 2008, 76, $669-676$.

8. Habenicht, S. H.; Schramm, S.; Fischer, S.; Sachse, T.; Herrmann-Westendorf, F.; Bellmann, A.; Dietzek, B.; Presselt, M.; Weiß, D.; Beckert, R.; Görlse, H. Tuning the Polarity and Surface Activity of Hydroxythiazoles - Extending the Applicability of Highly Fluorescent Self-Assembling Chromophores to SupraMolecular Photonic Structures. J. Mater. Chem. C, 2016, 4, 958 - 971.

9. McCrate, J. M.; Ekerdt, J. G. Coverage-Dependent Luminescence from TwoDimensional Systems of Covalently Attached Perylene Fluorophores on Silica. J. Phys. Chem. C, 2014, 118, $2104-2114$.

10. Chapman, M.; Mullen, M.; Novoa-Ortega, E.; Alhasani, M.; Elman, J. F.; Euler, W. B. Structural Evolution of Ultrathin Films of Rhodamine 6G on Glass. J. Phys. Chem. C, 2016, 120, 8289 - 8297.

11. Jelley, E. E. Spectral Absorption and Fluorescence of Dyes in the Molecular State. Nature, 1936, 1009 - 1010.

12. Kasha, M. Energy Transfer Mechanisms and the Molecular Exciton Model for Molecular Aggregates. Radiat. Res., 1963, 20, 55 - 71.

13. Arbeloa, F. L.; Gonzalez, I. L.; Ojeda, P. R.; Arbeloa, I. L. Aggregate Formation of Rhodamine 6G in Aqueous Solution. J. Chem. Soc. Faraday Trans.2, 1982, 78, $989-994$.

14. Valdes-Aguilera, O.; Neckers, D. C. Aggregation Phenomena in Xanthene Dyes. Acc. Chem. Res., 1989, 22, 171 - 177. 
15. Ojeda, P. R.; Amashta, I. A. K.; Ochoa, J. R.; Arbeloa, I. L. Excitonic Treatment and Bonding of Aggregates of Rhodamine 6G in Ethanol. J. Chem. Soc. Faraday Trans.2, 1988, 84, $1-8$.

16. Kemnitz, K.; Yoshihara, K. Entropy-Driven Dimerization of Xanthene Dyes in Nonpolar Solution and Temperature-Dependent Fluorescence Decay of Dimers. J. Phys. Chem., 1991, 95, 6095 - 6104.

17. Elking, M. D.; He, G.; Xu, Z. Molecular Orientation of Submonolayer Rhodamine-6G on Quartz Substrates: A Comparative Study Using Reflection and Transmission UV-Vis Spectroscopy. J. Chem. Phys., 1996, 105, 6565 6573.

18. Sasai, R.; Fujita, T.; Iyi, N.; Itoh, H.; Takagi, K. Aggregated Structures of Rhodamine 6G Intercalated in a Fluor-Taeniolite Thin Film. Langmuir, 2002, $18,6578-6583$.

19. Martinez, V. M.; Arbeloa, F. L.; Prieto, J. B., Lopez, T. A.; Arbeloa, I. L. Characterization of Rhodamine 6G Aggregates Intercalated in Solid Thin Films of Laponite Clay. 1. Absorption Spectroscopy. J Phys. Chem. B, 2004, 108, 20030 - 20037.

20. Shundo, A.; Okada, Y.; Ito, F.; Tanaka, K. Fluorescence Behavior of Dyes in Thin Films of Various Polymers. Macromolecules, 2011, 45, 329 - 335.

21. Soffer, B.H.; Linn, J.W. Continuously Tunable Picosecond-Pulse Organic-Dye Laser. J. Appl. Phys., 1968, 39, 5859 - 5860.

22. Encinas, M. V.; Rufs, A. M.; Bertolotti, S. G.; Previtali, C. M.Xanthene Dyes/Amine as Photoinitiators of Radical Polymerization: A Comparative and Photochemical Study in Aqueous Medium. Polymer, 2009, 50, 2762 - 2767.

23. Zhang, Z.; Zheng, Y.; Hang, W.; Yan, X.; Zhao, Y. Sensitive and Selective Off-On Rhodamine Hydrazide Fluorescent Chemosensor for Hypochlorous Acid Detection and Bioimaging. Talanta, 2011, 85, 779 - 786. 
24. Latendresse, C. A.; Fernandes, S.C.; You, S.; Zhang, H. Q.; Euler, W.B. A Fluorometric Sensing Array for the Detection of Military Explosives and IED Materials. Anal. Methods, 2013, 5, 5457 - 5463.

25. Zhang, H. Q.; Euler, W. B. Detection of Gas-Phase Explosive Analytes Using Fluorescent Spectroscopy of Thin Films of Xanthene Dyes. Sens. Actuators B, 2016, 225, $553-562$.

26. Matoian, M.A.; Sweetman, R.; Hall, E.C.; Albanese, S.; Euler, W.B. Light Trapping to Amplify Metal Enhanced Fluorescence with Application for Sensing TNT. J. Fluoresc., 2013, 23, 877 - 880.

27. Benz, M.; Euler, W. B.; Gregory, O. J. The Influence of Preparation Conditions on the Surface Morphology of Poly(vinylidene fluoride) Films. Langmuir, 2001, 17, 239-243.

28. Benz, M.; Euler, W. B.; Gregory, O. J. The Role of Solution Phase Water on the Deposition of Thin Films of Poly(vinylidene fluoride). Macromolecules, 2002, 35, $2682-2688$.

29. Benz, M.; Euler, W.B. Determination of the Crystalline Phases of Poly(vinylidene fluoride) Under Different Preparation Conditions using Differential Scanning Calorimetry and Infrared Spectroscopy. J. Appl. Polym. Sci., 2003, 89, $1093-1100$.

30. Branciforti, M. C.; Sencadas, V.; Lanceros-Mendez, S.; Gregorio Jr, R. New Technique of Processing Highly Oriented Poly(vinylidene fluoride) Films Exclusively in the $\beta$ Phase. J. Polymer Sci. Part B, 2007, 45, 2793 - 2801.

31. Yingxue, X.; Huiqing, F.; Weiguo, L.; Chen, Y.; Xiaoling, N.A Study on Thermo-optic Effect of $\beta$ Poly(vinylidene fluoride) Thin Films Prepared by Solution Casting Method. Surf. Rev. Lett., 2008, 15, 175 - 181.

32. Martins, P.; Nunes, J. S.; Hungerford, G.; Miranda, D.; Ferreira, A.; Sencadas, V.; Lanceros-Mendez, S. Local Variation of the Dielectric Properties of Poly(vinylidene fluoride) During the $\alpha$ - to $\beta$-Phase Transformation. Phys. Lett. A, 2009, 373, 177 - 180. 
33. Sencadas, V.; Lanceros-Mendez, S.; Gregorio Jr, R. $\alpha$ to $\beta$ Phase Transformation and Microstructural Changes of PVDF Films Induced by Uniaxial Stretch. J. Macromol. Sci. B: Phys., 2009, 48, 514 - 525.

34. Vijayakumar, R. P.; Khakhar, D. V.; Misra, A. Studies on $\alpha$ to $\beta$ Phase Transformations in Mechanically Deformed PVDF Films. J. Appl. Polym. Sci., 2010, 117, 3491 - 3497.

35. Magde, D.; Wong, R.; Seybold, P. G. Fluorescence Quantum Yields and Their Relation to Lifetimes of Rhodamine 6G and Fluorescein in Nine Solvents: Improved Absolute Standards for Quantum Yields. Photochem.Photobiol., 2002, 75, 327-334.

36. Fedoseeva, M.; Letrun, R.; Vauthey, E. Excited-State Dynamics of Rhodamine 6G in Aqueous Solution and at the Dodecane/Water Interface. J. Phys. Chem. B, 2014, 118, 5184-5193.

37. Kobayashi, M.; Tashiro, K.; Tadokoro, H. Molecular Vibrations of Three Crystal Forms of Poly(vinylidene fluoride). Macromolecules, 1975, 8, 158 171.

38. Bachmann, M.A.; Gordon, W.L.; Koenig, J.L.; Lando, J.B. An Infrared Study of Phase-III Poly(vinylidene fluoride). J. Appl. Phys., 1979, 50, 6106 - 6112.

39. Tashiro, K.; Kobayashi, M.; Tadokoro, H. Vibrational Spectra and DisorderOrder Transition of Poly(vinylidene fluoride) Form-III. Macromolecules, 1981, 14, $1757-1764$.

40. Milani, A.; Castiglioni, C.; Radice, S. Joint Experimental and Computational Investigation of the Structural and Spectroscopic Properties of Poly(vinylidene fluoride) Polymorphs. J. Phys. Chem. B, 2015, 119, 4888 - 4897.

41. Griffiths, D. J. Introduction to Electrodynamics, $3^{\text {rd }}$ ed. Prentice-Hall: Upper Saddle River, NJ, 2007. 
The Influence of Interfacial Effects on the Photophysics of Rhodamine 6G Thin Films on a Poly(vinylidene fluoride) Surface

\author{
Matthew Mullen and William B. Euler* \\ Department of Chemistry \\ 140 Flagg Road \\ Kingston, RI 02881
}

\title{
Supporting Information
}

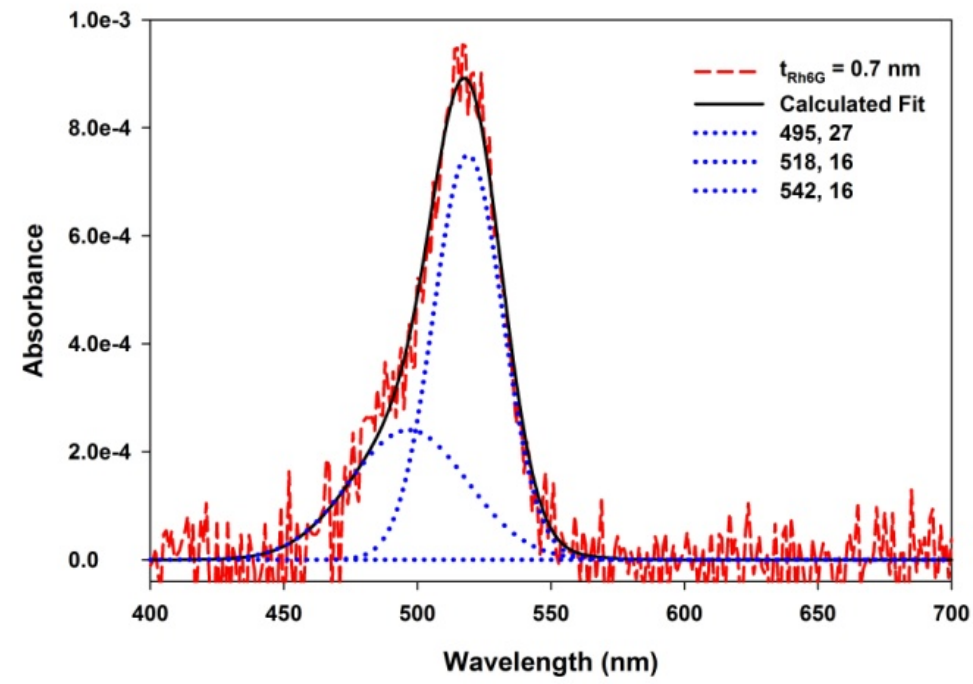



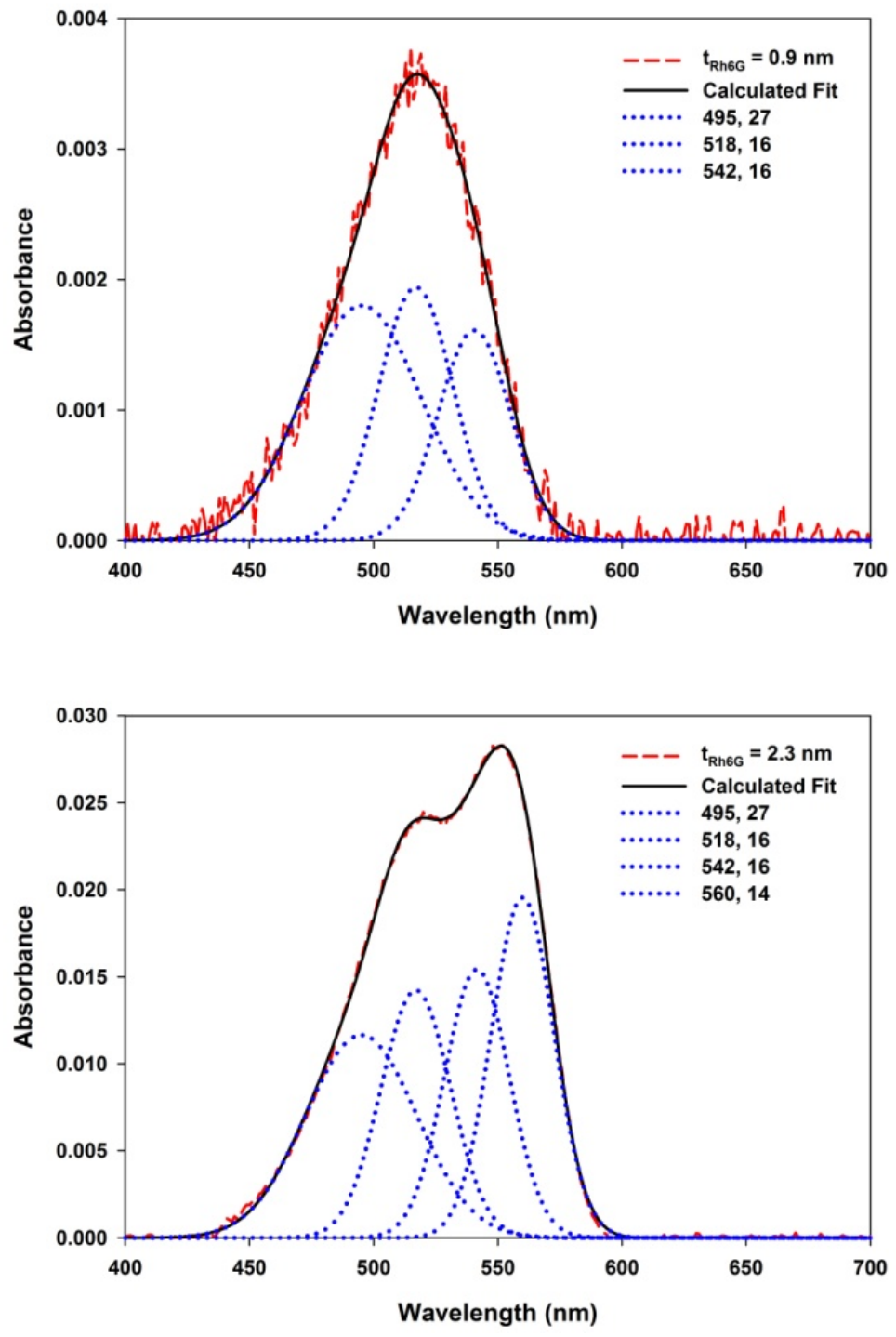


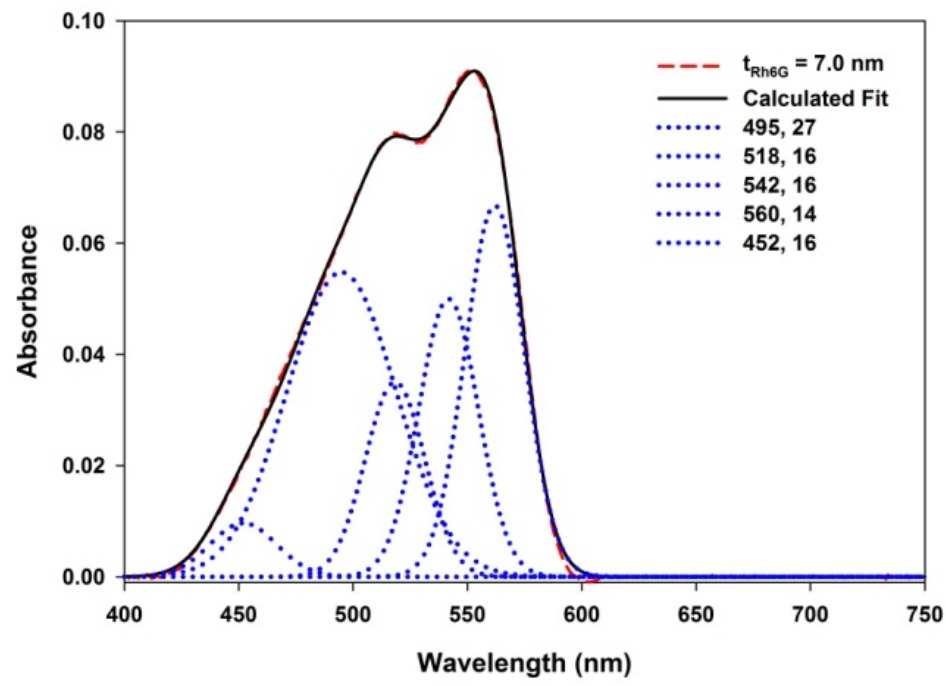

Figure S1. Fit lines constructed from the deconvolution of the absorbance spectra as a function of $t_{\text {Rh6G }}$. $t_{P V D F}=760 \mathrm{~nm}$. Legend shows peak position and full-width-at-halfmaximum in $\mathrm{nm}$.

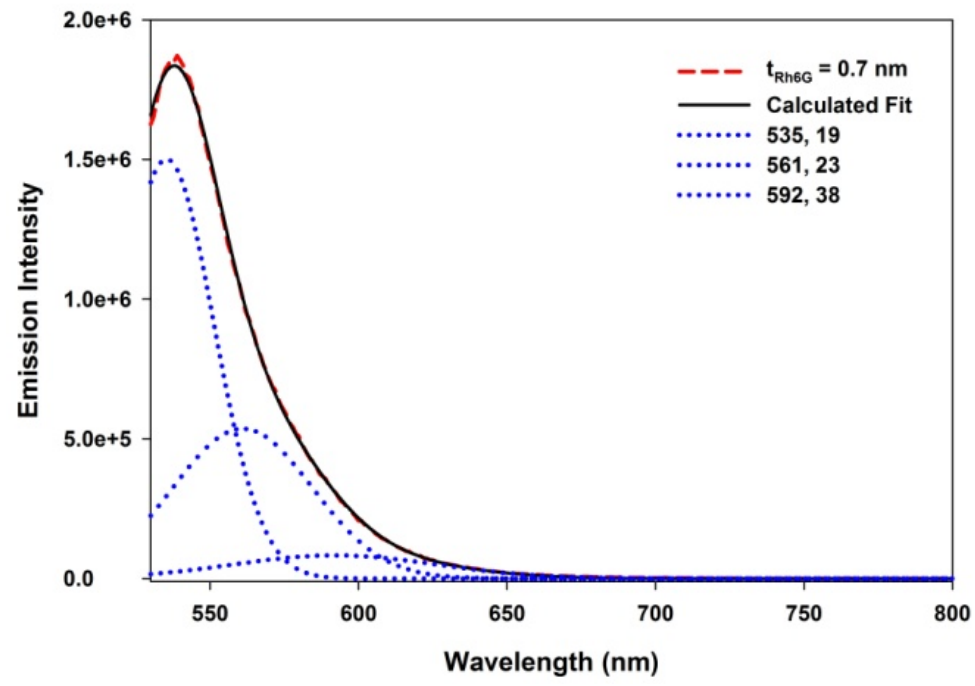



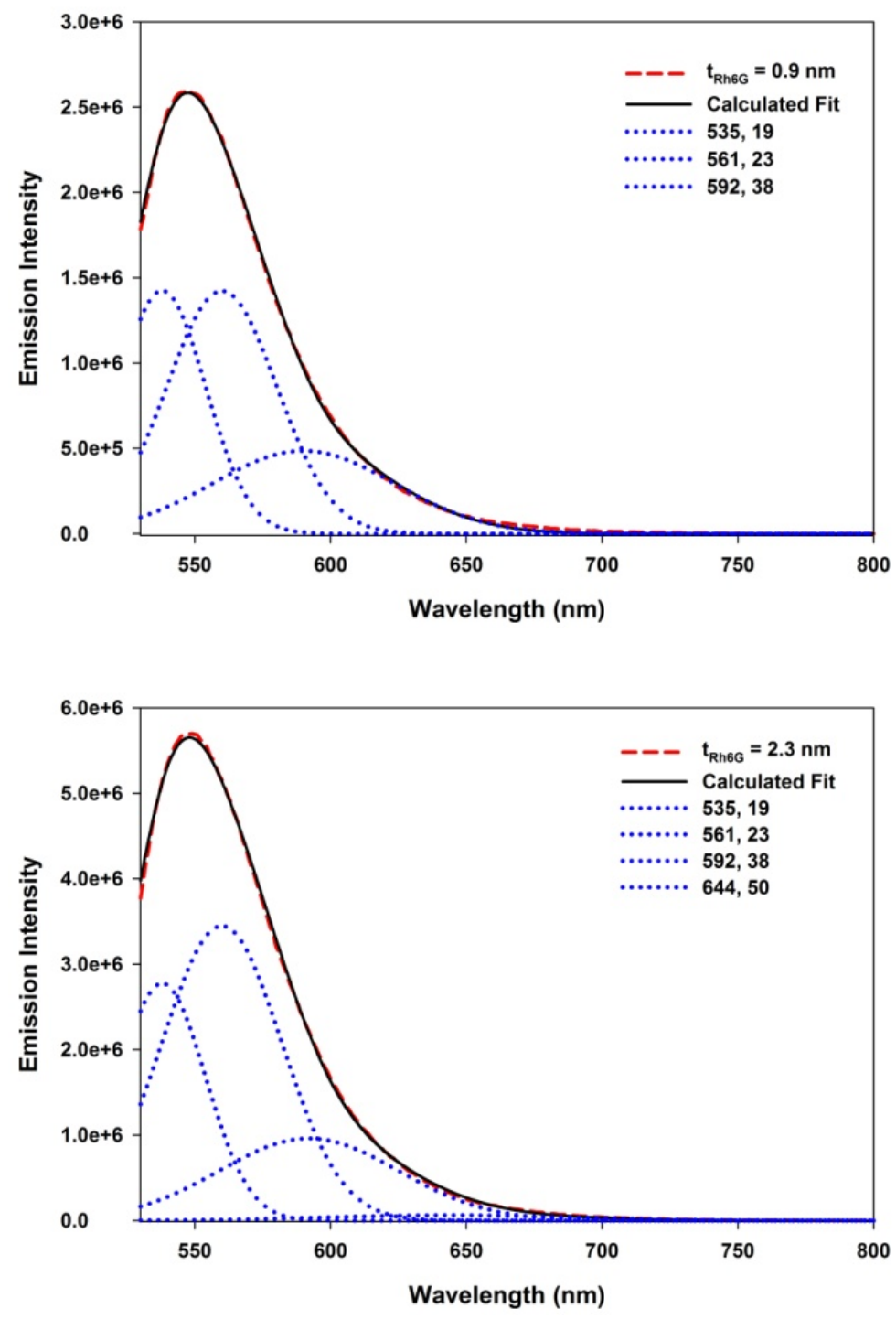


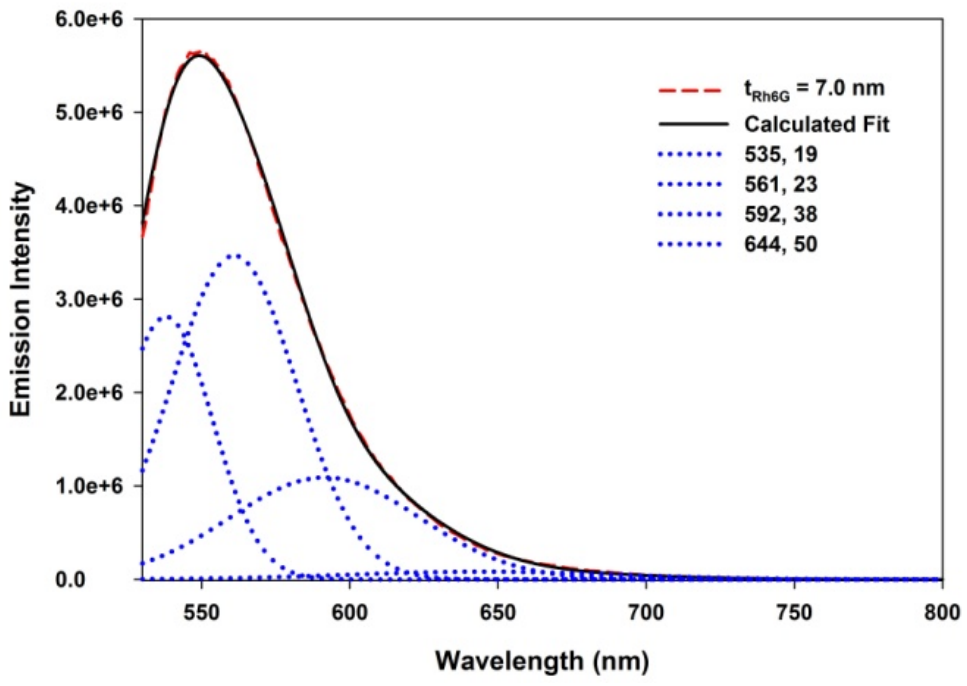

Figure S2. Fit lines constructed from the deconvolution of the emission spectra as a function of $t_{\mathrm{Rh} G \mathrm{G}}$. $t_{\mathrm{PVDF}}=760 \mathrm{~nm}$. Legend shows peak position and full-width-at-halfmaximumin $\mathrm{nm}$. 

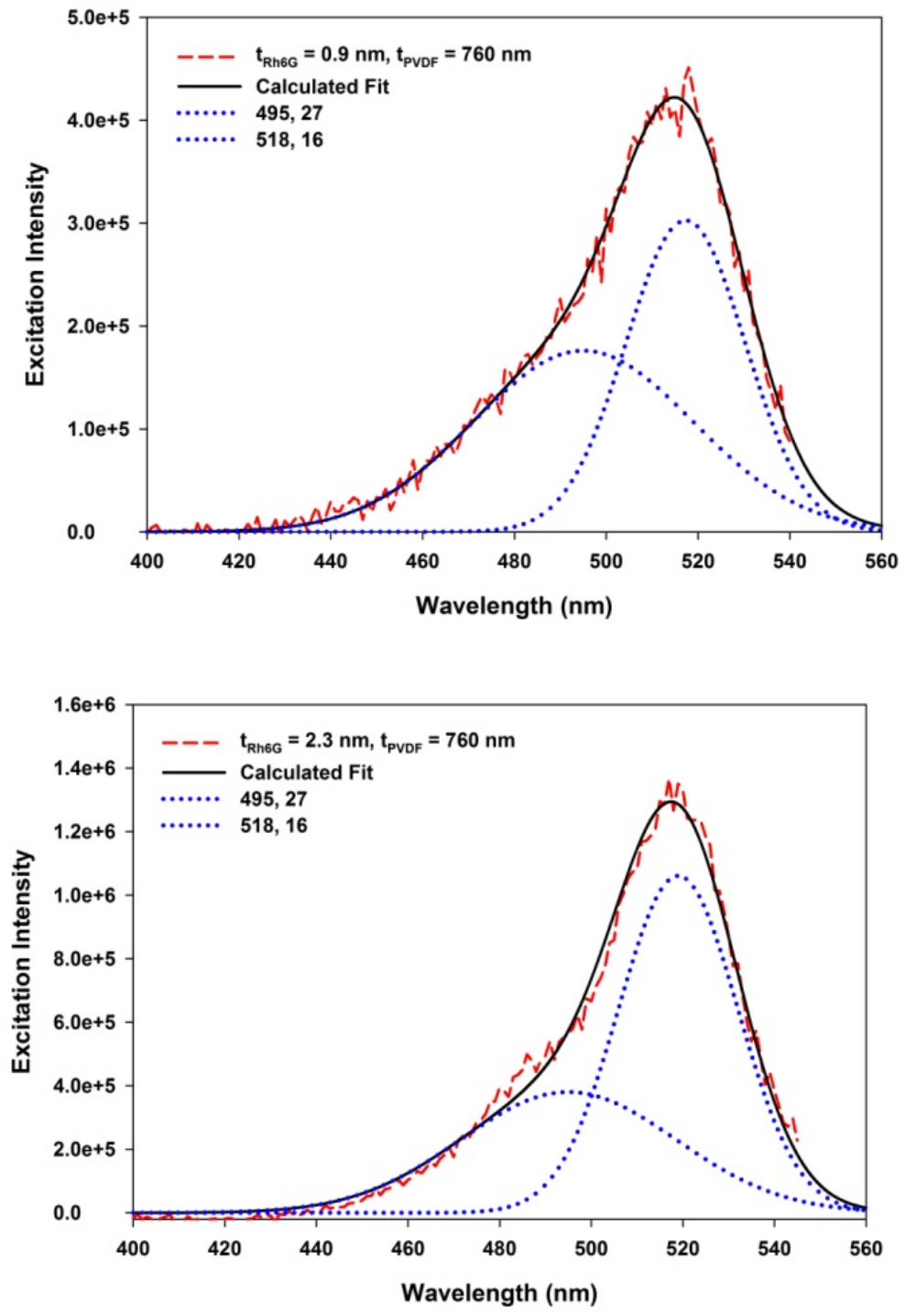


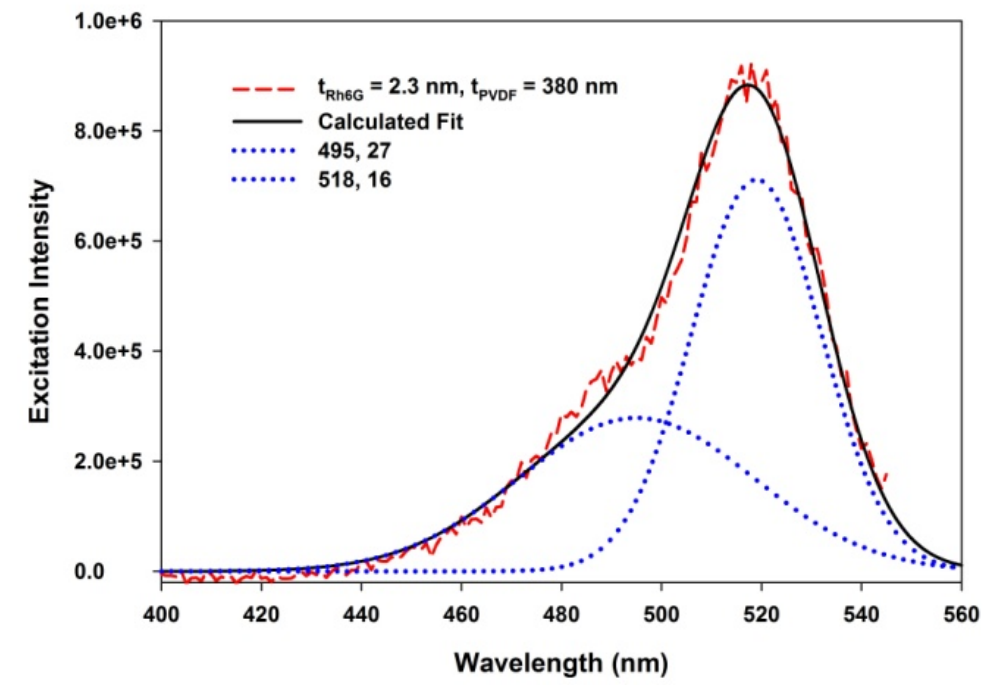

Figure S3. Fit lines constructed from the deconvolution of the excitation spectra of various combination of $t_{\mathrm{PVDF}}$ and $\mathrm{t}_{\mathrm{Rh} 6 \mathrm{G}}$. Legend shows peak position and full-width-athalf-maximumin $\mathrm{nm}$.

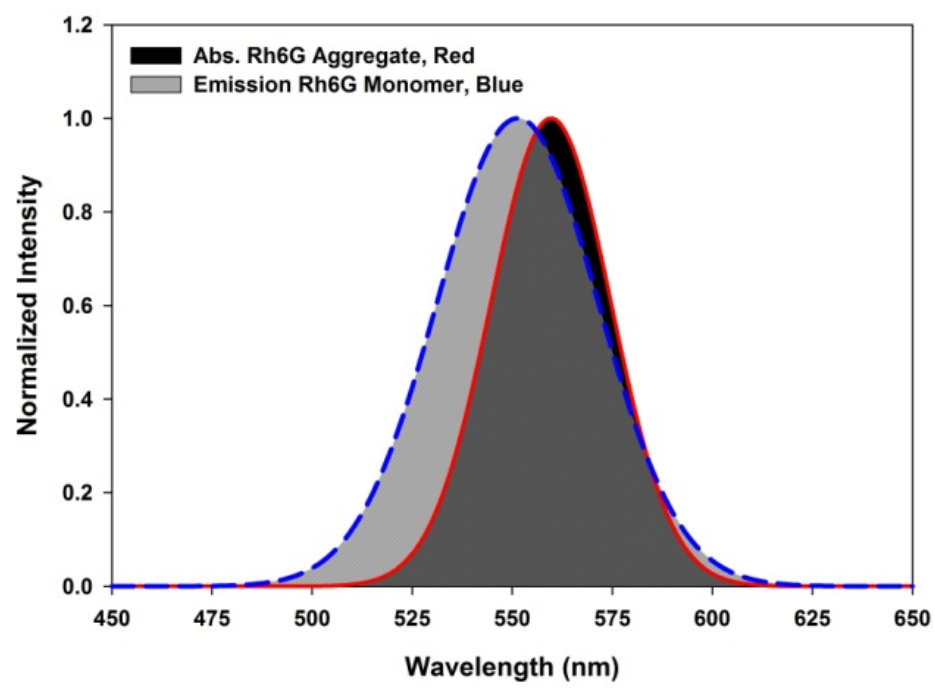

Figure S4. Spectral overlap of the deconvoluted peaks from the emission of monomeric Rh6G (light gray area, dashed blue line) with the absorbance of aggregated Rh6G (black area, solid red line). 


\title{
CHAPTER 2
}

\section{Surface Morphology Dependence on Molecular Weight in Polystyrene Thin Films: Formation of Periodic Structures by Spin-Casting}

\author{
Matthew Mullen and William B. Euler* \\ Department of Chemistry \\ 140 Flagg Road \\ Kingston, RI 02881
}

The following is in preparation to be submitted to Macromolecules and is presented here in manuscript format 


\begin{abstract}
The surfaces of various molecular weight polystyrene thin films have been studied using optical profilometry. The thin films are fabricated through the use of spin casting and the surface displays two different types of structures depending on the location on the film. Pillars are formed in the center of the sample where the solution is deposited and wrinkles are formed at the extremities, extending out from the center. The wrinkle characteristics, such as wavelength (in the $\mu \mathrm{m}$ range) and amplitude (in the nm range), can be tuned through the molecular weight used to cast the film or by modulating the solvent evaporation rate for a given molecular weight.
\end{abstract}

\title{
1. Introduction
}

In the present state of materials science, where there is a push for devices to be made smaller, it is important to understand a material's physical and chemical properties from both the perspective of the surface as well as the bulk. It is well documented that a material can behave differently when comparing these two regions of the same substance, ${ }^{1-5}$ which is mostly attributed to confinement effects. Furthermore, an additional area becomes of importance when the surfaces of two different materials interact to form an interface. In devices comprised of layered thin films this interfacial region can have a large effect on the overall behavior of the device and therefore brings about the importance to understand the interactions that can occur at the interface. Understanding and controlling the chemical and physical interfacial properties of a layered thin film system would allow for more control over 
the devices behavior and may be used in the fabrication of such devices to tailor them for a specific purpose.

A defining feature of an interface in a layered thin film system is the surface morphology of the underlying layer. Surface morphology is a broad term and can be considered on many different scales. In modern thin film systems one must consider the nano/micrometer regions of surface morphology as well as the macro features. Changing and controlling surface morphology through the use of etching and lithography in hard materials such as silicon wafers has been well studied and understood for some time now, ${ }^{6-8}$ however with polymers playing a larger role in devices new methods are needed to control surface morphology. The push for understanding and controlling surface morphologies arises mostly from the field of adhesion, ${ }^{9,10}$ but certain patterning in materials has been shown to be promising for use in photonic devices ${ }^{11-17}$ and stretchable microelectronics. ${ }^{18,19}$ Methods currently used for producing surface patterns in soft materials include thin film dewetting, ${ }^{20-22}$ electrohydrodynamic patterning, ${ }^{23,24}$ breath figures, ${ }^{25,26}$ thermal-gradient induced patterning, ${ }^{27,28}$ self assembly and microphase separation, ${ }^{22,29,30}$ but most commonly used is template based patterning. ${ }^{31-33}$ Template based patterning can be used to create various different types of geometric structures such as hexagons, stripes, or rectangles, however the most common use of templating is the production of wrinkles on a surface. $^{34-37}$ To induce wrinkling on a surface, a layer of elastomer, typically polydimethylsiloxane (PDMS), is prestrained mechanically or thermally. A rigid skin layer is then transferred onto the elastomer and the strain is released, which causes buckling of the skin layer and produces wrinkles, creases, or folds. 
Herein we report a simple spin casting method that can be used to fabricate polystyrene thin films that have different surface morphologies based primarily on the molecular weight of the polystyrene (PS) used in the casting solution. Not only does the wrinkling in the PS films form spontaneously without the use of PDMS, but two different types of structures are formed. Pillars form in the center of the sample where the solution is deposited that transition into wrinkles away from the center. Higher $\mathrm{M}_{\mathrm{w}}$ PS films require a kinetic component to be considered, however by changing the rate of spinning this adds another layer of control over the surface morphology.

\section{Experimental Section}

Glass microscope slides were cut into $3.75 \mathrm{~cm} \mathrm{x} 1.75 \mathrm{~cm}$ pieces and cleaned by sonication in 95\% ethanol (EtOH, Pharmaco-Aaper) for 15 min followed by another round of sonication in DI water for 15 min. The substrates are then dried with $\mathrm{N}_{2}$. Polystyrene (PS, Polymer Source Inc.) solutions of different molecular weights (PDI < 1.1) were made in tetrahydrafuran (THF, Sigma Aldrich, HPLC Grade) and sonicated for 1 hour. The solution concentration is represented by a weight to volume ratio. Once all the PS is in solution, the PS films are made by spin casting. The glass substrate was placed on the stage and a $500 \mu \mathrm{L}$ aliquot of PS solution was deposited in the center. The substrate was spun for $45 \mathrm{~s}$ at room temperature with an acceleration of $1080 \mathrm{~s}^{-2}$. To achieve different PS film thickness either the spin speed or the PS concentration was varied. After spinning, the films were dried in a $60^{\circ} \mathrm{C}$ oven for 1 $\min$. 
The film thickness of the PS was determined using a Filmetrics F40 microscope via reflection spectroscopy. The reflection spectrum was recorded with a tungsten-halogen light source over the range of 400-900 $\mathrm{nm}$. The resulting interference pattern was then fit with a calculated spectrum of given thickness and refractive index. After the film thickness was determined, the surface morphology of the PS films was analyzed using the Filmetrics Profilm 3D Optical Profiler. The images of the surface are obtained vertical scanning interferometry (VSI) and phase shifting interferometry (PSI).

\section{Results and Discussion}

The spin coating method for fabricating thin films is often used because of its ability to create uniform films of a desired thickness with good reproducibility. However, the actual dynamics of spin casting are complicated, as the final product and quality of the film depend on many factors. As Scriven et al ${ }^{38}$ have demonstrated, the final thickness of a dry film can be estimated through the following equation,

$$
h=\chi_{p o l}^{o}\left[\eta_{o}\left(\frac{c D_{g}}{v_{g}{ }^{1 / 2} \rho^{2}}\right)\left(\frac{p_{\text {sol }} M_{\text {pol }}}{R T}\right)\left(1-\chi_{p o l}^{o}-\chi_{\text {sol }}^{\infty}\right)\right]^{1 / 3} \frac{1}{\omega^{1 / 2}}
$$

where $\eta_{o}$ is the initial viscosity of the solution, $\chi_{\text {pol }}^{o}$ is the mass fraction of polymer in the initial solution, $\chi_{\text {sol }}^{\infty}$ is the mass fraction of the pure solvent in the gas phase at equilibrium, $v_{g}$ is the kinematic viscosity of the overlying gas, $D_{g}$ is the diffusivity of the overlying gas, $\rho$ is the density of the solution, $p_{s o l}$ is the vapor pressure of the pure solvent, $M_{\text {pol }}$ is the molecular weight of the polymer, $R$ and $T$ represent the ideal gas constant and temperature, and $\omega$ is the angular speed. Equation [1] demonstrates that changing the molecular weight of the polymer will have an effect on the thickness of the film that is cast both explicitly through the $M_{\text {pol }}$ 
term and implicitly through the initial viscosity, since $\eta_{o} \propto M_{\text {pol }}^{n}$, where n depends on how the polymer chains coil. Figure 1 shows the determined PS film thickness as a function of PS molecular weight, while all other variables are kept constant.

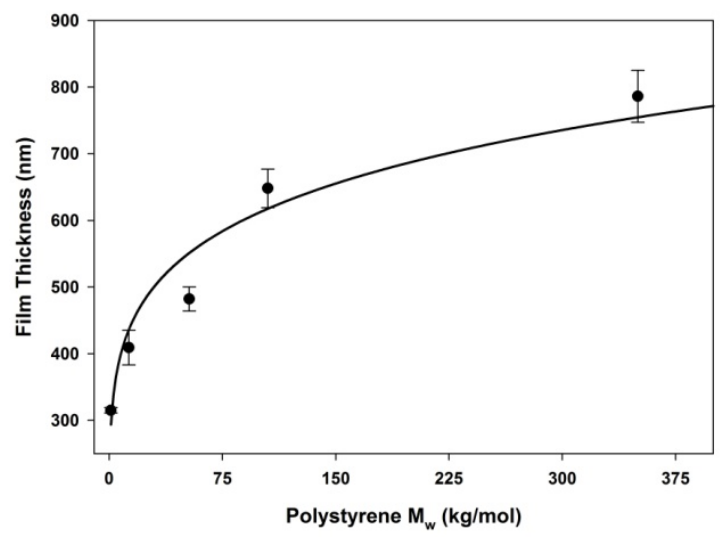

Figure 1. Film thickness as a function of PS $\mathrm{M}_{\mathrm{w}}$. The data are fit to the equation $\mathrm{h}=\mathrm{ax} \mathrm{x}^{\mathrm{b}}$, where $b=1 / 6$. Thin films are made from a 4\% PS solution and spun at a fixed speed of 1200 RPM. The error bars represent a standard deviation of 9 measurements.

As predicted by equation [1], the film thickness scales to a power of $1 / 6$ with respect to the molecular weight of the polystyrene. In order to make a proper comparison between the films of different PS molecular weight, it is important to ensure they are all of the same thickness, as material properties in thin film systems have been observed with changes in film thickness. $^{39-42}$ Therefore, in order to achieve a uniform film thickness for all samples, the parameters of the spin coating process were analyzed. First, equation [1] implies that the desired average film thickness could be achieved by changing the spin speed. Figure 2 shows the relationship between average PS film thickness and spin speed for $\mathrm{M}_{\mathrm{w}}=350000 \mathrm{~g} / \mathrm{mol}$. 


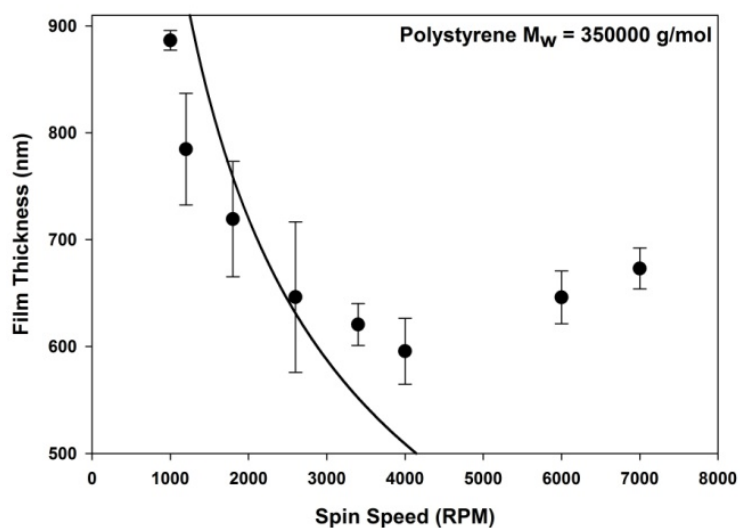

Figure 2. Polystyrene average film thickness as a function of spin speed for $\mathrm{M}_{\mathrm{w}}=350000$ $\mathrm{g} / \mathrm{mol}$. The solid line represents a fit of $\mathrm{h}=\mathrm{ax} \mathrm{x}^{\mathrm{b}}$, where $\mathrm{b}=-1 / 2$. The error bars represent $\mathrm{a}$ standard deviation of 4 measurements.

The film thickness and spin speed are inversely related, but as the fit in figure 2 shows, equation [1] is not sufficient to describe film thickness as a function of angular speed. Equation [1] trends down for $\omega>4000$ RPM, however there is a divergence from the fit when $\omega>2600$ RPM, which indicates additional forces acting on the film that are not considered in equation [1]. Therefore, the effect of the initial concentration of the PS casting solutions on the film thickness was analyzed and is shown in figure 3.

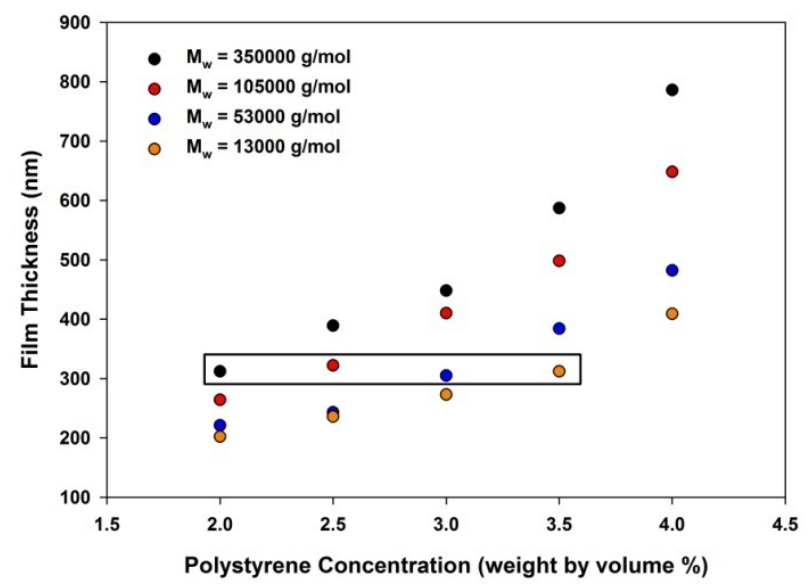

Figure 3. Film thickness as a function of PS concentration spun at 1200 RPM for multiple molecular weights. The box indicates the thickness region of the $M_{w}=1000 \mathrm{~g} / \mathrm{mol} \mathrm{film}$. 
As expected, the film thickness decreases substantially with decreasing PS concentration. For $\mathrm{M}_{\mathrm{w}}=350000,105000,53000$, and $13000 \mathrm{~g} / \mathrm{mol}$ the PS concentration was made to be $2.0 \%$, 2.5\%, 3.0\%, and 3.5\%, respectively. With these initial concentrations, the resulting film thickness was in the region of $300 \mathrm{~nm}$ and the surface morphologies of all $\mathrm{M}_{\mathrm{w}}$ 's could now be compared to each other.

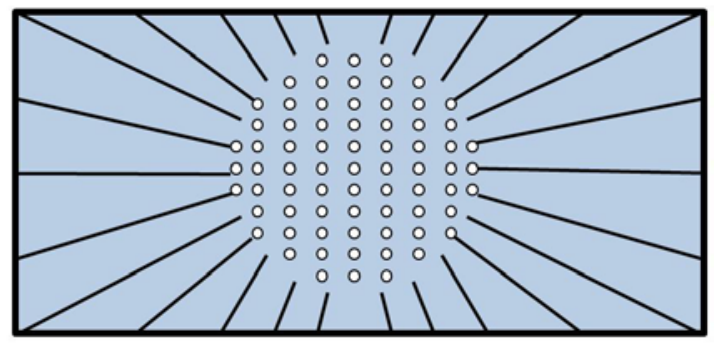

- Pillar

- Wrinkle

Figure 4. Graphic displaying the location of the different types of structures on the PS sample.
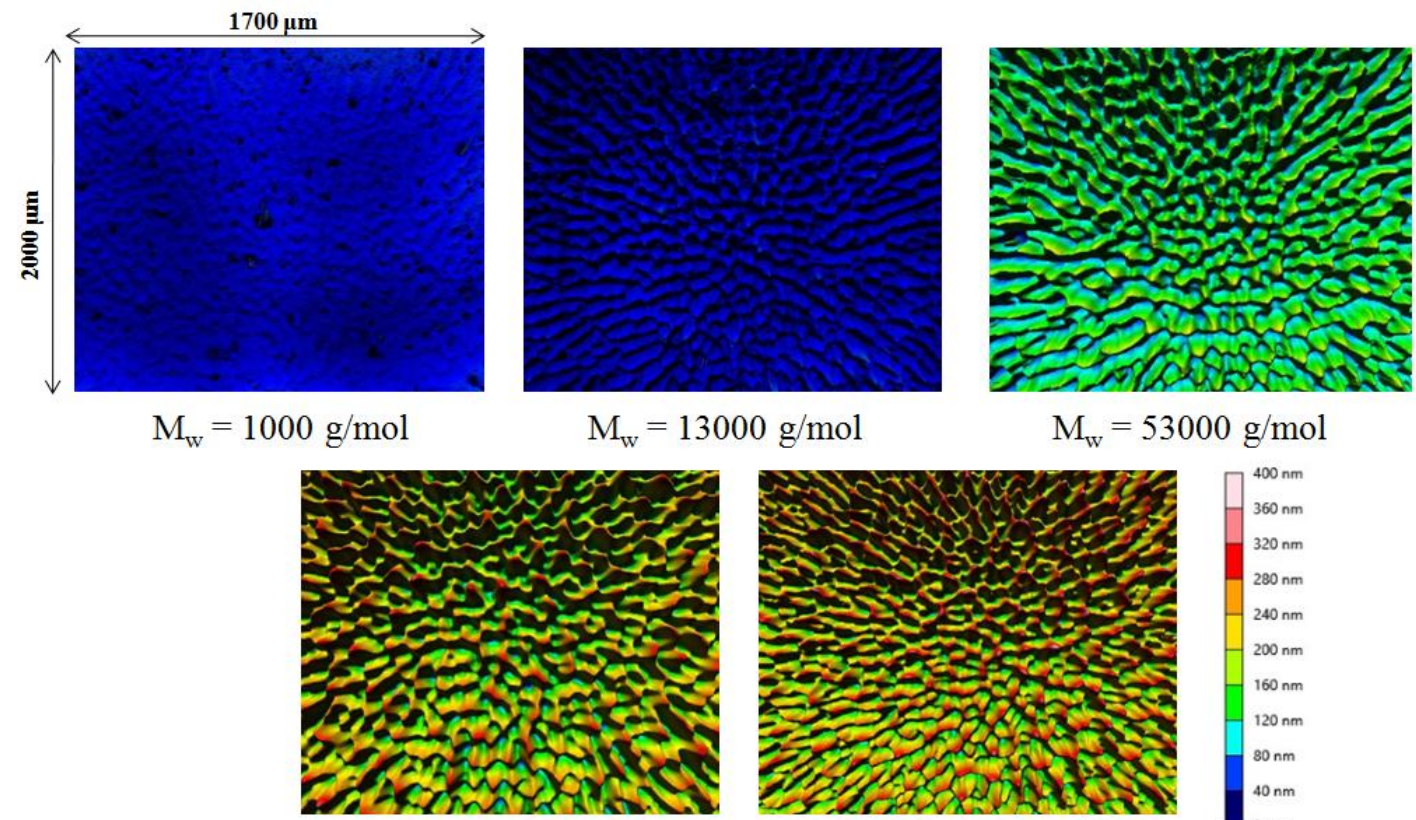

$$
\mathrm{M}_{\mathrm{w}}=105000 \mathrm{~g} / \mathrm{mol}
$$

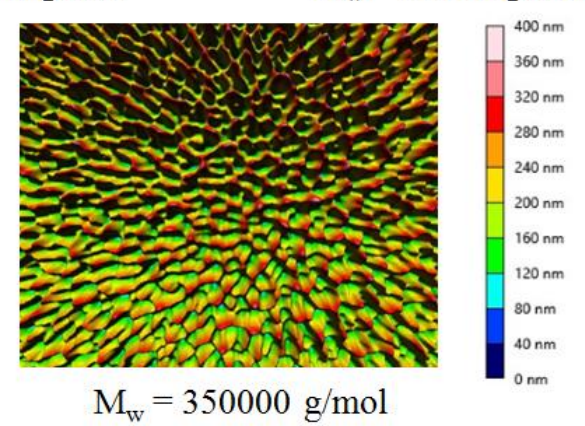

Figure 5. Top down view of select PS films of different molecular weight. Images are $2 \mathrm{~mm} x$ $1.7 \mathrm{~mm}$ and collected at the center of the sample. Color represents differences in height of features. All films are $310 \pm 6$ nm thick. 

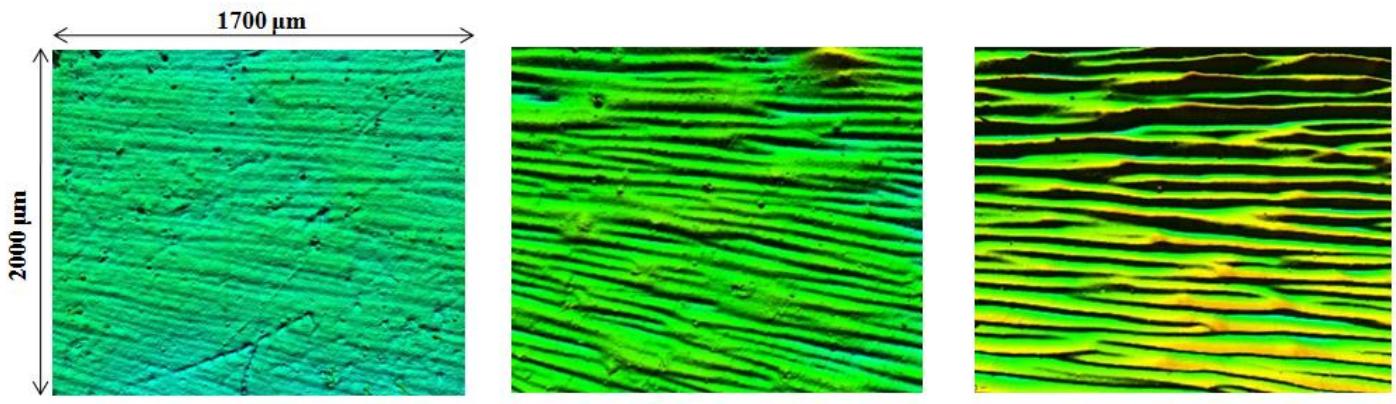

$\mathrm{M}_{\mathrm{w}}=1000 \mathrm{~g} / \mathrm{mol}$

$$
\mathrm{M}_{\mathrm{w}}=13000 \mathrm{~g} / \mathrm{mol}
$$

$\mathrm{M}_{\mathrm{w}}=53000 \mathrm{~g} / \mathrm{mol}$

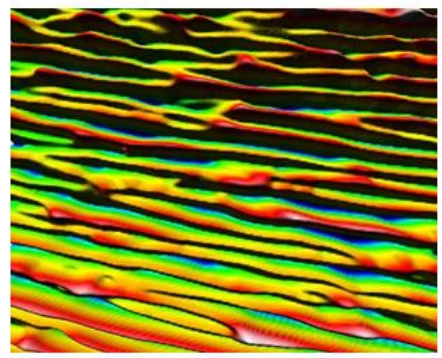

$\mathrm{M}_{\mathrm{w}}=105000 \mathrm{~g} / \mathrm{mol}$

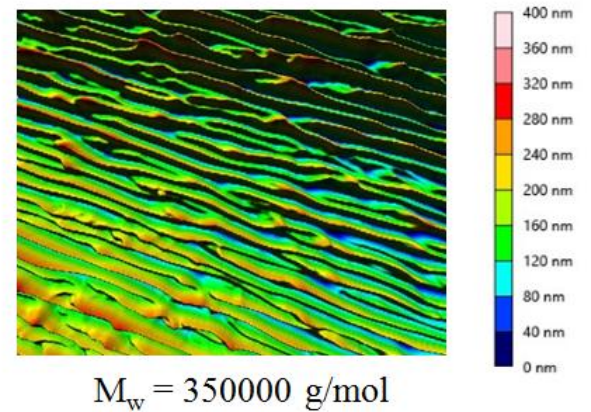

$\mathrm{M}_{\mathrm{w}}=350000 \mathrm{~g} / \mathrm{mol}$

Figure 6. Top down view of select PS films of different molecular weight. Images are $2 \mathrm{~mm} x$ $1.7 \mathrm{~mm}$ and collected on one side of the sample. Color represents differences in height of the features. All films are $310 \pm 6 \mathrm{~nm}$ thick.

Optical profilometry was used to determine the surface morphology of the various $\mathrm{M}_{\mathrm{w}}$ PS films. Figures 5 and 6 show how the surface morphology of the PS films changes as the molecular weight of the PS changes. These structures form spontaneously upon drying of the films with no external stress applied to the samples. First thing to note is the difference in the surface features of the two imaged areas. As the images in figure 5 show, the center of the film forms micron sized pillars at the base with varying heights on the nanometer scale. Figures 7A and 7B show how the characteristics of the pillars changes as a function of PS $M_{w}$. At PS $M_{w}$ $<53 \mathrm{~kg} / \mathrm{mol}$, the pillars are not well defined with heights are $<20 \mathrm{~nm}$ and wavelengths of $70-$ $85 \mu \mathrm{m}$. However, changing the $\mathrm{M}_{\mathrm{w}}$ of the PS used to cast the film has a drastic effect on the characteristics of the pillars. When PS $\mathrm{M}_{\mathrm{w}}$ is in the range of $53 \mathrm{~kg} / \mathrm{mol} \leq \mathrm{M}_{\mathrm{w}} \leq 105 \mathrm{~kg} / \mathrm{mol}$, the pillars become more defined. The heights increase to 50 - $120 \mathrm{~nm}$ and the wavelengths 
increase to $90-115 \mu \mathrm{m}$. On the contrary, when PS $\mathrm{M}_{\mathrm{w}}=350 \mathrm{~kg} / \mathrm{mol}$ both the height of the pillars and the wavelength decrease to the range of $\sim 80 \mathrm{~nm}$ and $70 \mu \mathrm{m}$, respectively.

Looking at the images in figure 6, it is clear that there is a big change in the surface morphology as the point of view moves away from the center and off to one side of the films. The pillars at the center transition into wrinkles that extend out from the center like spokes on a wheel, which is shown diagrammatically in figure 4. Like the pillars, the wrinkle amplitude and wavelength are dependent on the $\mathrm{M}_{\mathrm{w}}$ of PS used to cast the film. The wrinkles have similar characteristics to the pillars in the sense that the wavelengths are on the micron scale and the amplitudes are on the nanometer scale. Figures 7C and 7D show how these characteristics change as a function of PS $M_{w}$. Like the pillar structures, when $M_{w}$ is low the features are not well defined, having amplitudes of $<20 \mathrm{~nm}$ and wavelengths in the range of $60-80 \mu \mathrm{m}$. When $\mathrm{M}_{\mathrm{w}}$ is increased to the range of $105 \mathrm{~kg} / \mathrm{mol} \geq \mathrm{M}_{\mathrm{w}} \geq 53 \mathrm{~kg} / \mathrm{mol}$, both the amplitude and wavelength grow as well to values of $20-140 \mathrm{~nm}$ and $100-115 \mu \mathrm{m}$, respectively. Finally, when $\mathrm{M}_{\mathrm{w}}=350 \mathrm{~kg} / \mathrm{mol}$ the amplitude decreases to $70 \mathrm{~nm}$ and the wavelength to $\sim 70 \mu \mathrm{m}$, in agreement with the behavior of the pillars.

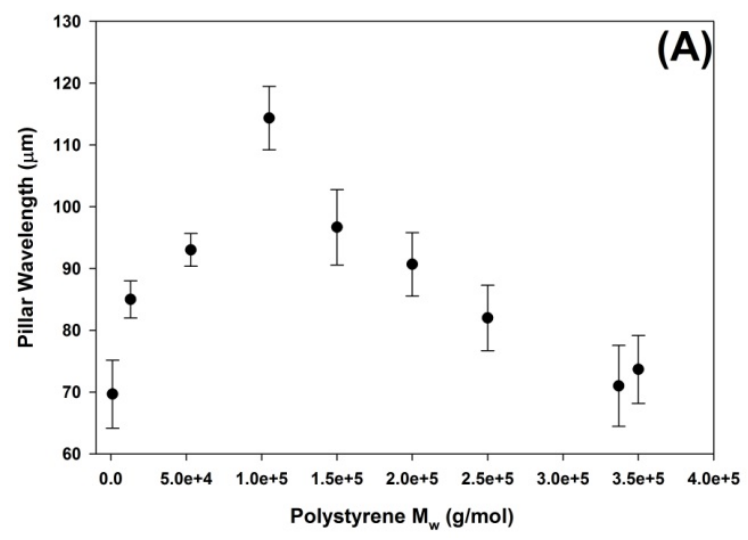



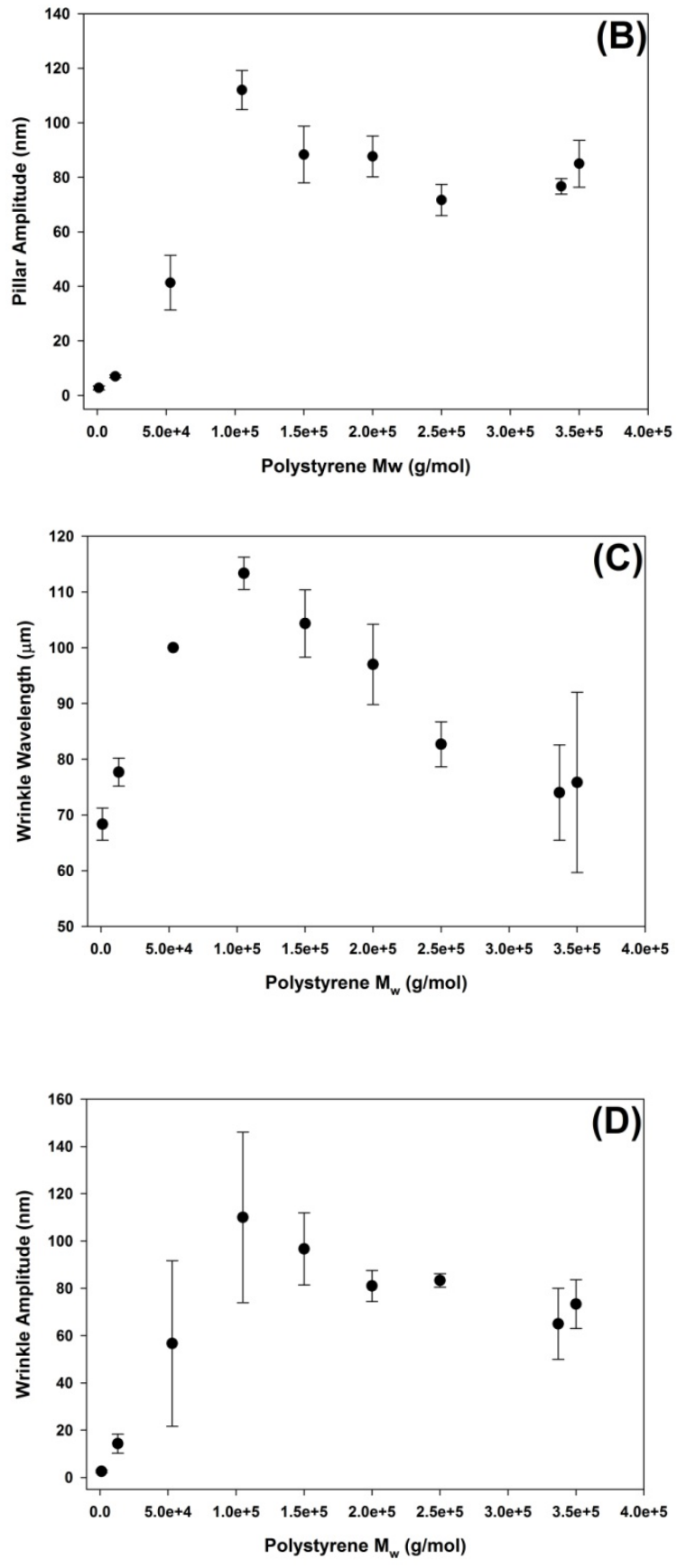

Figure 7. Average A) Pillar wavelength, B) Pillar amplitude, C) Wrinkle Wavelength, and D) Wrinkle Amplitude as a function of polystyrene molecular weight. Error bars represent a standard deviation of three measurements. 
The wrinkle wavelength and amplitude were determined by fitting a sine wave of the form,

$$
h=h_{o}+a_{o} \sin \left(\frac{2 \pi x}{\lambda_{o}}+b\right)
$$

where $h_{o}$ is the mean film thickness if the zero of the y-axis is set at the top of the substrate, $a_{0}$ and $\lambda_{0}$ are the amplitude and wavelength of the waveform, and $b$ is an arbitrary phase term dependent on where $\mathrm{x}=0$ is set. The fits for both the pillars and the wavelengths can be seen in the supporting information figures S1and S2. Both the wrinkle amplitude and wavelength increase with molecular weight until some critical value is surpassed and they start to decrease, as evidenced by the $350 \mathrm{~kg} / \mathrm{mol}$ values in figure 7.

Wrinkling in homogenous films on rigid substrates has been discussed ${ }^{43,44}$ and is mostly attributed to expansion from swelling of the polymer or from drying from a swollen state. However, this method usually leads to unstable wrinkle patterns that eventually give way to creases and folds instead of wrinkles. M. Ramanathan et.al. ${ }^{45}$ have described spontaneous wrinkling in poly(2-vinyl-4,4-dimethylaxlactone) (PVDMA) thin films similar to what is reported herein, but do not make mention of the film thickness regime they worked in. Although film thickness was not studied they did conclude that the wrinkling observed in the PVDMA films was caused solely by the film and the substrate the film was supported on had no effect on the wrinkling wavelength or amplitude.

In the present system being discussed here, the film is in a completely swollen state as it is deposited onto the substrate and dried throughout the spinning process. In a study done by Allain and Pauchard ${ }^{46}$ on polysaccharide drops, they observed surface instabilities as the polymer drops dried. The buckling in the dried state of the drops is attributed to a glassy layer forming at the air/liquid interface which then experiences stress as the liquid like layer underneath decreases in volume as evaporation proceeds. The combination of these two previous studies suggests that a similar phenomenon is happening in the PS films, where a 
more rigid, glassy surface layer is produced as the film is being formed. As the underlying liquid like layer decreases in volume from solvent evaporation, buckling occurs in the glassy layer and causes wrinkle formation. This process is described through a schematic in figure 8.
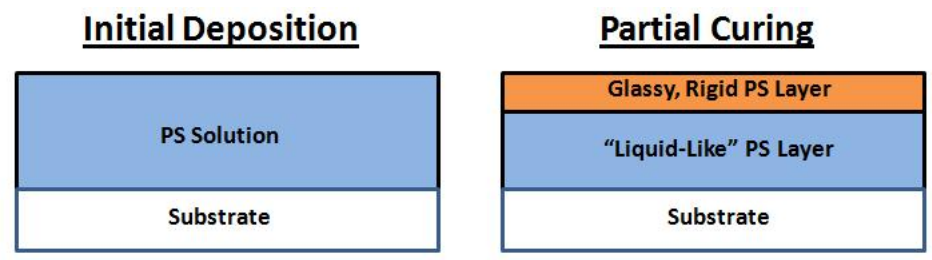

Complete Curing

Substrate
PS Thin Film

Figure 8. 2D Schematic showing how the drying process leads to wrinkle formation in the PS thin films.

Wrinkle wavelength can be predicted through use of the formula ${ }^{43}$,

$$
\lambda=2 \pi t_{s}\left(\frac{E_{s}}{3 E_{b}}\right)^{1 / 3}
$$

where $\lambda$ is the wrinkle wavelength, $t_{s}$ is the skin thickness, and $E_{s}$ and $E_{b}$ are the elastic moduli of the skin film and bulk substrate. Equation 3 evolves from studies where wrinkle patterns are formed through the use of bilayers, i.e. transferring a rigid skin film to an elastic polydimethylsiloxane (PDMS) substrate, however with the model presented here for the PS films, this equation should be sufficient to describe the wrinkles. The skin layer is the rigid PS surface and the bulk is the liquid-like layer that is still swollen with solvent. The difficulty that arises from using eq. 3 to describe the wrinkles in this system is that the skin thickness is unknown and the elastic moduli of the different layers of PS are difficult to consider without direct measurement. With that being said, it is implied that the wrinkling only occurs in the skin layer, or some portion of it, and therefore the amplitude of the wrinkles should be proportional to the skin thickness. Additionally, because the skin is formed by a glassy PS layer at the surface the glass transition temperature, $T_{g}$, must also be considered as a function of PS molecular weight. 


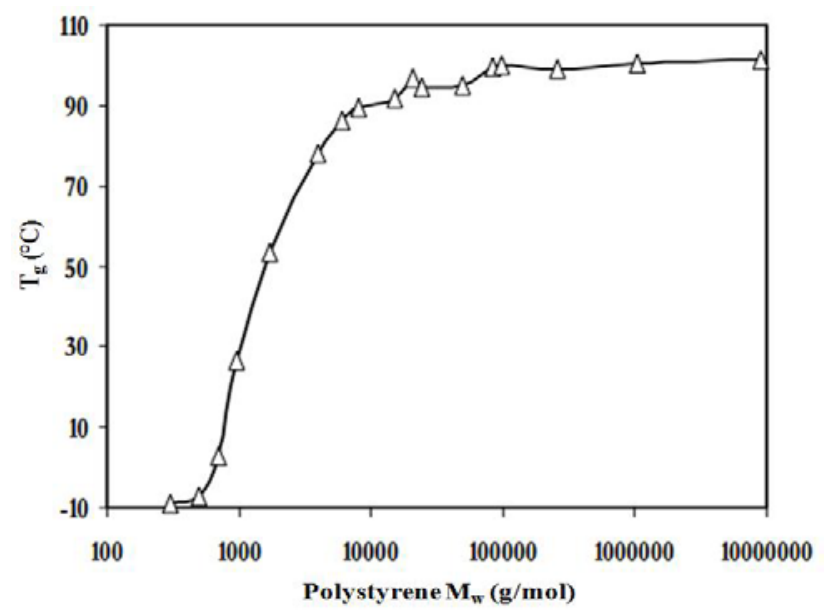

Figure 9. Bulk glass transition temperature as a function of polystyrene molecular weight. $T_{g}$ was determined using differential scanning calorimetry with a scan rate of $10^{\circ} \mathrm{C} / \mathrm{min}$. Taken from Ref. 44.

It is expected that wrinkle amplitude, and therefore skin thickness, of different molecular weight PS films should scale directly with the glass transition temperature. As figure 9 shows, there is a large change in $T_{g}$ as PS molecular weight goes from $1-10 \mathrm{~kg} / \mathrm{mol}$. For low PS molecular weight, $\mathrm{M}_{\mathrm{w}}=1 \mathrm{~kg} / \mathrm{mol}$, the $T_{\mathrm{g}}$ is at or just above room temperature and increases to about $90-100{ }^{\circ} \mathrm{C}$ for $\mathrm{M}_{\mathrm{w}} \geq 10 \mathrm{~kg} / \mathrm{mol}$. This means that during the spin casting, the low molecular weight PS films are very close to or slightly above their glass transition temperatures while high molecular weight films are $\sim 60{ }^{\circ} \mathrm{C}$ below their glass transition temperatures. Therefore, it makes sense that the low molecular weight PS films show very small wrinkle amplitudes because during the spinning process the PS stays in a rubbery state and no glassy skin layer is formed. Conversely, for high molecular weight PS films, a skin layer forms and its thickness grows with increasing $\mathrm{M}_{\mathrm{w}}$ due to the glass transition temperatures. Studies have shown that the $T_{g}$ of polymer can vary with film thickness, but this is only significant when the thickness is on the order of the molecular dimensions of the polymer. $^{48,49}$

The model above is sufficient to describe the behavior of the PS films up until a molecular weight threshold is crossed, which is demonstrated in figure 7 for both the wrinkles 
and pillars. The amplitude and wavelength should both scale directly with molecular weight, however when $\mathrm{M}_{\mathrm{w}}>105 \mathrm{~kg} / \mathrm{mol}$ there is a steady decrease in the wavelength and amplitude for both the pillars and wrinkles. The reason for this behavior stems from the fact that when $\mathrm{M}_{\mathrm{w}}>105 \mathrm{~kg} / \mathrm{mol}$, formation of the skin layer becomes dependent on a kinetic component. The high molecular weight chains are hindered by their weight and therefore cannot reorganize as fast as the low molecular weight films. This traps the high molecular weight films in a state where the skin layer hasn't had a chance to completely form since all films are spun for the same amount of time.

Above it was mentioned that the concentration of the casting solutions was changed in order to achieve a constant film thickness for all PS molecular weights. Initially, it might appear that this would affect the surface morphology, but as figure 10A shows, changing the casting solution concentration over the range of $1-4 \%$ has no effect on either the wavelength or amplitude of the wrinkle pattern formed.

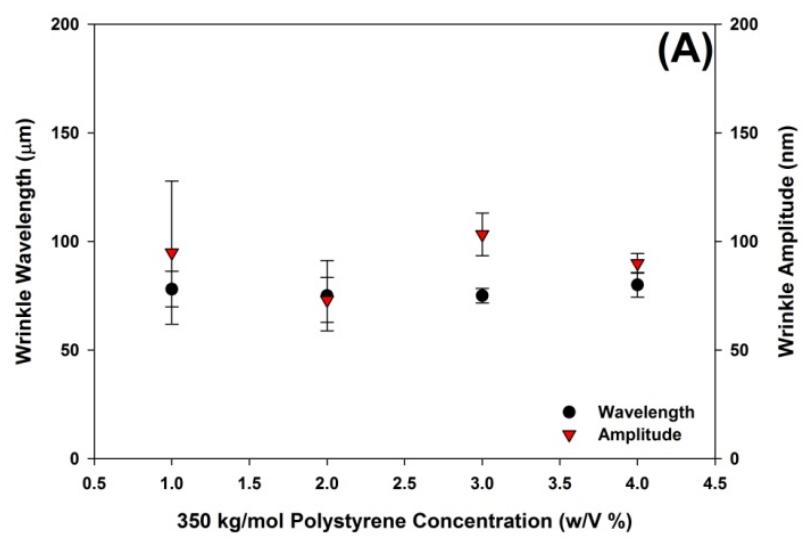




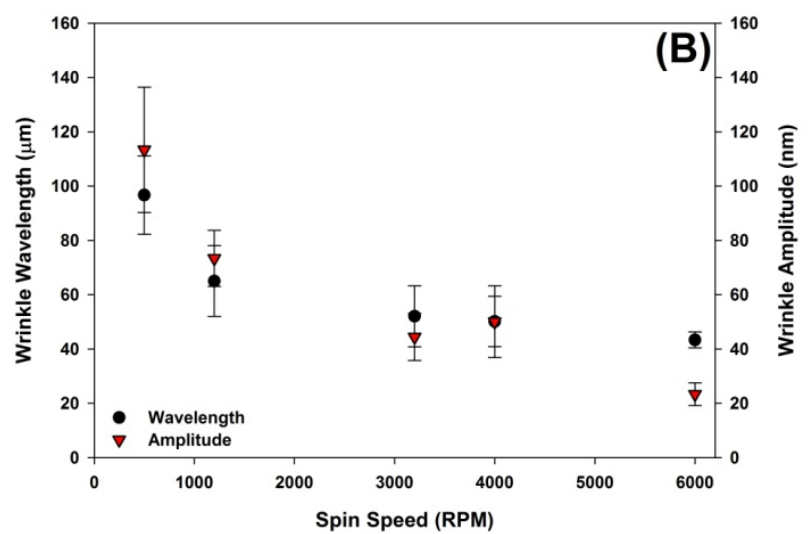

Figure 10. A) Average wrinkle wavelength and amplitude as a function of spin speed for a $2.0 \%$ PS solution. B) Average wrinkle wavelength and amplitude as a function of PS concentration, films spun at 1200 RPM. Polystyrene $M_{w}=350 \mathrm{~kg} / \mathrm{mol}$ for both A and B. Error bars represent a standard deviation of three measurements.

However, when the spin speed is altered there is a change in both the wrinkle wavelength and amplitude, as shown in figure 10B. This data supports the fact that there is a kinetic component involved with forming the wrinkles. When the spin speed is fast, the $350 \mathrm{~kg} / \mathrm{mol}$ PS film has even less time to reorganize and form a skin layer which leads to small wrinkles with a short wavelength. As the spin speed slows down, the heavy polymer chains have more time to move and a larger skin layer can form, leaving the surface with wrinkles of longer wavelengths with more amplitude.

\section{Conclusions}

The surface morphology of $300 \mathrm{~nm}$ spun cast polystyrene thin films as a function of PS molecular weight has been studied using optical profilometry. It was found the surface morphology changed a function of the molecular weight of polystyrene used to cast the film. For all PS films, the surface spontaneously forms two different structures depending on the location on the sample. On the extremities of the sample, radial wrinkle patterns are observed which transition into micron sized pillars at the center. The pillars are formed from interference of the wrinkle patterns. Due to the modulation in the glass transition temperature 
of polystyrene as a function of molecular weight, the characteristics of the wrinkle patterns, such as wrinkle wavelength and amplitude, can be controlled by choosing a particular molecular weight to cast the film with or by changing the spin speed used to cast the film. Control over these features is attributed to a rigid glassy PS layer forming over a still solvated, liquid-like PS layer underneath. When the liquid-like layer dries completely, the rigid layer buckles giving way to a wrinkle surface. However, when the PS molecular weight passes a critical limit, kinetic effects in the forming of the rigid PS layer must be considered. Changing the spin speed in heavy PS films also has been shown to change the wrinkle characteristics and adds another layer of control over the surface morphology.

\section{ASSOCIATED CONTENT}

\section{Supporting Information.}

The Supporting Information is available free of charge on the ACS Publications website. Line scans of wrinkle profile with sinusoidal fits for wavelength and amplitude values.

\section{Notes}

The authors declare no competing financial interest.

\section{ACKNOWLEDGEMENT}

This material is based upon work supported by the U.S. Department of Homeland Security, Science and Technology Directorate, Office of University Programs, under 
Grant Award 2013-ST-061-ED0001. The views and conclusions contained in this document are those of the authors and should not be interpreted as necessarily representing the official policies, either expressed or implied, of the U.S. Department of Homeland Security.

\section{REFERENCES}

(1) Su, J.; Chen, J., Synthetic porous materials applied in hydrogenation reactions. Microporous Mesoporous Mater., 2017, 237, 246-259.

(2) Pan, L.; Qiu, H.; Dou, C.; Li, Y.; Pu, L.; Xu, J.; Shi, Y., Conducting Polymer Nanostructures: Template Synthesis and Applications in Energy Storage. Int. J. Mol. Sci., 2010, 11, 2636-2657.

(3) Share, K.; Westover, A.; Li, M.; Pint, C. L., Surface Engineering of Nanomaterials for Improved Energy Storage - A Review. Chem. Eng. Sci., 2016, 154, 3-19.

(4) Gor, G. Y., Adsorption Stress Changes the Elasticity of Liquid Argon Confined in a Nanopore. Langmuir, 2014, 30, 13564 - 13569.

(5) Ghosh, J.; Faller, R., Comparing the Density of States of Binary Lennard-Jones Glasses in Bulk and Film. J. Chem. Phys., 2008, 128, 124509.

(6) Zhan, D.; Han, L.; Zhang, J.; Shi, H.; Zhou, J. Z.; Tian, Z. W.; Tian, Z. Q., Confined Chemical Etching for Electrochemical Machining with Nanoscale Accuracy. Acc. Chem. Res., 2016, 49, 2596 - 2604. 
(7) Huang, Z; Geyer, N.; Werner, P.; Boor, J. de; Gösele, U., Metal Assisted Chemical Etching of Silicon: A Review. Adv. Mater., 2011, 23, 285 - 308.

(8) Chen, Y.; Pépin, A., Nanofabrication: Conventional and Nonconventional Methods. Electrophoresis, 2001, 22, 187 - 207.

(9) Fourche, G., An Overview of the Basic Aspects of Polymer Adhesion. Part I: Fundamentals, Polym. Eng. \& Sci., 1995, 35, 957 - 967.

(10) Adamson, A. W.; Gast, A. P., Physical Chemistry of Surfaces, $6^{\text {th }}$ edition, John Wiley \& Sons, New York, 1997.

(11) Genet, C.; Ebbesen, T. W., Light in Tiny Holes. Nature, 2007, 445, 39 - 46.

(12) Levitsky, I. A.; Euler, W. B.; Tokranova, N.; Rose, A., Fluorescent Polymer Porous Silicon Microcavity Devices for Explosives Detection. Appl. Phys. Lett., 2007, 90, 041904.

(13) Lalanne, P.; Sauvan, C.; Hugonin, J. P., Photon Confinement in Photonic Crystal Nanocavities. Laser \& Photonics Reviews, 2008, 2, 514 - 526.

(14) Hsiao, Y. S.; Charan, S.; Wu, F. Y.; Chien, F. C.; Chu, C. W.; Chen, P.; Chen, F. C., Improving the Light Trapping Efficiency of Plasmonic Polymer Solar Cells through Photon Management. J. Phys. Chem. C, 2012, 116, 20731 - 20737.

(15) Matoian, M. A.; Sweetman, R.; Hall, E. C.; Albanese, S.; Euler, W. B., Light Trapping to Amplify Metal Enhanced Fluorescence with Application for Sensing TNT. J. Fluoresc., 2013, 23, 877- 880.

(16) Zhou, W.; Liu, R.; Tang, D.; Wang, X.; Fan, H.; Pan, A.; Zhang, Q.; Wan, Q.; Zou, B., Luminescence and Local Photonic Confinement of Single ZnSe:Mn 
Nanostructure and the Shape Dependent Lasing Behavior. Nanotechnology, 2013, 24, 055201.

(17) Mischok, A.; Brückner, R.; Sudzius, M.; Reinhardt, C.; Lyssenko, V. G.; Fröb, H.; Leo, K., Photonic Confinement in Laterally Structured Metal-Organic Microcavities. Appl. Phys. Lett., 2014, 105, 051108.

(18) Khang, D. Y.; Jiang, H.; Huang, Y.; Rogers, J. A., A Stretchable Form of SingleCrystal Silicon for High-Performance Electronics on Rubber Substrates. Science, 2006, 311, 208-212.

(19) Kim, D. H.; Ahn, J. H.; Choi, W. M.; Kim, H. S.; Kim, T. H.; Song, J.; Huang, Y. Y.; Liu, Z.; Lu, C.; Rogers, J. A., Stretchable and Foldable Silicon Integrated Circuits. Science, 2008, 320, 507-511.

(20) Reiter, G., Unstable Thin Polymer Films - Rupture and Dewetting Processes. Langmuir, 1993, 9, 1344 - 1351.

(21) Sharma, A.; Reiter, G., Instability of Thin Polymer Films on Coated Substrates: Rupture, Dewetting, and Drop Formation. J. Colloid Interface Sci., 1996, 178, 383 399.

(22) Müller-Buschbaum, P.; Bauer, E.; Wunnicke, O.; Stamm, M., The Control of Thin Film Morphology by the Interplay of Dewetting, Phase Separation, and Microphase Separation. J. Phys.: Condens. Matter, 2005, 17, S363 - S386.

(23) Wu, N.; Pease III, L. F.; Russel, W. B., Toward Large-Scale Alignment of Electrohydrodynamic Patterning of Thin Polymer Films. Adv. Funct. Mater., 2006, 16, $1992-1999$. 
(24) Schaffer, E.; Thurn-Albrecht, T.; Russel, T. P.; Steiner, U., Electrically Induced Structure Formation and Pattern Transfer. Nature, 2000, 403, 874 - 877.

(25) Bunz, U. H. F., Breath Figures as a Dynamic Templating Method for Polymers and Nanomaterials. Adv. Mater., 2006, 18, 973 - 989.

(26) Hernandez-Guerrero, M.; Stenzel, M. H., Honeycomb Structure Polymer Films via Breath Figures. Polym. Chem., 2012, 3, 563 - 577.

(27) Schaffer, E.; Harkema, S.; Blossey, R.; Steiner, U., Temperature Gradient Induced Instability in Polymer Films. Europhys. Lett., 2002, 60, 255 - 261.

(28) Schaffer, E.; Harkema, S.; Roerdink, M.; Blossey, R.; Steiner, U., Thermomechanical Lithography: Pattern Replication using a Temperature Gradient Driven Instability. Adv. Mater., 2003, 15, 514 - 517.

(29) Xue, L.; Zhang, J.; Han, Y., Phase Separation Induced Ordered Patterns in Thin Polymer Blend Films. Prog. Polym. Sci., 2012, 37, 564 - 594.

(30) Reiter, G.; Castelein, G.; Hoerner, P.; Riess, G.; Blumen, A.; Sommer, J. U., Nanometer-Scale Surface Patterns with Long Range Order Created by Crystallization of Diblock Copolymers. Phys. Rev. Lett., 1999, 83, 3844 - 3847.

(31) Fournier, A. C.; Cumming, H.; McGrath, K. M., Assembly of Two- and Threedimensionally Patterned Silicate Materials Using Responsive Soft Templates. Dalton Trans., 2010, 39, $6524-6531$.

(32) Shen, M., Nano-structuring Solid Surfaces with Femtosecond Laser Irradiations for Applications. Mod. Phys. Lett. B, 2010, 24, 257 - 269. 
(33) Childs, W. R.; Nuzzo, R. G., Decal Transfer Microlithography: A New SoftLithographic Patterning Method. J. Am. Chem. Soc., 2002, 124, 13583 - 13596.

(34) Meng, J.; Xie, J.; Han, X.; Lu, C., Surface Wrinkling on Polydopamine Film. App. Surf. Sci., 2016, 371, 96 - 101.

(35) Feng, C.; Yi, Z.; Dumee, L. F.; Garvey, C. J.; She, F.; Lin, B.; Lucas, S.; Schultz, J.; Gao, W.; Peng, Z.; et.al., Shrinkage Induced Stretchable Micro-Wrinkled Reduced Graphene Oxide Composite with Recoverable Conductivity. Carbon, 2015, 93, 878 886.

(36) Chapman, C. T.; Paci, J. T.; Lee, W. K.; Engel, C. J.; Odom, T. W.; Schatz, G. C., Interfacial Effects on Nanoscale Wrinkling in Gold-Covered Polystyrene. ACS Appl. Mater. Interfaces, 2016, 8, 24339 - 24344.

(37) Torres, J. M.; Stafford, C. M.; Vogt, B. D., Impact of Molecular Mass on the Elastic Modulus of Thin Polystyrene Films. Polymer, 2010, 51, 4211 - 4217.

(38) Bornside, D. E.; Macosko, C. W.; Scriven, L. E., Spin Coating of a PMMA/Chlorobenzene Solution. J. Electrochem. Soc., 1991,138, 317 - 320.

(39) Kim, S. T.; Kim, T. G.; Cho, H.; Yoon, S. J.; Kim, H. S.; Kim, J. K., Thickness Dependence of Properties of ITO Films Deposited on PET Substrates. J. Nanosci. Nanotechnol., 2016, 16, $1852-1854$.

(40) Raja, S.; Bellan, C. S.; Sundaram, S.; Subramani, G.; Rajamani, R., Thickness Dependence on Structural, Dielectric, and AC Conduction Studied of Vacuum Evaporated Sr Doped $\mathrm{BaTiO}_{3}$ Thin Films. Optik, 2016, 127, 3200 - 3205. 
(41) He, L.; Zhang, L.; Liang, H., Microdomain Morphology of Lamella-Forming Diblock Copolymer Confined in a Thin Film. J. Polym. Sci., Part B: Polym. Phys., 2008, 47, $1-10$.

(42) Xia, F.; Razavi, B.; Xu, H.; Cheng, Z. Y.; Zhang, Q. M., Dependence of Threshold Thickness of Crystallization and Film Morphology on Film Processing Conditions in Poly(vinylidene fluoride-trifluroethylene) Copolymer Thin Films. J. Appl. Phys., 2002, 92, $3111-3115$.

(43) Rodgríguez-Hernández, J., Wrinkled Interfaces: Taking Advantage of Surface Instabilities to Pattern Polymer Surfaces. Prog. Polym. Sci., 2015, 42, 1 - 41.

(44) Li, B.; Cao, Y. P.; Feng, X. Q.; Gao, H., Mechanics of Morphological Instabilities and Surface Wrinkling in Soft Materials: A Review. Soft Matter, 2012, 8, $5728-5745$.

(45) Ramanathan, M.; Lokitz, B. S.; Messman, J. M.; Stafford, C. M.; M.Kilbey II, S., Spontaneous Wrinkling in Azlactone-Based Functional Polymer Thin Films in 2D and 3D Geometries for Guided Nanopatterning. J. Mater. Chem. C, 2013, 1, 2097-2101.

(46) Pauchard, L.; Allain, C., Buckling Instability Induced by Polymer Solution Drying. Europhys. Lett., 2003, 62, 897 - 903.

(47) Polymer Sciences Inc. Glass Transition Temperature of Polystyrene. https://www.polymersource.com/dataSheet/P8095-S.pdf (accessed Nov, 2016). 
(48) Keddie, J. L.; Jones, R. A. L.; Cory, R. A., Interface and Surface Effects on the Glass-Transition Temperature in Thin Polymer Films. Faraday Discuss., 1994, 98, 219 230.

(49) Alcoutlabi, M. and McKenna, G. B., Effects of Confinement on Material Behavior at the Nanometer Sized Scale. Condens. Matter, 2005, 17, R461 - R524. 


\title{
Surface Morphology Dependence on Molecular Weight in Polystyrene Thin
}

Films: Formation of Periodic Structures by Spin-Casting

\author{
Matthew Mullen and William B. Euler* \\ Department of Chemistry
}

140 Flagg Road

Kingston, RI 02881

\section{Supporting Information}

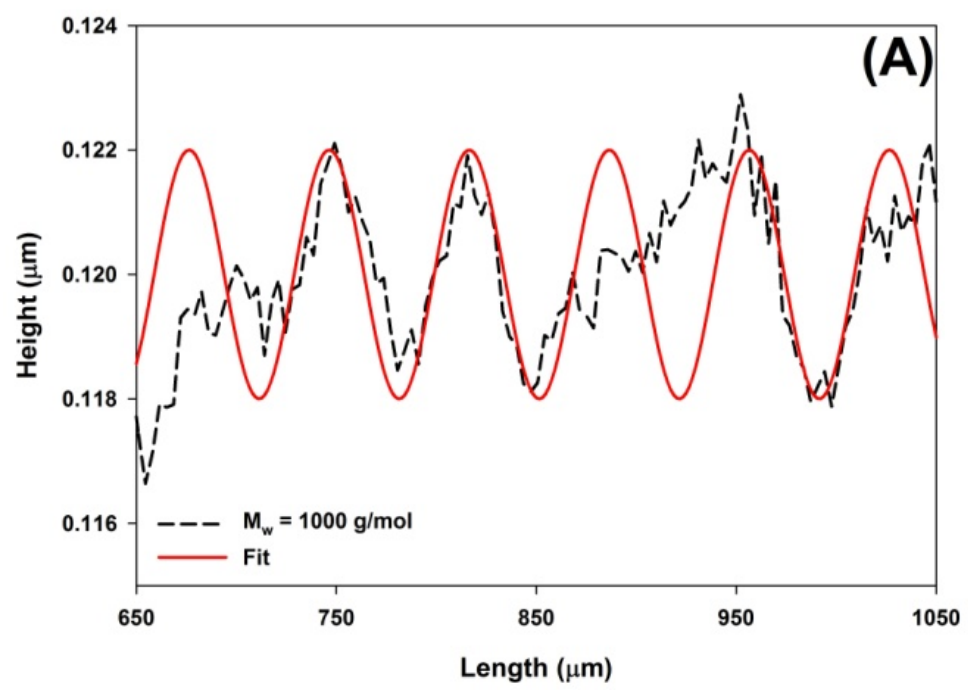



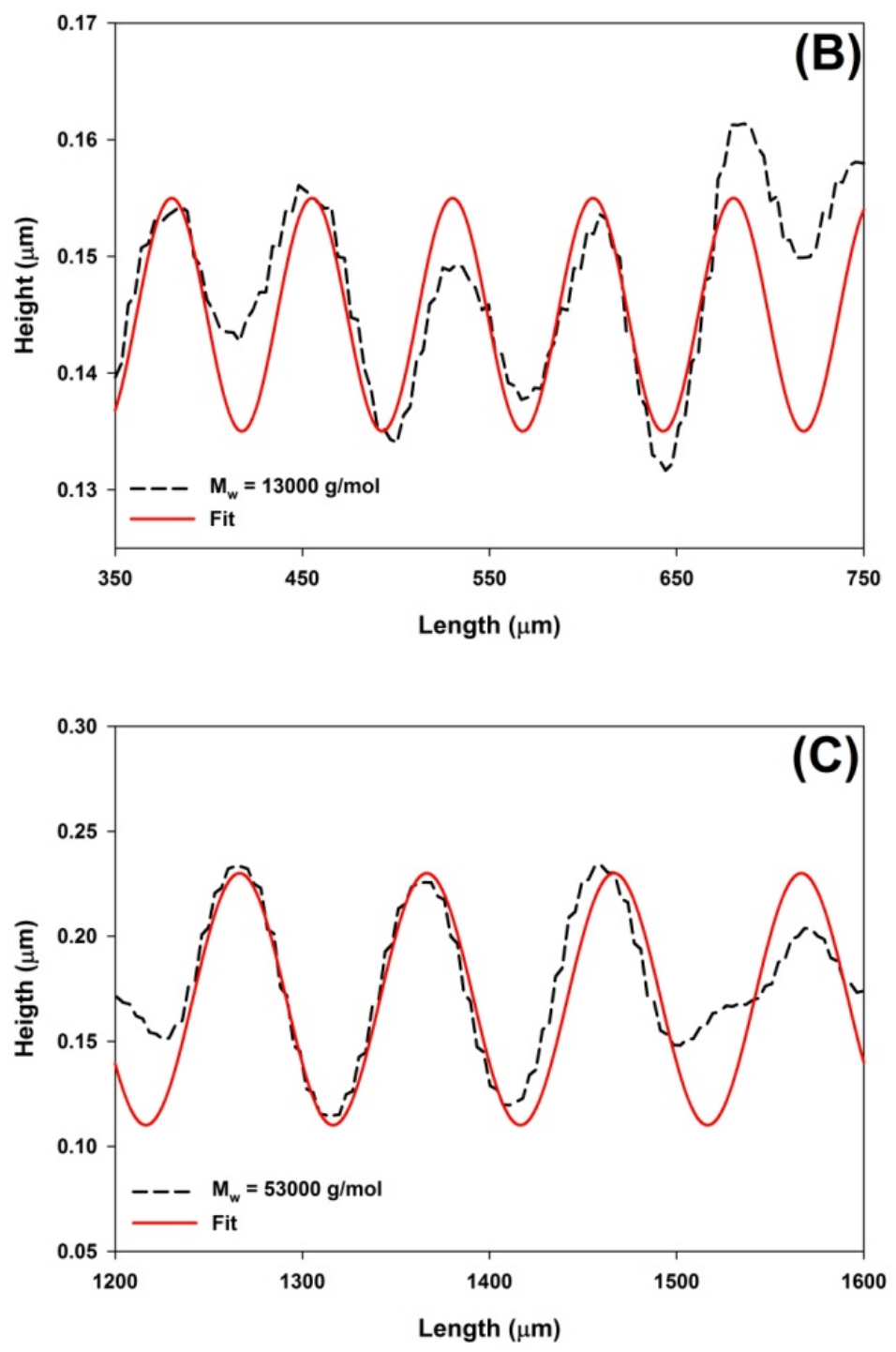

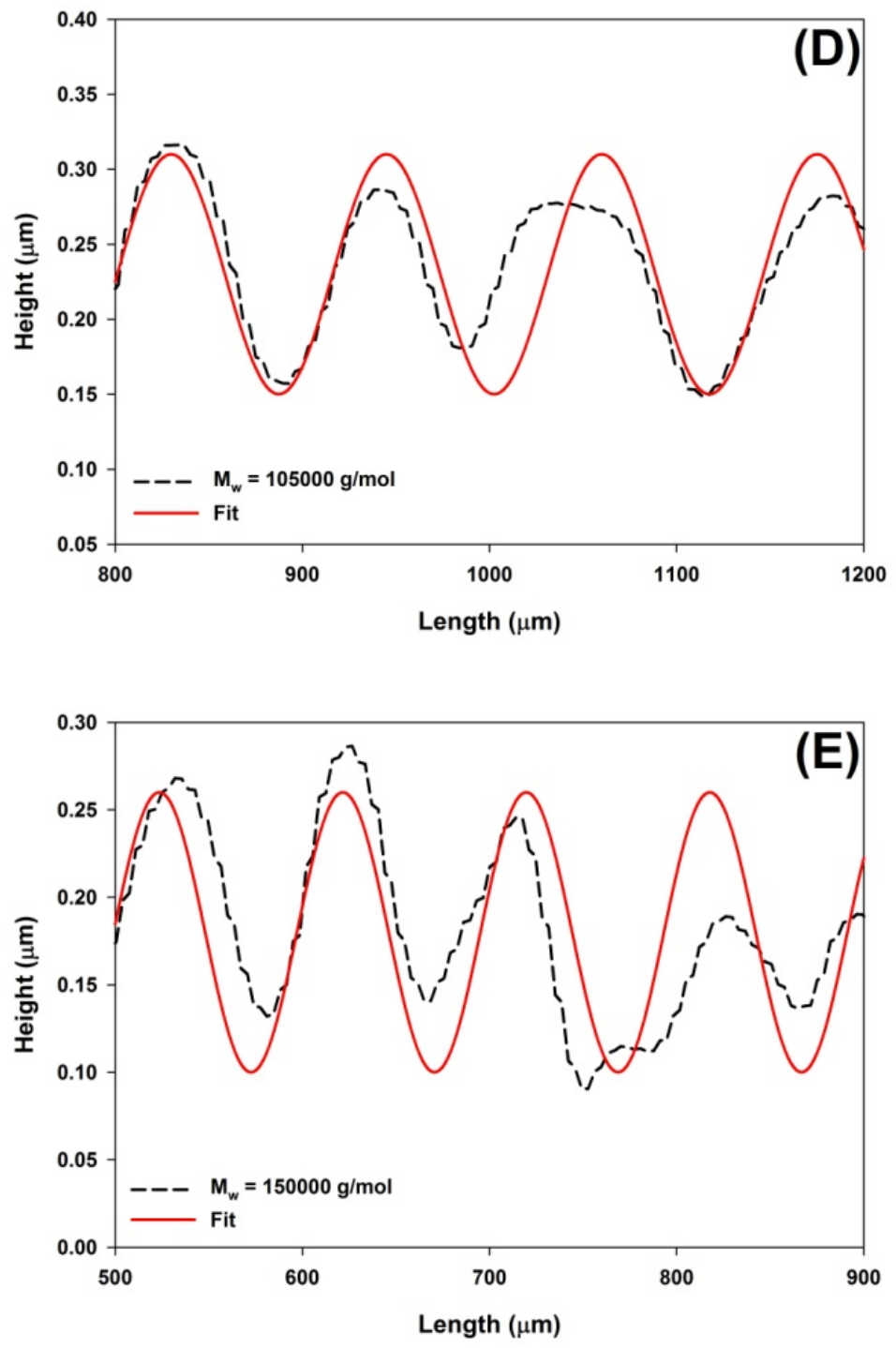

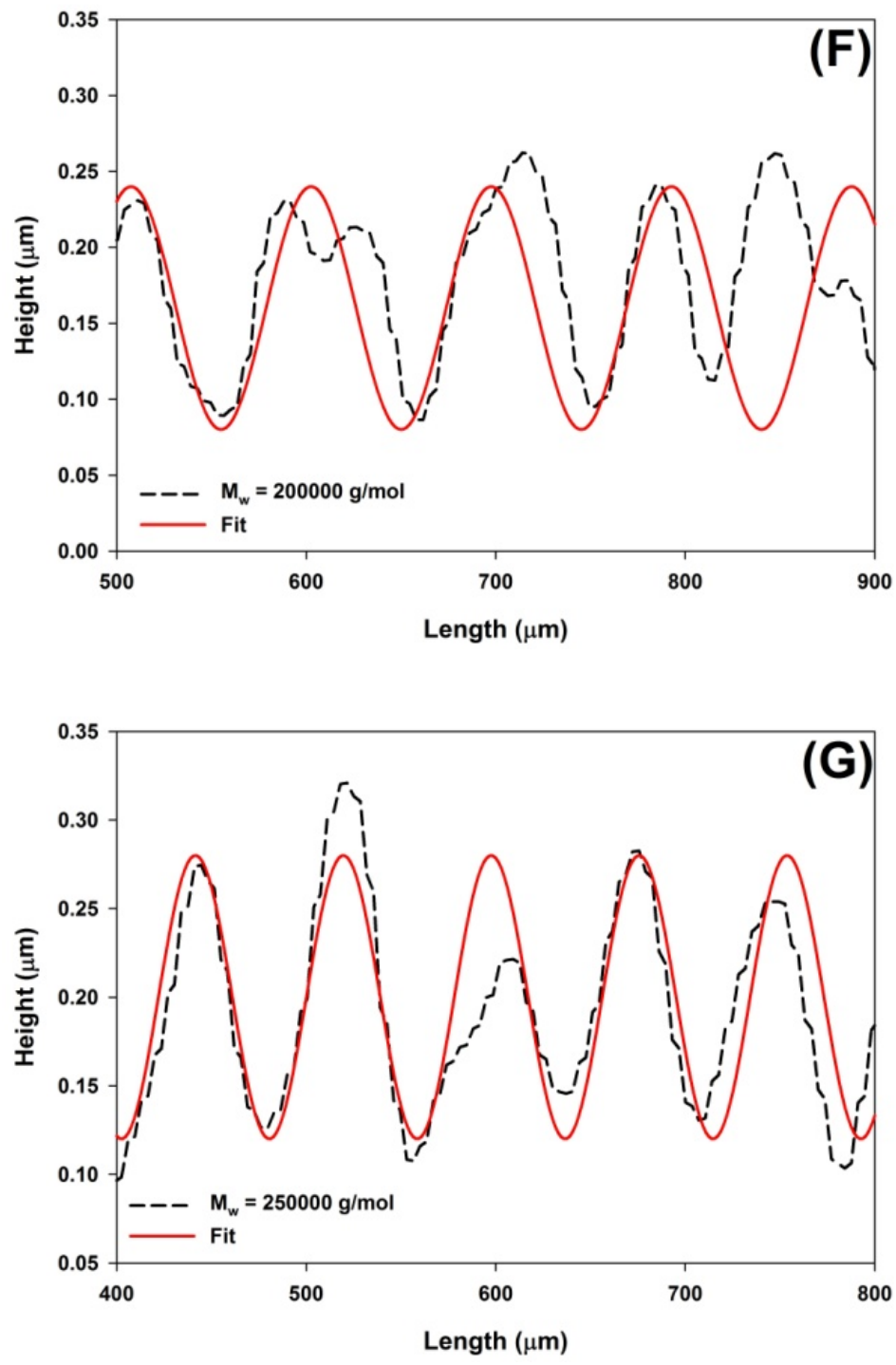

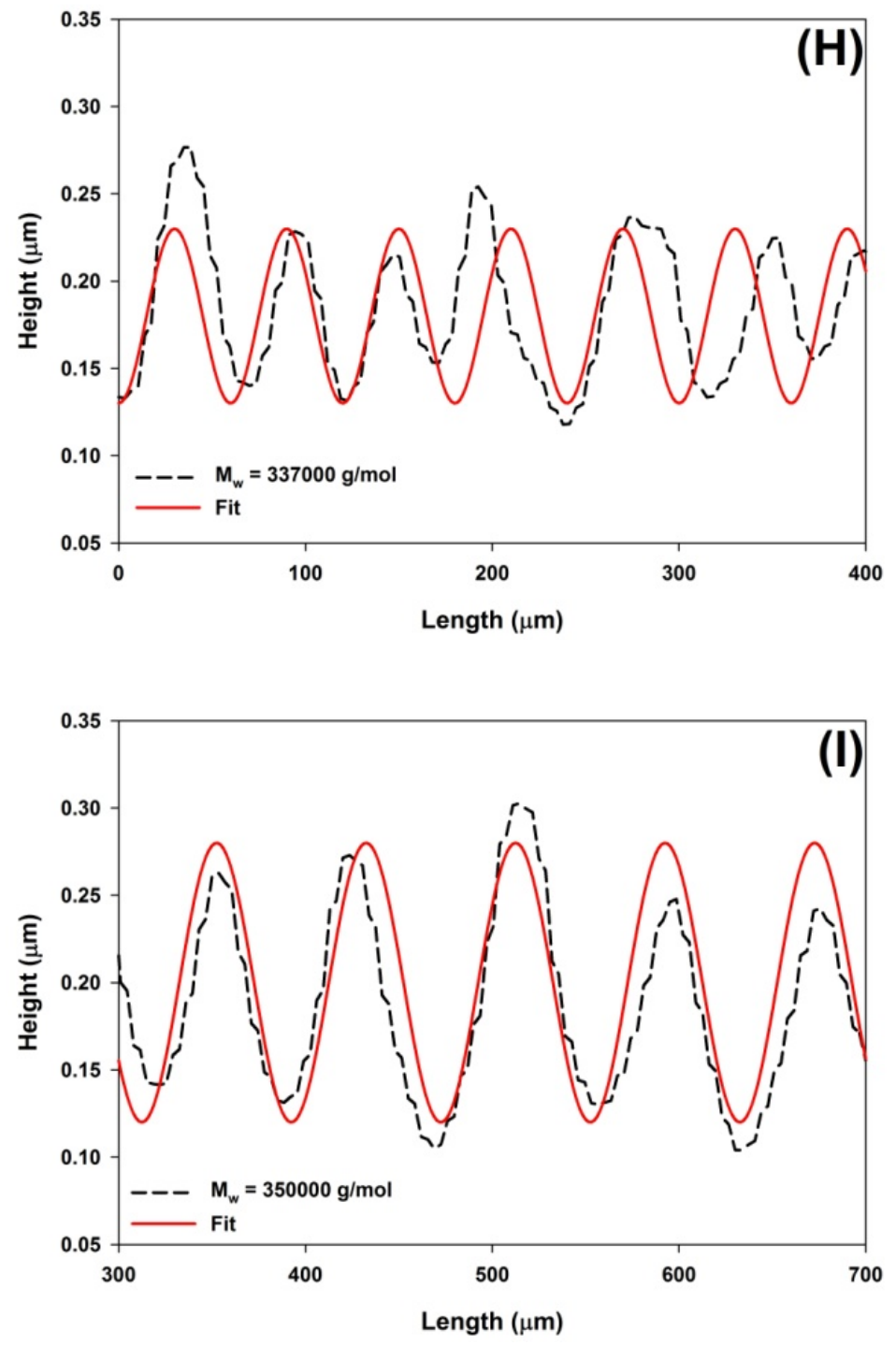

Figure S1A-S1I. Line scan profiles of the wrinkled polystyrene surface. A) $\mathrm{M}_{\mathrm{w}}=$ $\left.\left.1000 \mathrm{~g} / \mathrm{mol}, \mathrm{B}) \mathrm{M}_{\mathrm{w}}=13000 \mathrm{~g} / \mathrm{mol}, \mathrm{C}\right) \mathrm{M}_{\mathrm{w}}=53000 \mathrm{~g} / \mathrm{mol}, \mathrm{D}\right) \mathrm{M}_{\mathrm{w}}=105000 \mathrm{~g} / \mathrm{mol}, \mathrm{E}$ ) $\left.\left.\left.\mathrm{M}_{\mathrm{w}}=150000 \mathrm{~g} / \mathrm{mol}, \mathrm{F}\right) \mathrm{M}_{\mathrm{w}}=200000 \mathrm{~g} / \mathrm{mol}, \mathrm{G}\right) \mathrm{M}_{\mathrm{w}}=250000 \mathrm{~g} / \mathrm{mol}, \mathrm{H}\right) \mathrm{M}_{\mathrm{w}}=337000$ $\mathrm{g} / \mathrm{mol}$, and I) $\mathrm{M}_{\mathrm{w}}=350000 \mathrm{~g} / \mathrm{mol}$. 

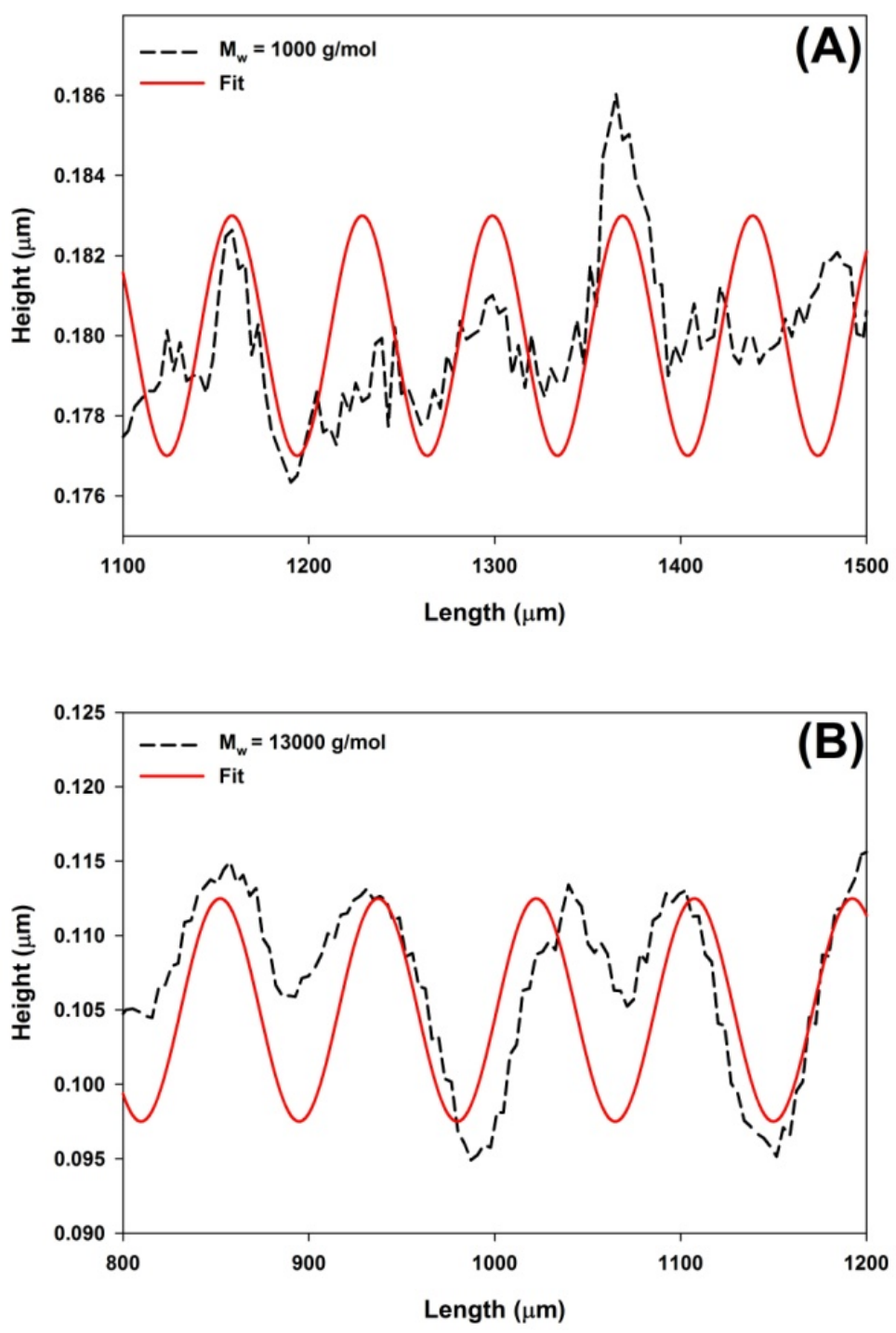

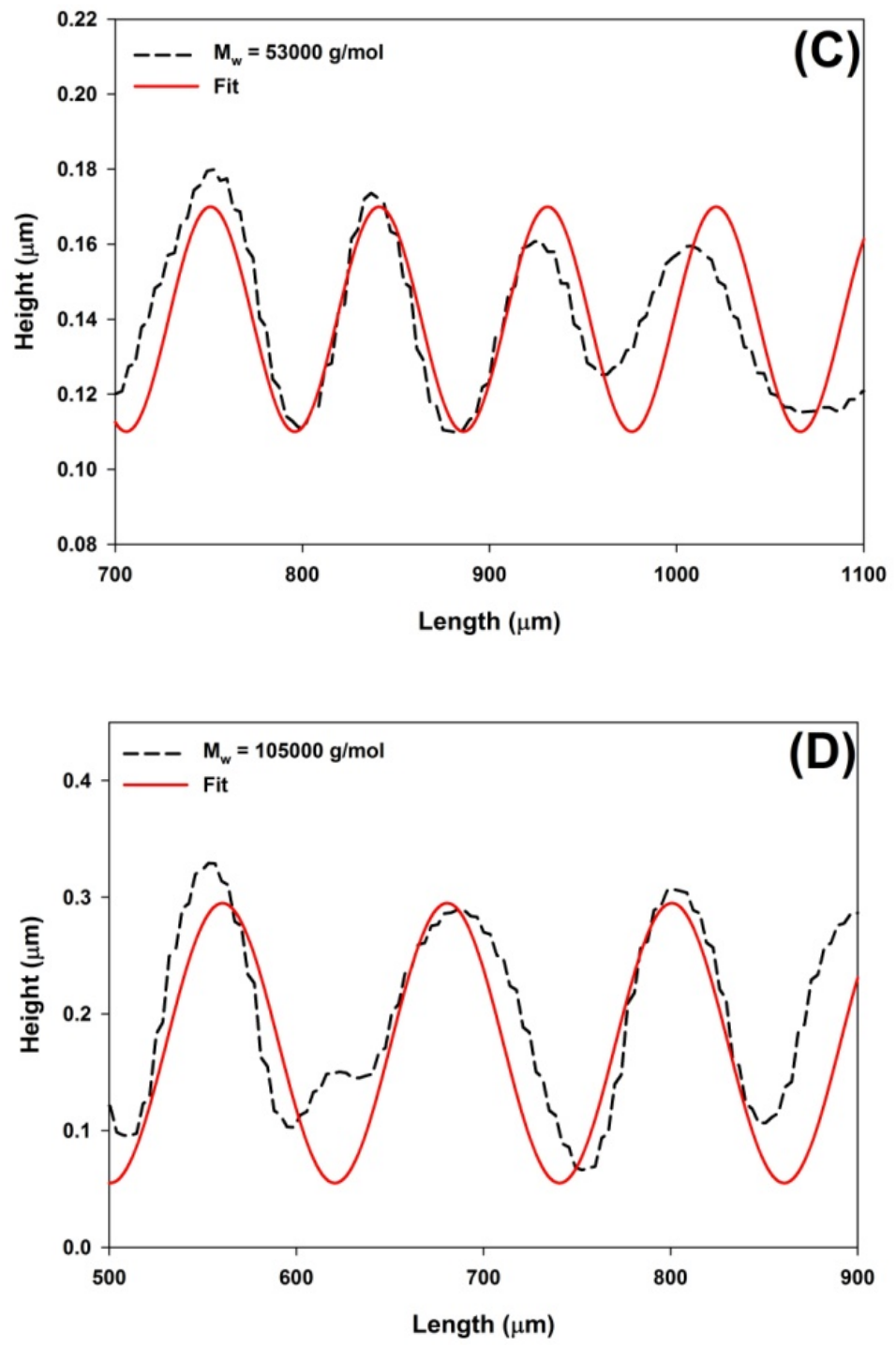

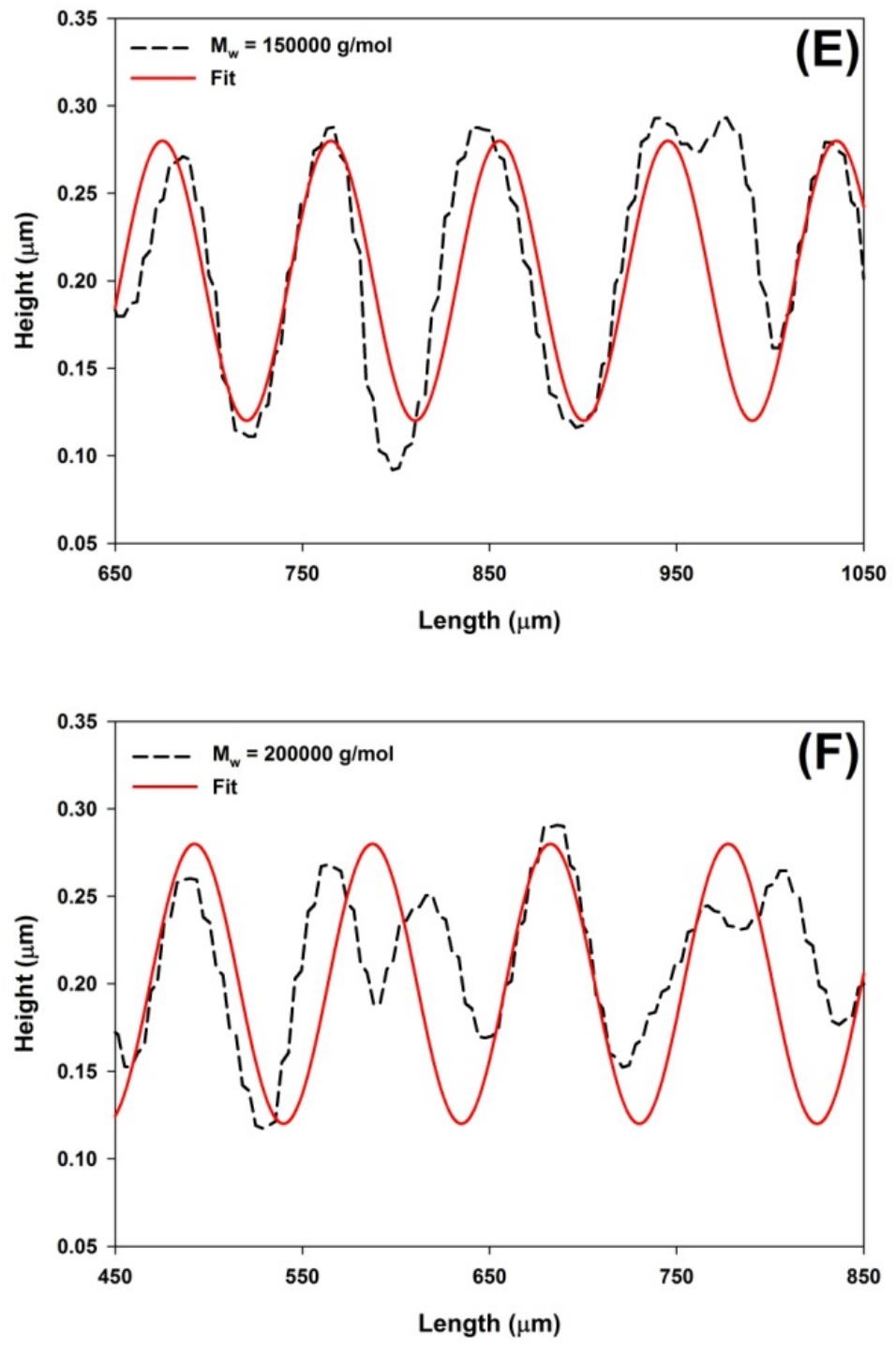

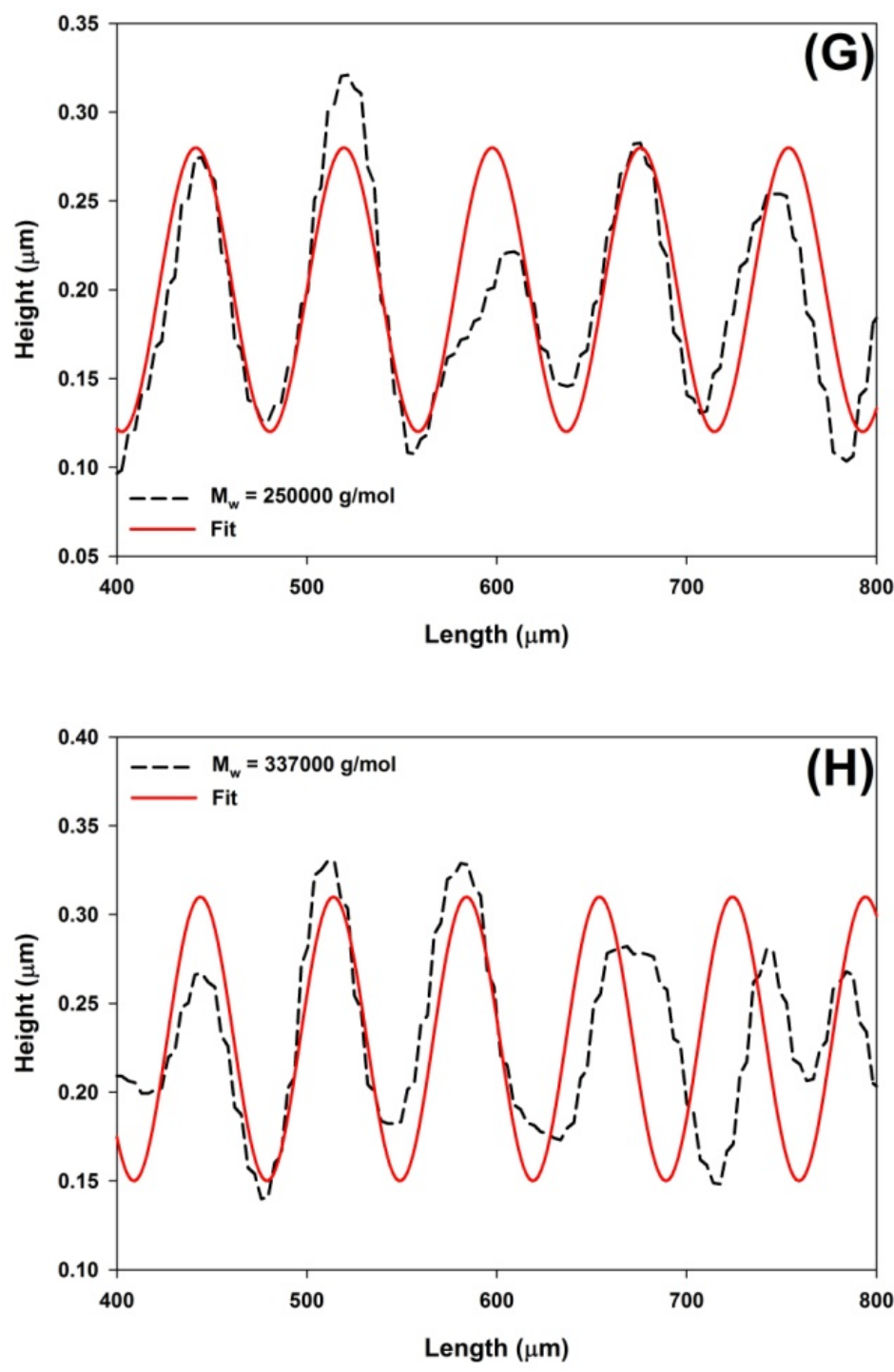


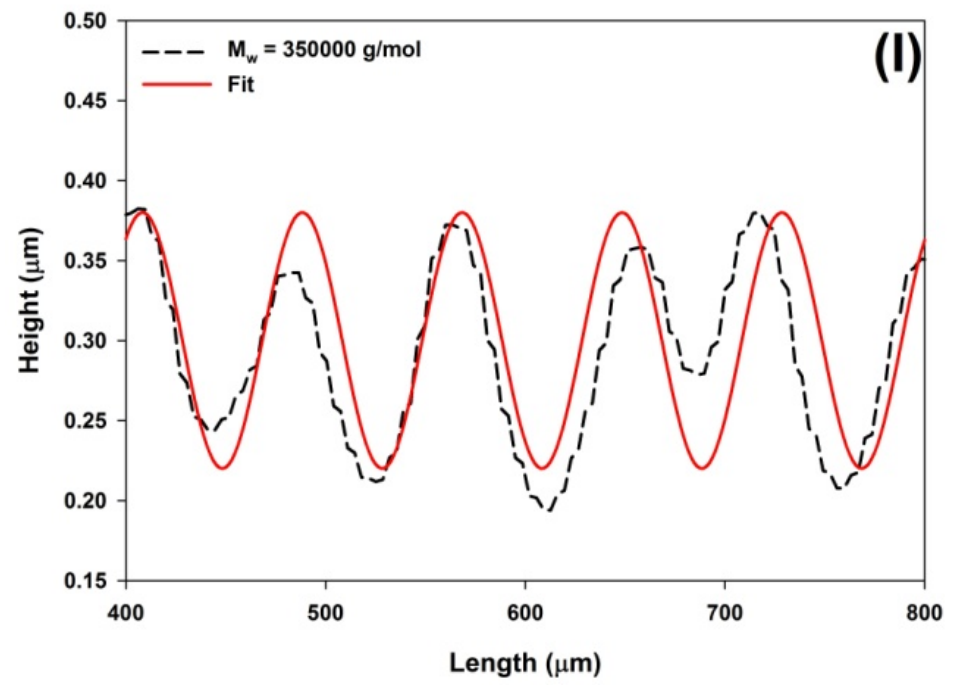

Figure S2A-S2I. Line scan profiles of the pillared polystyrene surface. A) $\mathbf{M}_{w}=1000$ $\mathrm{g} / \mathrm{mol}$, B) $M_{\mathrm{w}}=13000 \mathrm{~g} / \mathrm{mol}$, C) $M_{\mathrm{w}}=53000 \mathrm{~g} / \mathrm{mol}$, D) $\left.M_{\mathrm{w}}=105000 \mathrm{~g} / \mathrm{mol}, \mathrm{E}\right) \mathrm{M}_{\mathrm{w}}=$ $150000 \mathrm{~g} / \mathrm{mol}$, F) $\left.\left.\mathrm{M}_{\mathrm{w}}=200000 \mathrm{~g} / \mathrm{mol}, \mathrm{G}\right) \mathrm{M}_{\mathrm{w}}=250000 \mathrm{~g} / \mathrm{mol}, \mathrm{H}\right) \mathrm{M}_{\mathrm{w}}=337000$ $\mathrm{g} / \mathrm{mol}$, and I) $\mathrm{M}_{\mathrm{w}}=350000 \mathrm{~g} / \mathrm{mol}$. 


\title{
CHAPTER 3
}

A Spectroscopic Study of Charged Xanthene Dyes on a Polystyrene Surface: An Investigation into Potential Ion- $\pi$ Interactions

\author{
Matthew Mullen, Nolan Fontaine, and William B. Euler* \\ Department of Chemistry \\ 140 Flagg Road \\ Kingston, RI 02881
}

The following is in preparation for submission to the Journal of Physical Chemistry C and is presented here in manuscript format. 


\begin{abstract}
Thin films of three differently charged xanthene dyes: rhodamine 6G (Rh6G, cationic), fluorescein 27 (F27, neutral), and disodium fluorescein (DSF, anionic) were cast onto a polystyrene (PS) coated glass substrate to investigate the possibility of ion$\pi$ interactions. Absorbance spectroscopy was used to determine the state of the dyes on the PS surface. Deconvolution of the spectra of films formed from the lowest casting concentration revealed three peaks for all dyes: a high energy shoulder on the monomer peak and a low energy peak. The shift of the low energy peak from the monomer peak was used as an indication of interaction strength of that species and followed the trend of Rh6G > DSF > F27. Increase in the interaction energy is attributed to stabilization from ion- $\pi$ interactions. Steady-state emission spectra and excited state lifetime measurements were performed on all films. Lifetime measurements could only be made for the charged dyes and revealed the formation of a weakly emissive exciplex for both Rh6G and DSF which quenches the overall emission from those films. No evidence of the exciplex is found for the F27 films and as the F27 film thickness increase so does the emission intensity.
\end{abstract}

\title{
Introduction
}

Since 1981, when Kebarle and co-workers ${ }^{1}$ showed that $\mathrm{K}^{+}$ions in the gas phase bind stronger to benzene than to water, cation- $\pi$ interactions have been studied extensively both from a theoretical standpoint and experimentally. ${ }^{2-4}$ Investigations into the cation- $\pi$ interaction have shown that it is not restricted only to atomic ions, but molecular ions like ammonium and larger molecules as well. Dougherty et.al. ${ }^{5}$ went 
on to show using quaternary amines that cation- $\pi$ interactions are still energetically significant in the solution phase and are responsible for a number of biological interactions in proteins. In more recent studies, the potential for cation- $\pi$ interactions in the solid phase has been investigated, however most of these studies have been on protein crystal structures. ${ }^{6,7}$ In the field of adhesion, the mechanism of mussels adhering to underwater substrates is being studied and it has been proposed that cation- $\pi$ interactions play a large role in that interaction. ${ }^{8,9}$ The discovery of cation- $\pi$ interactions led researchers to look for evidence of the opposite case of anion- $\pi$ interactions and a comprehensive review of anion- $\pi$ interactions has been given by Giese et.al.. ${ }^{10}$

It is textbook material that the characteristic emission of fluorophores is strongly dependent on its interactions with its surrounding media. ${ }^{11}$ In solution, fluorescence emission wavelength can shift hundreds of nanometers depending on the interactions a fluorophore has with solvent molecules. The dielectric environment of the solvent typically dictates which direction the emission will shift, and the extent of the shift is dependent on how strong electrostatic and noncovalent interactions between the solvent molecule and fluorophore are. In the past, researchers have only considered noncovalent interactions such as hydrogen bonding, van der Waals forces, or hydrophilic/hydrophobic interactions, but now the ion- $\pi$ interactions must be considered as well. There are reports of cation- $\pi$ interactions being used in turn on/off fluorescence for the detection of metal ions, ${ }^{12-16}$ however these are mostly done in the solution phase, with the metal ion interacting with the aromatic rings of the dye resulting in quenched emission. 


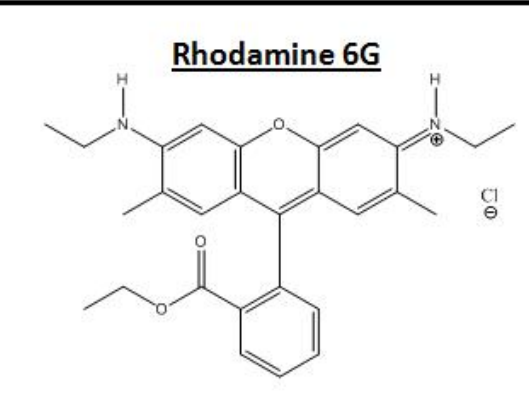

Disodium Fluorescein

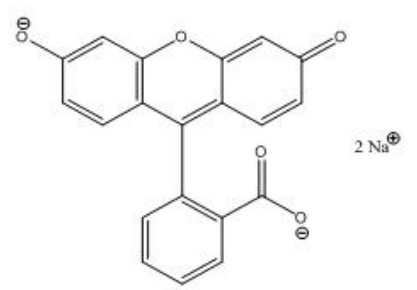

Fluorescein 27

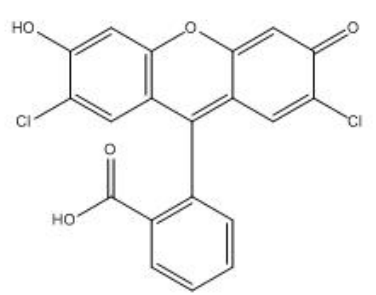

Polystyrene

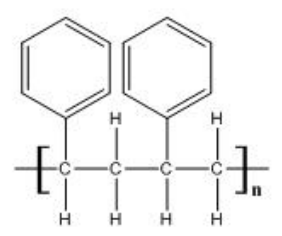

Figure 1. Molecular structure of fluorophores and polymer.

Polystyrene (PS) is a common polymer used in testing cation- $\pi$ interactions due to its abundant source of aromatic rings. ${ }^{8,17}$ Typically, a thin film of PS is fabricated then used as a surface for probing cation- $\pi$ interactions. Additionally, Bertrand et.al. ${ }^{18}$ showed using time of flight secondary ion mass spec. that in spin coated thin films of PS the phenol group constitutes a large majority of the surface. Herein we report on a layered thin film system that consist of a PS coated substrate which then has a thin film of an organic dye coated on top. The three dyes used in this study: rhodamine 6G (Rh6G), fluorescein 27 (F27), and disodium fluorescein (DSF), are chosen due to their similar chemical structure with a central xanthene plane. Of the three, Rh6G and DSF exist as molecular ions in solution. Rh6G is a monovalent cation, DSF is a divalent anion, and F27 serves as a neutral dye. By studying the 
changes in the UV/Vis and fluorescence spectra of these dyes as a function of their film thickness, the potential for solid phase ion- $\pi$ interactions can be probed.

\section{Experimental Methods}

Microscope glass slides were cut into dimensions of $3.75 \mathrm{~cm} \times 1.75 \mathrm{~cm}$. The samples were then placed into a beaker of 95\% ethanol (EtOH, Pharmaco-Aaper). The solution was sonicated for 15 mins. The cut glass slides were rinsed with water and sonicated for an additional 15 minutes, then dried with $\mathrm{N}_{2}$ gas. Polystyrene (PS, Sigma-Aldritch) $\mathrm{M}_{\mathrm{w}}=350 \mathrm{~kg} / \mathrm{mol}(\mathrm{PDI}=2.05)$ was dissolved in tetrahydrafuran (THF, Sigma Aldrich, HPLC Grade) and sonicated for 1 hour. Once all the PS is in solution, the PS films are made by spin casting. The glass substrate was placed on the stage and a $500 \mu \mathrm{L}$ aliquot of PS solution was deposited in the center. The substrate was spun for $45 \mathrm{~s}$ at room temperature then placed in an oven at $60{ }^{\circ} \mathrm{C}$ for $1 \mathrm{~min}$ to evaporate remaining solvent.

The film thickness of the PS was determined using a Filmetrics F40 microscope. A reflectance spectrum was recorded then fit with a model of given thickness and refractive index. The reflection spectrum was recorded with a tungstenhalogen light source over the range of 400-900 $\mathrm{nm}$.

After characterizing the PS films, the samples were then coated with a thin film of fluorescent dye. The three dyes used were Rh6G (Acros, 99\%), F27 (Lambda Physik, 99\%), and DSF (Exciton, 99\%) all of which were dissolved in EtOH. The

concentration range used for the dyes ranged from $1.0 \times 10^{-5} \mathrm{M}$ to $1.0 \times 10^{-3} \mathrm{M}$. The fluorescent dye films were made by spin casting a $250 \mu \mathrm{L}$ aliquot onto the PS coated 
glass slides. The samples were spun at 1800 RPM for $45 \mathrm{~s}$, and then they were left to air dry.

The absorbance spectrum of each sample was collected on a Perkin-Elmer Lambda 1050 UV/Vis/NIR spectrometer with $2.0 \mathrm{~nm}$ slit widths. Before the fluorescent dye layer was applied to the sample, a blank of that sample with only the glass and PS layer was taken. This method provided spectra with good baselines and low S/N. Steady-state fluorescence measurements were made on a Horiba (JobinYvon) Fluorolog spectrometer and collected at an angle of $60^{\circ}$. A xenon arc lamp excitation source at a wavelength of $520 \mathrm{~nm}$ was used to irradiate the samples. The emission range was set to $\lambda_{\max }-800 \mathrm{~nm}$ with slit widths of $3.0 \mathrm{~nm}$ for both the light source and detector. Excitation spectra were collected on the same instrument. The excitation range consisted of two scans, one from $350 \mathrm{~nm}$ to $\left(\lambda_{\max }-10 \mathrm{~nm}\right)$ and a second from $\left(\lambda_{\max }+10 \mathrm{~nm}\right)$ to $750 \mathrm{~nm}$ while monitoring fluorescence at a fixed wavelength.

\section{Result and Discussion}

To investigate the state of the dyes on the polystyrene surface, the UV/Vis absorbance spectrum was recorded as a function of fluorophore casting concentration and thus the film thickness for each dye, which can be seen in figure 2. The deconvoluted spectra for all dyes can be found in figures S1-S3 and the deconvolution parameters for the absorbance spectra of all dyes can be found in table S1. 

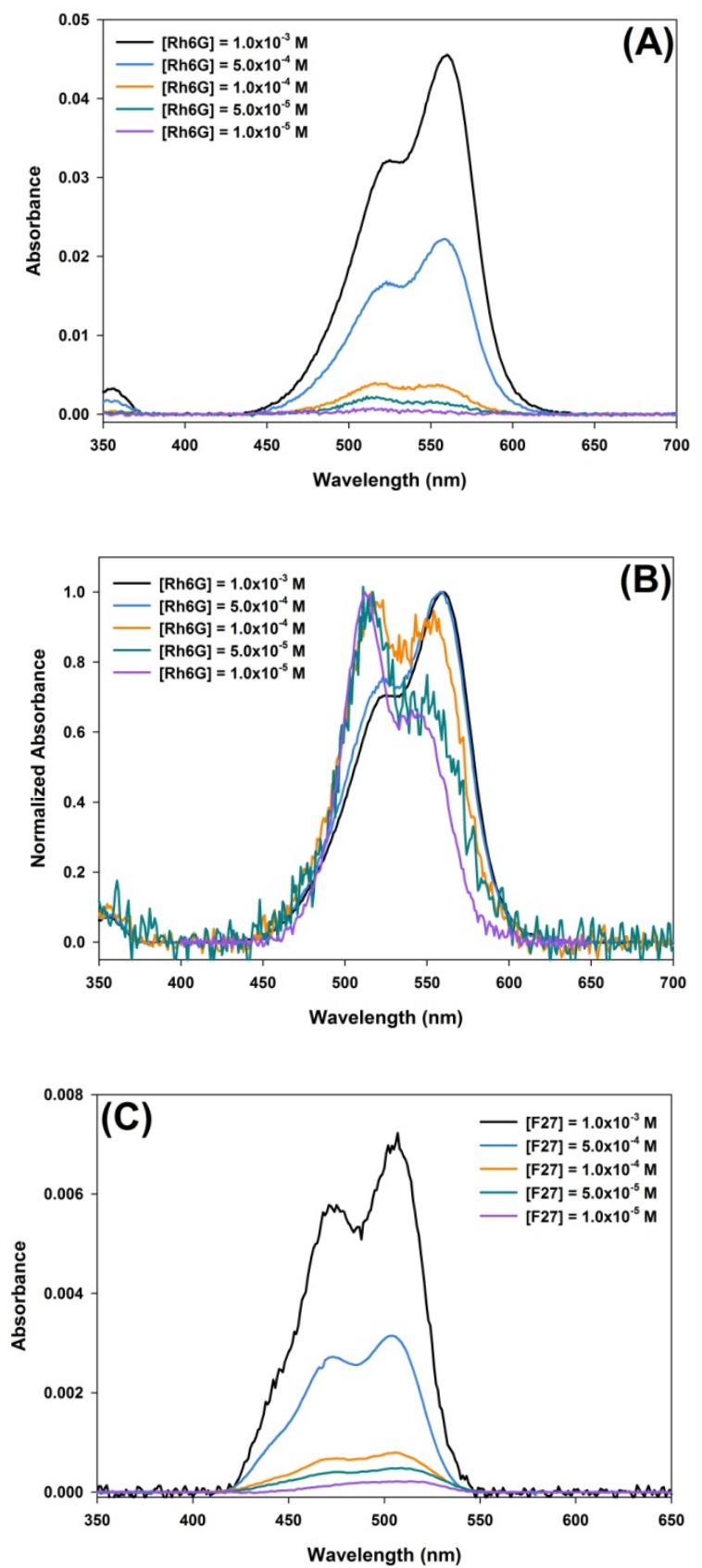

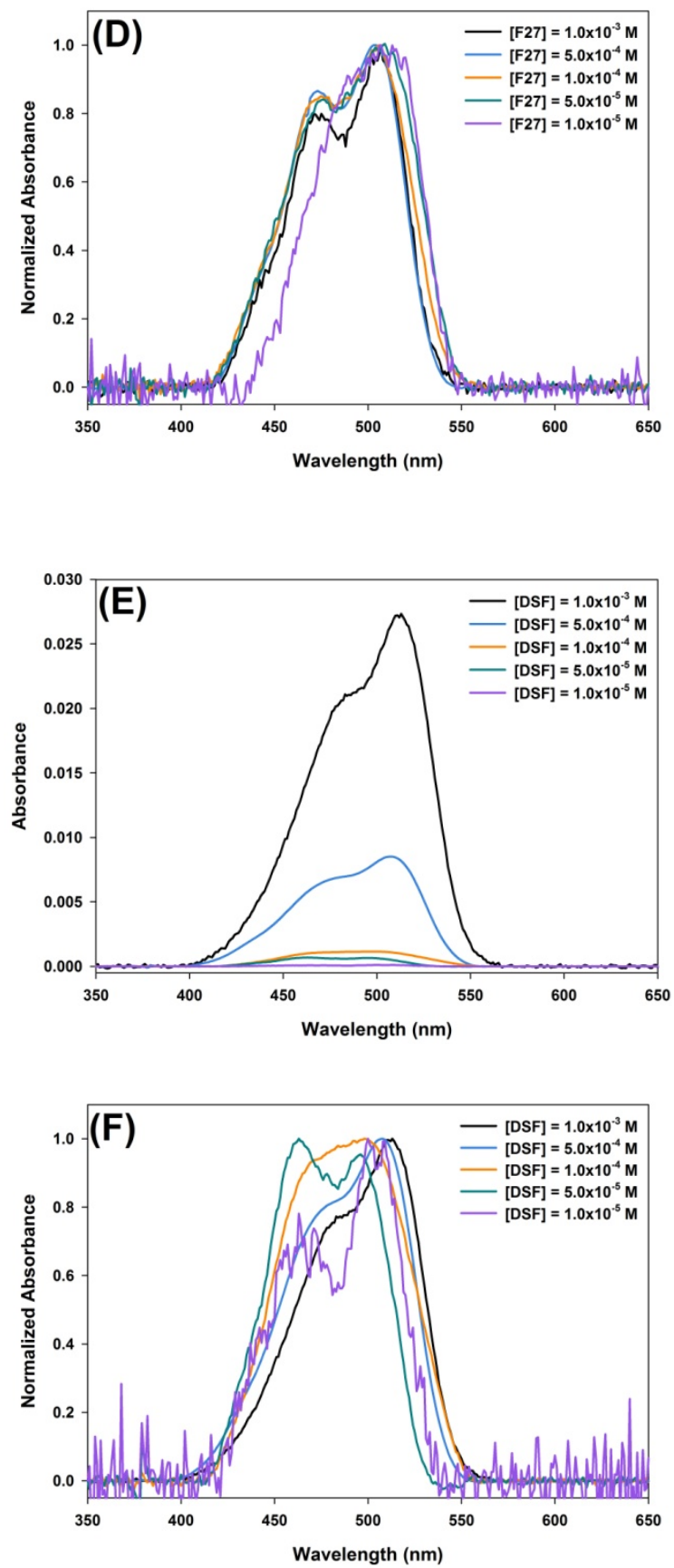

Figure 2. Absorbance spectra of A) Rh6G, C) F27, and E) DSF as a function of casting concentration. B, D, and F, are height normalized version of the same spectra, respectively.

The absorbance spectra reveal that there are some complicated dynamics occurring as the film forms on the PS surface. For all three dyes and for all casting concentrations 
there are multiple absorption peaks indicating either interactions between multiple dye molecules or dye/polymer interactions.
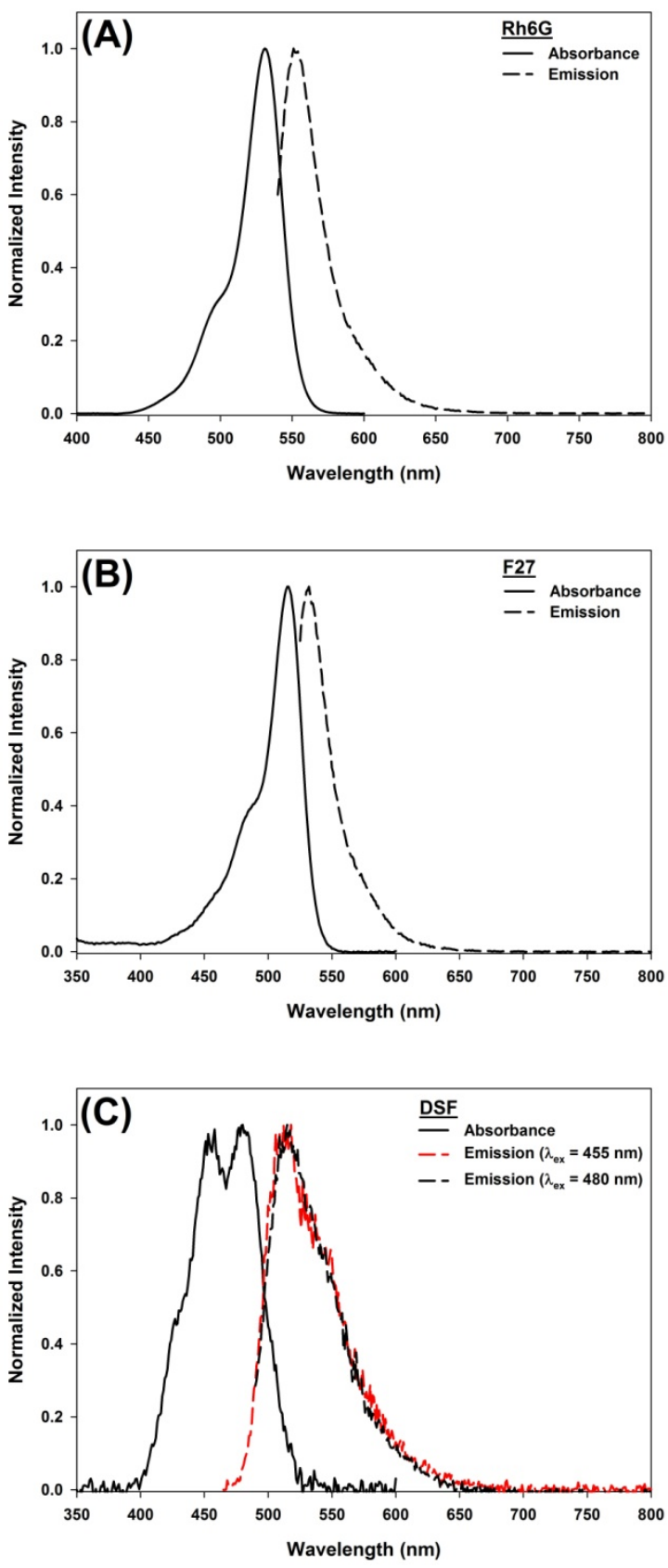

Figure 3. Dilute $\left(10^{-6} \mathrm{M}\right)$ absorbance and emission spectra of A) Rh6G, B) F27, and C) DSF dissolved in EtOH. 
To try and differentiate whether the additional absorption peaks that result from the thin films of the fluorophores was due to dye/dye or dye/polymer interactions, the dilute solution (EtOH) absorption and emission spectrum of each dye was collected. The spectral profiles can be seen in figure 3 and the deconvoluted spectra in figures S7-S9.

When comparing the lowest casting concentration of each dye to their respective dilute solution spectra, DSF is the only dye that looks somewhat similar. In solution, DSF has been shown to form a lactone ring between the $\mathrm{O}^{-}$on the carboxylic acid and the middle aromatic ring in the xanthene plane. ${ }^{19}$ This causes the absorption peak at $455 \mathrm{~nm}$ in solution while the other peak at $480 \mathrm{~nm}$ is the native form of the dye where no lactone is formed. In figure $3 \mathrm{C}$ the emission is shown at excitation wavelengths for both the lactone species and the native species of DSF. Since they completely overlap, this suggests that emission only comes from the native species and not the lactone. Deconvolution of the lowest casting concentration of DSF showed peaks at wavelengths corresponding to both the lactone and native species. This is a result of the lactone forming in solution before deposition.

For the case of Rh6G and F27, the lowest casting concentration for the thin films results in a markedly different absorbance spectrum when compared to the dilute solution spectra. The solution spectra for both dyes have a main narrow absorption peak that is associated with a higher energy shoulder. However, when the dyes are cast onto the PS surface their spectra broaden appreciably in both directions. Figure 2B shows the height normalized absorption spectra of Rh6G which demonstrates the biggest changes in the shape of the absorption curve as the film thickness increases. 
Deconvolution of the dilute solution spectrum of Rh6G showed two peaks, the main absorption at $532 \mathrm{~nm}$ and shoulder at $505 \mathrm{~nm}$. Comparatively, deconvolution of the thinnest Rh6G film required three peaks to fit. The main absorption peak and shoulder from the dilute spectrum shift $20 \mathrm{~nm}$ each to higher energies with the peaks being centered at $511 \mathrm{~nm}$ and $480 \mathrm{~nm}$. On an energy scale, these peaks are shifted by 772 $\mathrm{cm}^{-1}$ and $1031 \mathrm{~cm}^{-1}$, respectively, which makes sense as the PS surface is much less polar than EtOH. Furthermore, the deconvolution showed a third absorbing species that is centered at $548 \mathrm{~nm}$.

When the dilute solution of F27 was deconvoluted it required two peaks, one centered at $516 \mathrm{~nm}$ and a shoulder at $491 \mathrm{~nm}$, both of which are narrow and well defined. However, when F27 is cast as a thin film on the PS surface its absorption spectrum loses definition and the deconvolution of the spectrum for the thinnest film required three peaks to fit. Like the Rh6G, the peaks from the dilute spectrum shift $\sim 15 \mathrm{~nm}$ to higher wavelengths and are centered at $500 \mathrm{~nm}$ and $480 \mathrm{~nm}$, which correspond to energy shifts of $620 \mathrm{~cm}^{-1}$ and $467 \mathrm{~cm}^{-1}$, respectively. The third peak required to fit the thinnest film of F27 appears at $520 \mathrm{~nm}$.

Lastly, deconvolution of the thinnest DSF film required the use of four Gaussian peaks, which has one additional peak when compared to the dilute solution spectrum. The dilute solution spectrum consists of a small shoulder at $430 \mathrm{~nm}$ and two main absorption peaks at $451 \mathrm{~nm}$ and $479 \mathrm{~nm}$. Interestingly, DSF displays little to no spectral shift when cast into a thin film. The shoulder peak appears at $430 \mathrm{~nm}$ while two other peaks appear at $455 \mathrm{~nm}$ and $475 \mathrm{~nm}$. The fourth peak in the deconvolution of the thin film absorption spectrum appears at $505 \mathrm{~nm}$. The parameters for the 
deconvolution of the absorbance spectra for all casting concentrations and dyes can be seen in Table S1. As the film thickness increases the spectra for all dyes broaden and shift, which is most likely the result of dimerization and self-aggregation of the dyes, which xanthene dyes are prone to. ${ }^{20}$ However, the extent of aggregation can be used as another tool to probe ion- $\pi$ interactions.

When comparing the spectrum of the dilute solutions to the thinnest films for all dyes, the thin films required an additional peak, shifted into lower energy wavelengths with respect to the main absorption peak, for all dyes. It is important to note that the thinnest films are cast from dilute solutions that show no sign of higherorder aggregation before casting. Also due to the fact that surface coverage is low and neighboring dye molecules are spread out for the thinnest films, these additional absorption peaks are likely due to an interaction between a monomer dye molecule and the PS surface, but due to the surface morphology of the PS film there could be confined areas that trap dye molecules where exciton dimers can be formed. Regardless of whether the peak is a result of a dye/polymer or a dye/dye interaction, an indication of the strength of that interaction is the energy difference between the additional peak and the monomer absorption peak for each dye, which is summarized in Table 1.

Table 1. Comparison of interaction strength based on peak separation.

\begin{tabular}{|l|r|r|r|r|r|}
\hline Dye & $\begin{array}{c}\text { Monomer Peak } \\
(\mathbf{n m})\end{array}$ & $\begin{array}{c}\text { Low Energy Peak } \\
(\mathbf{n m})\end{array}$ & $\Delta \lambda(\mathbf{n m})$ & $\Delta E\left(\mathrm{~cm}^{-\mathbf{1}}\right)$ & $\begin{array}{c}\Delta E \\
(\mathbf{k c a l} / \mathbf{m o l})\end{array}$ \\
\hline $\mathrm{Rh}^{+} \mathrm{G}^{+}$ & 511 & 548 & 37 & -1321 & 15.8 \\
\hline $\mathrm{DSF}^{2-}$ & 475 & 505 & 30 & -1251 & 15.0 \\
\hline F27 & 500 & 520 & 20 & -769 & 9.2 \\
\hline
\end{tabular}


Due to the similar molecular structure of the three dyes used here, these shifts in energy can be understood by analyzing the subtle structural differences that occur from one dye to the next. The dye that experience the smallest shift in energy when cast onto the PS is F27. Possible interactions between F27 and the aromatic rich PS surface or another F27 molecule are limited because F27 is a neutral dye. Therefore, a complex formed between F27 and PS aromatic rings or two F27 molecules is a result of only $\pi-\pi$ interactions and very weak H-bonding, which results in the least stabilized complex. Furthermore, F27 has two Cl substituent groups both of which weakly deactivate the aromatic xanthene plane and can potentially have an effect on the $\pi-\pi$ interaction.

For the case of DSF, a much stronger shift is observed when cast onto the PS surface. Due to the fact that DSF is a divalent anion, the case for ion- $\pi$ interactions must be considered. Anion- $\pi$ interactions occur in systems where the aromatic component is experiencing electron withdrawing effects from substituent groups. Polystyrene consist of an alkyl backbone, which results in little to no inductive effect on the electron density on the aromatic ring. In fact, the only electron deficient aromatic ring in the system of DSF on PS is the phenyl ring coming off the xanthene plane in DSF. This phenyl ring has a carboxylic acid substituent which draws electron density away from the ring. However, this effect is negligible at this casting concentration for two reasons. For one, some of the DSF forms the lactone ring with the electron withdrawing carboxylic acid which changes the properties of that ring system, and two at this thickness neighboring dye molecules are spread out. Therefore, it is unlikely that any anion- $\pi$ interaction is contributing to the energy shift in the 
DSF/PS or DSF/DSF complex. This means that $\pi-\pi$ interaction and H-bonding are responsible for the DSF/PS or DSF/DSF complex, similar to the F27. The difference between DSF and F27 is the magnitude of the shift and this can be explained by the substituent groups on the two dyes. DSF has an $\mathrm{O}^{-}$bonded to the xanthene plane which is a very strong electron donating group. Because of this, the $\pi$ system in the xanthene plane is more activated which could lead to a stronger $\pi-\pi$ interaction. Additionally, the $\mathrm{O}^{-}$may partake in $\mathrm{H}$-bonding, which can help stabilize a complex. Lastly, the largest energy shift observed belongs to the complex formed by Rh6G and PS. This is expected because not only can Rh6G interact in all the ways that the previous dyes can in terms of $\pi-\pi$ interactions and H-bonding, but it also has the potential to form cation- $\pi$ bonds with the positively charged ethylamine on the Rh6G and the aromatic ring on the PS surface or the xanthene plane on another Rh6G molecule.

Additional evidence for the potential of cation- $\pi$ interactions comes from examination of the emission spectra. Like the absorbance spectra, the emission was recorded as a function of casting concentration, and effectively film thickness for each dye. The emission spectra can be seen in figure 4 and the deconvoluted spectra in figure S4-S6. The deconvolution parameters for the emission spectra of all dyes can be found in table S1. 

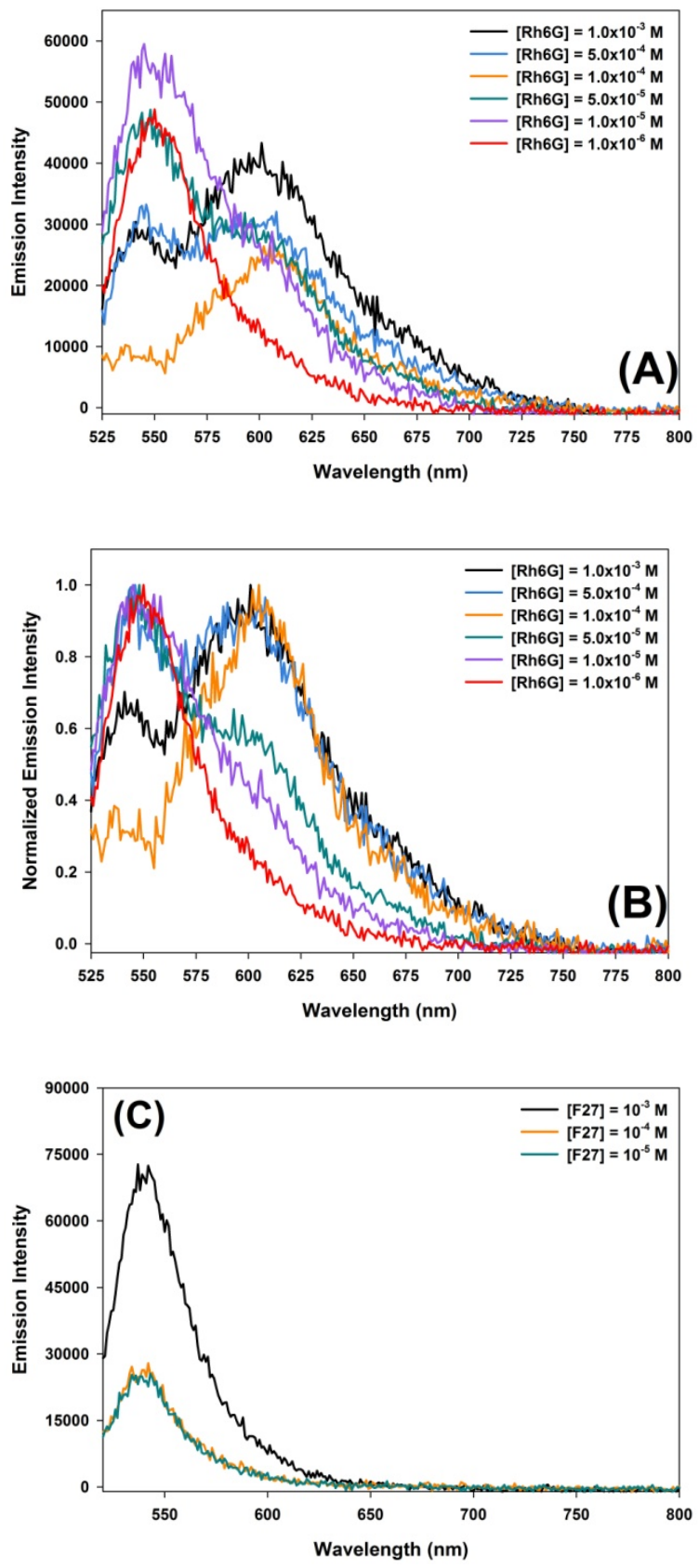

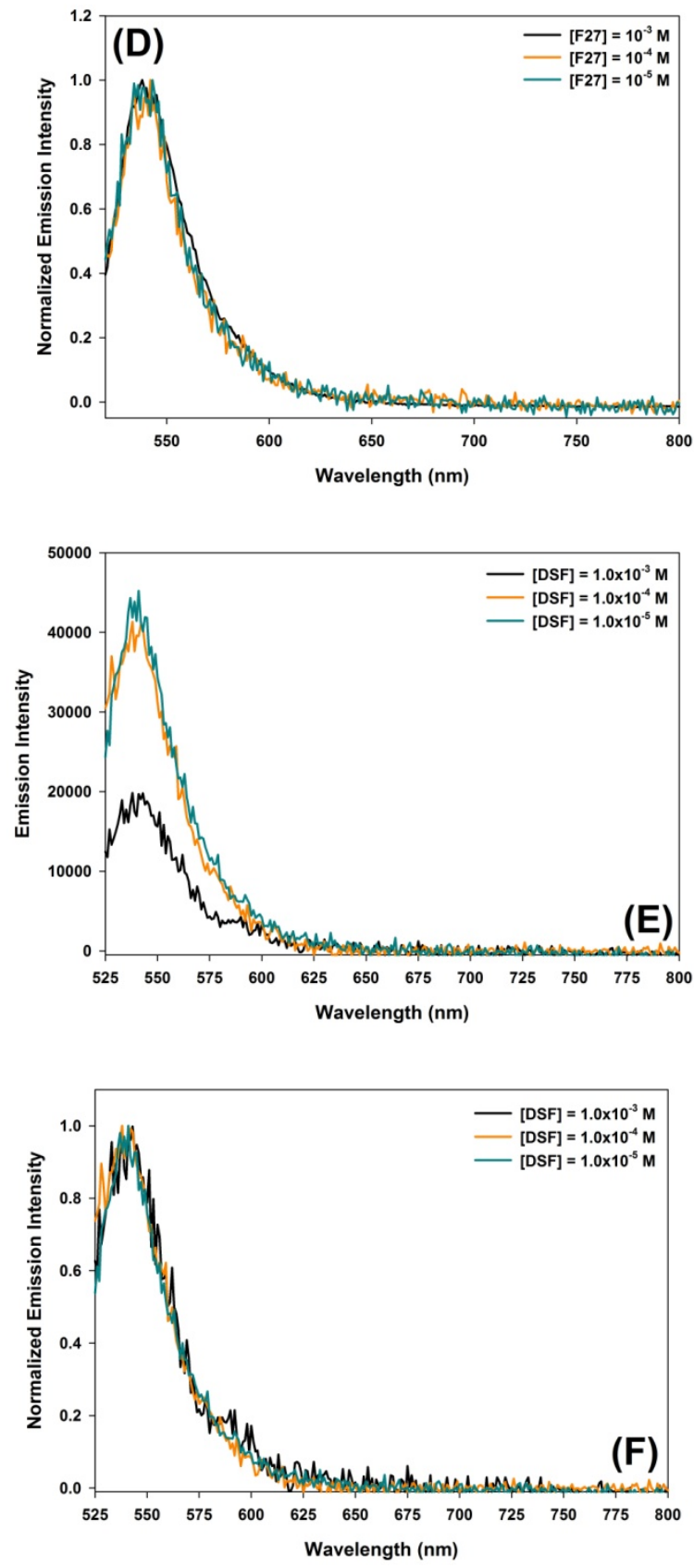

Figure 4. Emission spectra and height normalized emission spectra as a function of casting concentration of A) \& B) Rh6G, C) \& D) F27, and E) \& F) DSF.

Comparing the emission of the dyes in the thin film form to the dilute solution, there are some drastic changes. Specifically, the spectra for Rh6G and DSF change in shape the most, with Rh6G gaining more emission peaks and DSF losing some. For Rh6G, 
the emission in EtOH consists of a narrow, intense peak centered at $551 \mathrm{~nm}$ with a small shoulder at $567 \mathrm{~nm}$, similar to the absorbance spectrum. In contrast, when Rh6G is cast as a film the emission spectrum broadens and multiple peaks arise. Deconvolution of the Rh6G thin films revealed three emission peaks for all thicknesses, one at $560 \mathrm{~nm}$, another at $608 \mathrm{~nm}$, and lastly a peak at $648 \mathrm{~nm}$.

What is more telling about the interactions on the surface of the PS is the intensity of the emission spectra at the lowest casting concentration. In the absorbance spectrum for $[\mathrm{Rh} 6 \mathrm{G}]=1.0 \times 10^{-5} \mathrm{M}$, there are multiple peaks but it is dominated by the monomer absorption and as the film thickness grows more aggregation occurs. By this logic, the most intense emission spectrum of the Rh6G films should correspond to the lowest casting concentration, but this is not the case. The emission spectrum for $[\mathrm{Rh} 6 \mathrm{G}]=1.0 \times 10^{-6} \mathrm{M}$ shows primarily monomer emission but the intensity is less than the spectrum for $[\mathrm{Rh} 6 \mathrm{G}]=1.0 \times 10^{-5} \mathrm{M}$. This means that monomer emission is being quenched by some source, and the most likely option is that a weakly emissive exciplex is formed between excited Rh6G molecules and ground state PS, the emission of which overlaps with the emission from an Rh6G excimer. This explains why the peak at $608 \mathrm{~nm}$ is present in the emission spectra of films cast from very dilute Rh6G solutions. At low concentrations it results from the weak emission of the Rh6G/PS exciplex and as the film thickness increases the intensity of the peak increases due to Rh6G/Rh6G excimer formation. Additional evidence of an exciplex comes from the examination of the fluorescence lifetimes of Rh6G on the PS surface. Decay curves of various casting concentrations of for all dyes on PS are shown in figure 5 and the lifetime values are summarized in table 2. The decays are collected at 
$550 \mathrm{~nm}$ for Rh6G and $540 \mathrm{~nm}$ for both DSF and F27. The black curve corresponds to the instrumental response function.
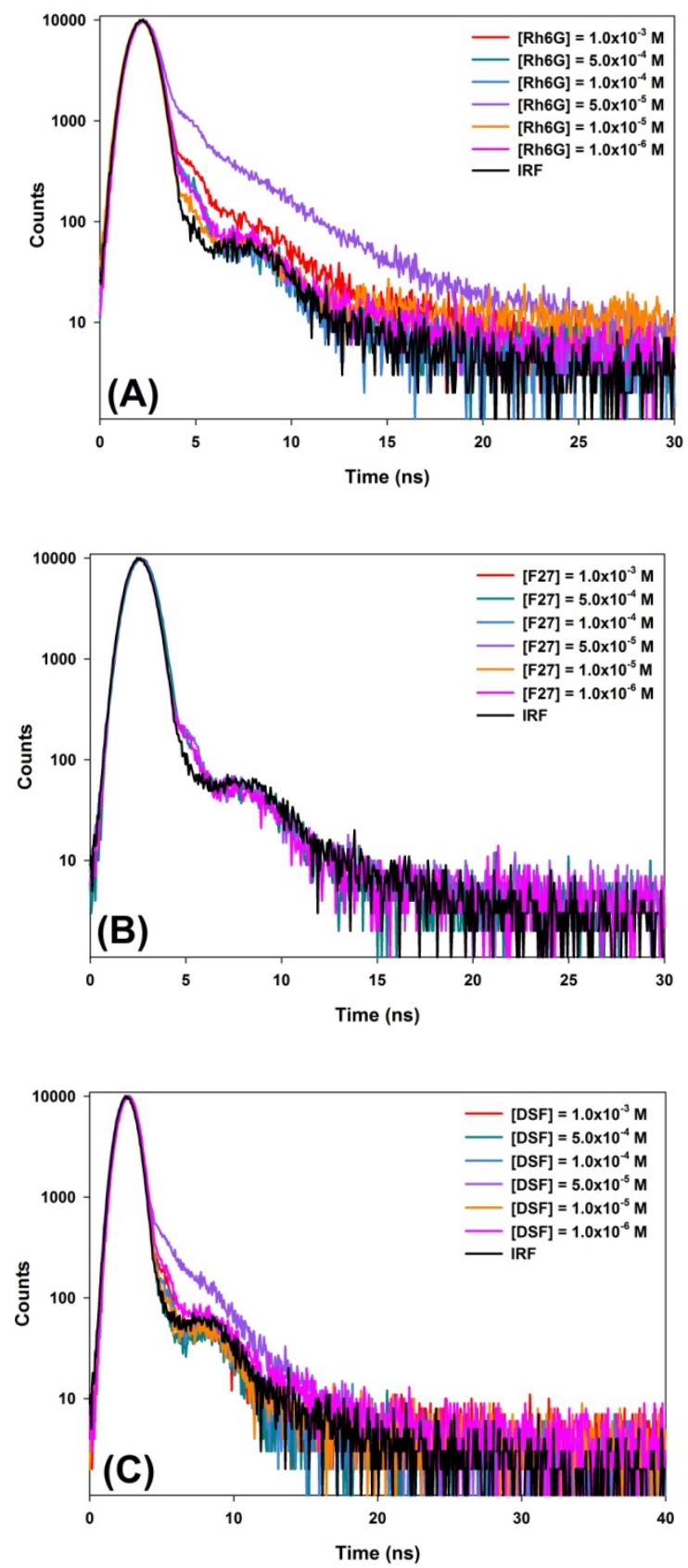

Figure 5. Fluorescence lifetime decays of various casting concentrations of A) Rh6G $\left(\lambda_{\mathrm{em}}=550 \mathrm{~nm}\right)$, B) F27 $\left(\lambda_{\mathrm{em}}=540 \mathrm{~nm}\right)$, and C) DSF $\left(\lambda_{\mathrm{em}}=540 \mathrm{~nm}\right) \cdot \lambda_{\mathrm{ex}}=452 \mathrm{~nm}$. 
Table 2. Lifetimes and component contribution from decay fits. $\alpha_{\mathrm{i}}$ is the preexponential factor that shows the relative percentage that the $i^{\text {th }}$ component contributes to the overall decay. $\tau_{\mathrm{i}}$ represents the average lifetime of the $i^{\text {th }}$ component. $\chi^{2}$ is representative of how good the exponential fit is and should be as close to 1 as possible.

\begin{tabular}{|c|r|r|r|r|r|r|r|r|}
\hline Dye & Concentration & \multicolumn{1}{|c|}{$\boldsymbol{\alpha}_{1}$} & $\boldsymbol{\tau}_{1}(\mathbf{n s})$ & $\boldsymbol{\alpha}_{2}$ & $\boldsymbol{\tau}_{\mathbf{2}}(\mathbf{n s})$ & $\boldsymbol{\alpha}_{3}$ & $\boldsymbol{\tau}_{3}(\mathbf{n s})$ & $\chi^{2}$ \\
\hline \multirow{3}{*}{$\mathrm{Rh}^{+} \mathrm{G}^{+}$} & $1.0 \times 10^{-3} \mathrm{M}$ & 0.93 & 0.06 & 0.05 & 1.4 & 0.02 & 4.8 & 1.77 \\
\cline { 2 - 9 } & $5.0 \times 10^{-5} \mathrm{M}$ & 0.77 & 0.06 & 0.12 & 1.5 & 0.11 & 4.2 & 1.53 \\
\cline { 2 - 9 } & $1.0 \times 10^{-5} \mathrm{M}$ & 0.97 & 0.05 & 0.03 & 3.6 & N/A & N/A & 1.44 \\
\hline $\mathrm{DSF}^{2-}$ & $5.0 \times 10^{-5} \mathrm{M}$ & 0.89 & 0.11 & 0.07 & 1.6 & 0.04 & 4.1 & 1.22 \\
\hline
\end{tabular}

Looking at the film cast from $[\mathrm{Rh} 6 \mathrm{G}]=1.0 \times 10^{-5} \mathrm{M}$, there are two exponential functions in the fit, a short lifetime of $\tau_{1}=0.05 \mathrm{~ns}$ and a longer lifetime of $\tau_{2}=3.6 \mathrm{~ns}$. Although there are two lifetimes, the decay is dominated by the shorter lifetime which constitutes $97 \%$ of the overall decay. There have been many studies that have measured the lifetime of Rh6G in solution with various different solvents and it is found that the lifetime of monomeric Rh6G ranges from 3.8 - 4.4 ns, depending on the polarity of the solvent. ${ }^{21-23}$ Additionally, in a previous report we measured the lifetime of solid phase Rh6G on a poly(vinylidene fluoride) surface as a function of film thickness and it was found the monomeric Rh6G had a lifetime of $3.9 \pm 0.3$ ns. $^{24}$ This implies that the longer lifetime measured for the film cast from $[\mathrm{Rh} 6 \mathrm{G}]=1.0 \times 10^{-5} \mathrm{M}$, $\tau_{2}=3.1$ ns, corresponds to the emissive monomeric Rh6G on the PS surface. Looking at the absorbance spectrum of the film cast from $[\mathrm{Rh} 6 \mathrm{G}]=1.0 \times 10^{-5} \mathrm{M}$ (Figure $2 \mathrm{~A}$ and 2B), the main absorption peak is at $511 \mathrm{~nm}$ with a small shoulder at $480 \mathrm{~nm}$ and a lower energy peak at $548 \mathrm{~nm}$. This suggest that the surface is covered primarily by monomeric Rh6G which is also supported by the emission spectrum for [Rh6G] = $1.0 \times 10^{-5} \mathrm{M}$, as the monomer peak is the most intense in that spectrum as well. Considering these spectra along with the data from the lifetime decay, it indicates that 
the short lifetime component, $\tau_{1}=0.05 \mathrm{~ns}$, must be originating from the monomer Rh6G interacting with another molecule. As the casting concentration increase the decays require three exponentials to fit which is due to the excimer, $\tau_{3}=4.2 \mathrm{~ns}$, forming in concentrations large enough to be detected by this method.

It is interesting to note that only three casting concentrations of Rh6G and one casting concentration of the DSF were able to be fit with $\chi^{2}<2$. This is due to the fact that the dynamics of absorption and emission of the dyes on the surface of the PS are a complicated process. However, for both the charged dyes, Rh6G and DSF, the decays from films cast from a $5.0 \times 10^{-5} \mathrm{M}$ solution give very similar results. Like the Rh6G, the lifetime of the DSF is dominated by a very fast decaying component, $\tau_{1}=0.11 \mathrm{~ns}$, which constitutes $89 \%$ of the decay and is accompanied by two other slower fluorescing components $\tau_{2}=1.6$ ns and $\tau_{3}=4.1 \mathrm{~ns}$, which correspond to the monomer and excimer DSF, respectively. This matches well with steady-state emission spectra for the films of DSF which when deconvoluted all casting concentrations required two peaks, one for the monomer emission and another for the exciplex/excimer. The trend in the intensity for the DSF is similar to that of Rh6G. Considering only aggregation effects, the lowest casting concentration should have the highest emission, but the emission for both the films cast from $1.0 \times 10^{-5} \mathrm{M}$ and $1.0 \times 10^{-4} \mathrm{M}$ are equal, indicating that monomer emission of DSF from the $1.0 \times 10^{-5} \mathrm{M}$ film is being quenched by some source.

For the case of F27 no decay could be fit to give a $\chi^{2}<2$, and therefore no information about the excited state lifetime could be obtained. This likely originates from the fact that the F27 films give absorbance intensities that are an order of 
magnitude less than the films of Rh6G and DSF, which leads to low emission that could not be detected. However, looking at the steady-state spectra for F27 in figure 4C, it is clear that the intensity trend is different from that of Rh6G and DSF. Instead of decreasing emission intensity as the casting concentration increases, F27 displays the opposite behavior where the film cast from $1.0 \times 10^{-3} \mathrm{M}$ has the highest intensity. From an aggregation perspective this would suggest that F27 forms emissive aggregates, however looking at the normalized emission spectra in figure 4D, it is clear that the shape of the spectrum does not change as the casting concentration increase. This shows that aggregation is not what is responsible for the increase in emission. Deconvolution of the emission spectra show two peaks for all concentrations. Considering all the data, it implies that F27 still has complicated dynamics on the PS surface, but the monomers are not affected by an interaction with the PS itself, instead they free to emit and as the film thickness increases the emission grows from both the monomer and eventually the formation of excimers.

The emission data for all dyes suggest that there are interactions occurring for the charged dyes that do not happen with a neutral dye. For both Rh6G and DSF, the phenomena of aggregation was mentioned, however it is unlikely that the short lifetime originates from the aggregated species due to the excitation wavelength of the laser used to record the decays. The laser emits $452 \mathrm{~nm}$ light and in that wavelength region the aggregated species of both dyes has little to no absorption and by effect no emission either. Furthermore, there is very little aggregate formed on the surface at this low of a casting concentration. Excimers have been shown to have longer lifetimes than their respective monomer components due to a mismatch in franck- 
condon states, which rules out that interaction being assigned to the short lifetime. ${ }^{25,26}$ Therefore, by process of elimination the only other source of interaction for a monomer DSF or Rh6G molecule must be with the PS surface itself, which is produced by the coordination from ion- $\pi$ interactions.

\section{Conclusion}

This report investigates the potential for ion- $\pi$ interactions between charged organic dyes by comparing the spectral response of a cationic dye (Rh6G), an anionic dye (DSF), and a neutral dye (F27) on the surface of a polystyrene film. Absorbance spectroscopy was used to investigate the state of the dyes on the PS surface. It was found for all the dyes that a lower energy absorbing species is formed at the lowest casting concentration, which is attributed to either dye/PS or dye/dye interactions. The interaction energy of the dye complex, whether it is from an interaction between the dye and PS or two dye molecules, is proportional to the spectral shift of the aggregated peak from the monomer peak and can be used as an indication of ion- $\pi$ interactions. The spectral shifts, from most intense to least intense, follow the trend of: Rh6G > DSF $>$ F27. Due to the fact that all three dyes share similar chemical structure that includes a $\pi$ rich xanthene plane, the increase in interaction energy can be attributed to stabilization by ion- $\pi$ interactions. Furthermore, the use of fluorescence spectroscopy can be used as an additional tool to identify potential ion- $\pi$ interactions between the charged dyes and the $\pi$ rich PS surface. By analyzing the trends in emission intensity as a function of dye film casting concentration and correlating these trends with the excited state lifetimes it was found that a large portion of monomer emission from the 
charged dye films is quenched by the formation of a weakly emissive exciplex. No evidence of the exciplex is found for the F27, which suggest that ion- $\pi$ interactions are responsible for the formation of the exciplex between an excited Rh6G or DSF molecule and the PS surface.

\section{ASSOCIATED CONTENT}

\section{Supporting Information}

The Supporting Information is available free of charge on the ACS Publications website. Deconvoluted absorbance and emission spectra for the three different dyes.

\section{Notes}

The authors declare no competing financial interest.

\section{ACKNOWLEDGEMENT}

This material is based upon work supported by the U.S. Department of Homeland Security, Science and Technology Directorate, Office of University Programs, under Grant Award 2013-ST-061-ED0001. The views and conclusions contained in this document are those of the authors and should not be interpreted as necessarily representing the official policies, either expressed or implied, of the U.S. Department of Homeland Security. 


\section{REFERENCES}

1. Sunner, J.; Nishizawa, K.; Kerbale, P. Ion-Solvent Molecule Interaction in the Gas Phase. The Postassium Ion and Benzene. J. Phys. Chem., 1981, 85, 1814-1820.

2. Solimannejad, M.; Hosseini, S. M.; Zabardasti, A. A Computational Study of Interplay Between Hydride Bonding and Cation $-\pi$ Interactions: $\mathrm{H}-\mathrm{MgH} \cdots \mathrm{X} \cdots \mathrm{Y}$

Triads $\left(\mathrm{X}=\mathrm{Li}+, \mathrm{Na}+, \mathrm{Y}=\mathrm{C}_{2} \mathrm{H}_{2}, \mathrm{C}_{2} \mathrm{H}_{4}, \mathrm{C}_{6} \mathrm{H}_{6}\right)$ as Model Systems. Mol. Phys., 2017, $115,825-830$.

3. Fu, W.; Carr, P. J. J.; Lecours, M. J.; Burt, M.; Marta, R. A.; Steinmetz, V.; Fillion, E.; McMahona, T. B.; Hopkins, W. S. Intramolecular Cation- $\pi$ Interactions in Protonated Phenylalanine Derivatives. Phys. Chem. Chem. Phys., 2017, 19, 729 - 734.

4. Khan, H. M.; Graúffel, C.; Broer, R.; MacKerell, Jr., A. D.; Havenith, R. W. A.; Reuter, N. Improving the Force Field Description of Tyrosine-Choline Cation $-\pi$ Interactions: QM Investigation of Phenol-N(Me $)_{4}{ }^{+}$Interactions. J. Chem. Theory Comput., 2016, 12, $5585-5595$.

5. Ma, J. C.; Dougherty, D. A. The Cation- $\pi$ Interaction. Chem. Rev., 1997, 97, $1303-1324$.

6. Borozana, S. Z.; Dimitrijevic, B. P.; Stojanovic, S. D. Cation- $\pi$ Interactions in High Resolution Protein-RNA Complex Crystal Structures. Comput. Biol. Chem., 2013, 47, $105-112$.

7. Fujisawa, I. and Aoki, K. Glycine Betaine Recognition through Cation $-\pi$ Interactions in Crystal Structures of Glycine Betaine Complexes with C-Ethyl- 
pyrogallol[4]arene and C-Ethyl-resorcin[4]arene as Receptors. Crystals, 2013, 306 314.

8. Lu, Q.; Danner, E.; Waite, J. H.; Israelachvili, J. N.; Zeng, H.; Hwang, D. S. Adhesion of Mussel Foot Proteins to Different Substrate Surfaces. J. R. Soc., Interface, 2013, 10, 79, $759-770$.

9. Gebbie, M. A.; Wei, W.; Schrader, A. M.; Cristiani, T. R.; Dobbs, H. A.; Idso, M.; Chmelka, B. F.; Waite, J. H.; Israelachvili, J. N. Tuning Underwater Adhesion with Cation- $\pi$ Interactions. Nat. Chem., 2017, DOI:10.1038/nchem.2720

10. Giese, M.; Albrecht, M.; Rissanen, K. Anion- $\pi$ Interaction with Fluoroarenes. Chem. Rev., 2015, 115, 16, 8867 - 8895.

11. Lakowitz, J. R. Principles of Fluorescence Spectroscopy, $3^{\text {rd }}$ ed.; Springer: New York, 2006, 237 - 242.

12. Huixiao, Y.; Qinzhi, H.; Guochun, M.; Guofeng, C.; Minli, T.; Wenqin, Z. $\mathrm{Hg}^{2+}$ Selective Fluorescent Chemosensor Based on Cation- $\pi$ Interaction. Chem. Res. Chin. Univ., 2014, 30, 6, 910 - 914.

13. Kumar, J.; Bhattacharyya, P. K.; Das, D. K. New Duel Fluorescent “on-off”' and Colorimetric Sensor For Copper(II): Copper(II) Binds Through N Coordination and $\pi$-Cation Interaction to Sensor. Spectrochim. Acta, Part A, 2015, 138, 99 - 104.

14. Xiaa, Y.; Wanga, X.; Zhanga, Y.; Luoa, B. Computational Investigations Into New Fluorescence Quenching Process Induced by Complexation of Alkali Metal Ion. J. Phys. Org. Chem., 2012, 25, 778 - 786.

15. Chen, W.; Elfeky, S. A.; Nonne, Y.; Male, L.; Ahmed, K.; Amiable, C.; Axe, P.; Yamada, S.; James, T. D.; Bull, S. D.; Fossey, J. S. A Pyridinium Cation- $\pi$ 
Interaction Sensor for the Fluorescent Detection of Alkyl Halides. Chem. Commun., 2011, 47, $253-255$.

16. Prodi, L.; Bolletta, F.; Montalti, M.; Zaccheroni, N.; Casanti, A.; Sansone, F.; Ungaro, R. Photophysics of 1,3-alternate Calix[4]arene-Crowns and of Their Metal Ion Complexes: Evidence for Cation- $\pi$ Interactions in Solution. New J. Chem., 2000, 24, $155-158$.

17. Lu, Q.; Oh, D.X.; Lee, Y.; Jho, Y.; Hwang, D. S.; Zeng, H. Nanomechanics of Cation- $\pi$ Interactions in Aqueous Solution. Angew. Chem. Int. Ed., 2013, 52, 3944 3948.

18. Eynde, X. V.; Weng, L. T.; Bertrand, P. Influence of Tacticity on Polymer Surfaces Studied by ToF-SIMS. Surf. Interface Anal., 1997, 25, 41 - 45.

19. Zhang, G.; Yu, B.; Chen, W. Spectroscopic Study on Sodium Fluorescein in Ethanol Solution. Wuli Huaxue Xuebao, 1992, 8, 4, 505 - 509.

20. Valdes-Aguilera, O.; Neckers, D. C. Aggregation Phenomena in Xanthene Dyes. Acc. Chem. Res.1989, 22, $171-177$.

21. Fedoseeva, M.; Letrun, R.; Vauthey, E. Excited-State Dynamics of Rhodamine 6G in Aqueous Solution and at the Dodecane/Water Interface. J. Phys. Chem. B2014, 118, 5184-5193.

22. Kemnitz, K.; Yoshihara, K. Entropy-Driven Dimerization of Xanthene Dyes in Nonpolar Solution and Temperature-Dependent Fluorescence Decay of Dimers. J. Phys. Chem.1991, 95, 6095 - 6104. 
23. Magde, D.; Wong, R.; Seybold, P. G. Fluorescence Quantum Yields and Their Relation to Lifetimes of Rhodamine 6G and Fluorescein in Nine Solvents: Improved Absolute Standards for Quantum Yields Photochem.Photobiol.2002, 75, 327-334.

24. Mullen, M.; Euler, W. B. The Influence of Interfacial Effects on the Photophysics of Rhodamine 6G Thin Films on a Poly(vinylidene fluoride) Surface.

Langmuir, 2017, 33, 9, 2194 - 2204.

25. Penzkofer, A.; Lu, Y. Fluorescence Quenching of Rhodamine 6G in Methanol at High Concentration. Chem. Phys., 1986, 103, 399- 405.

26. Lim, J. M.; Kim, P.; Yoon, M.; Sung, J.; Dehm, V.; Chen, Z.; Würthner, F.;

Kim, D. Exciton Delocalization and Dynamics in Helical $\pi$-Stacks of Self-Assembled Perylene Bisimides. Chem. Sci., 2013, 4, 388-397. 


\title{
A Spectroscopic Study of Charged Xanthene Dyes on a Polystyrene Surface: An
}

\section{Investigation into Potential Ion- $\pi$ Interactions}

\author{
Matthew Mullen and William B. Euler* \\ Department of Chemistry
}

140 Flagg Road

Kingston, RI 02881

\section{Supporting Information}

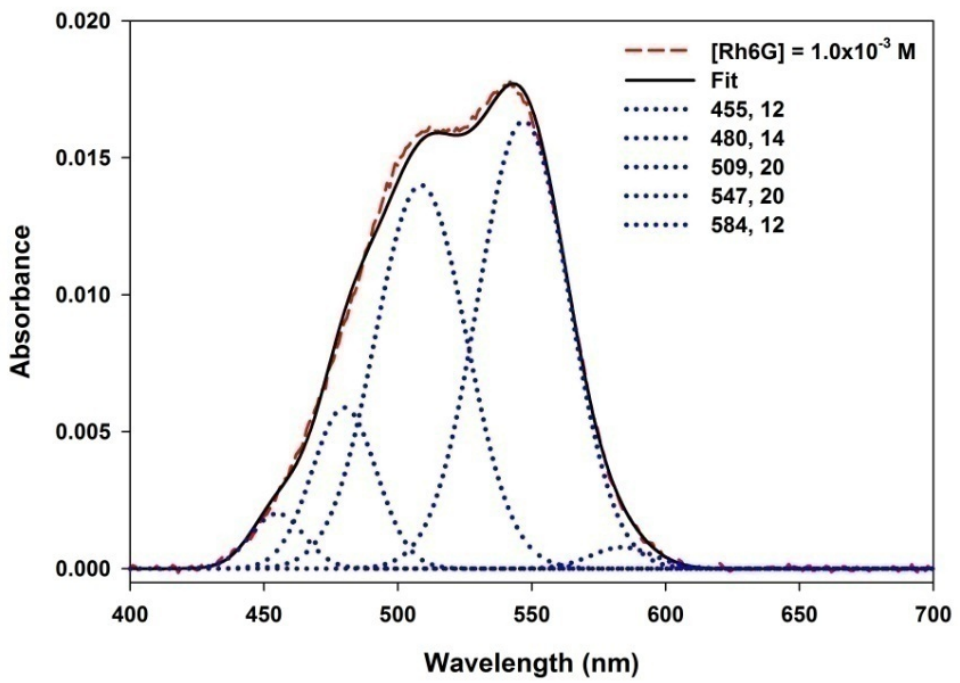



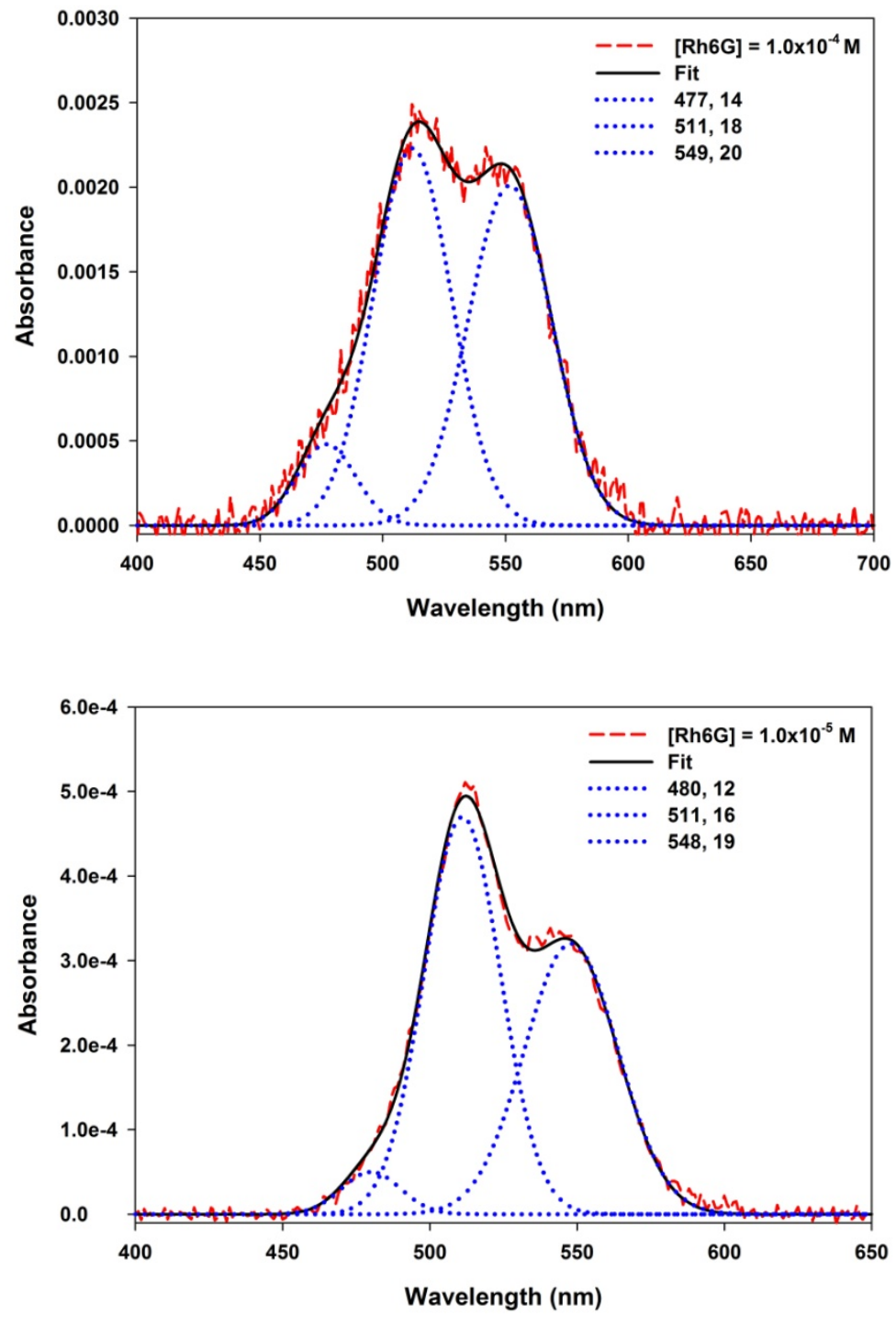

Figure S1. Deconvoluted absorbance spectra of films cast from different Rh6G concentrations. Legend shows peak position (nm) and full width at half max (nm). 

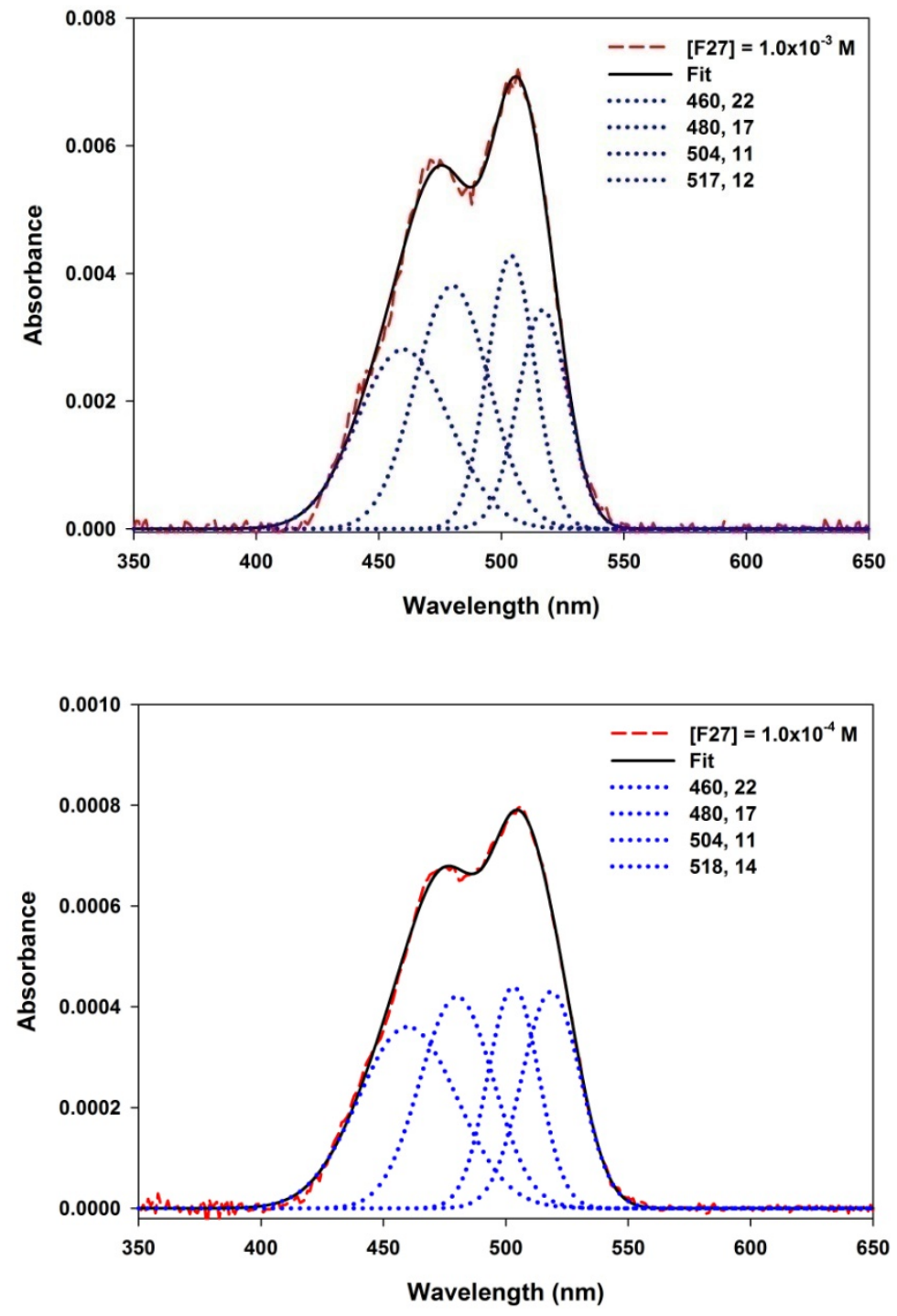


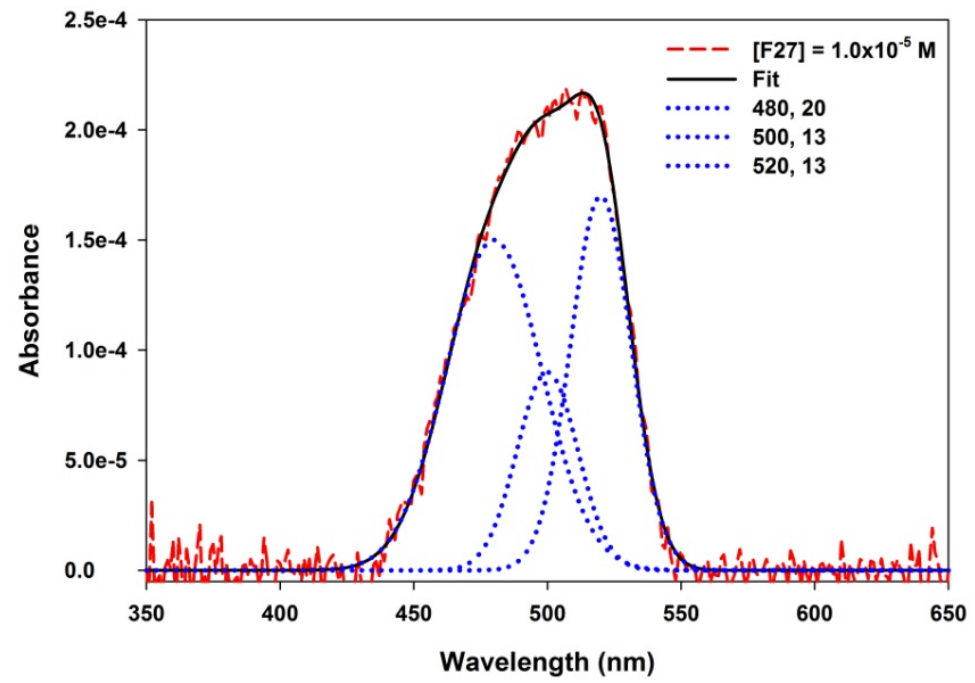

Figure S2. Deconvoluted absorbance spectra of films cast from different F27 concentrations. Legend shows peak position (nm) and full width at half max (nm).

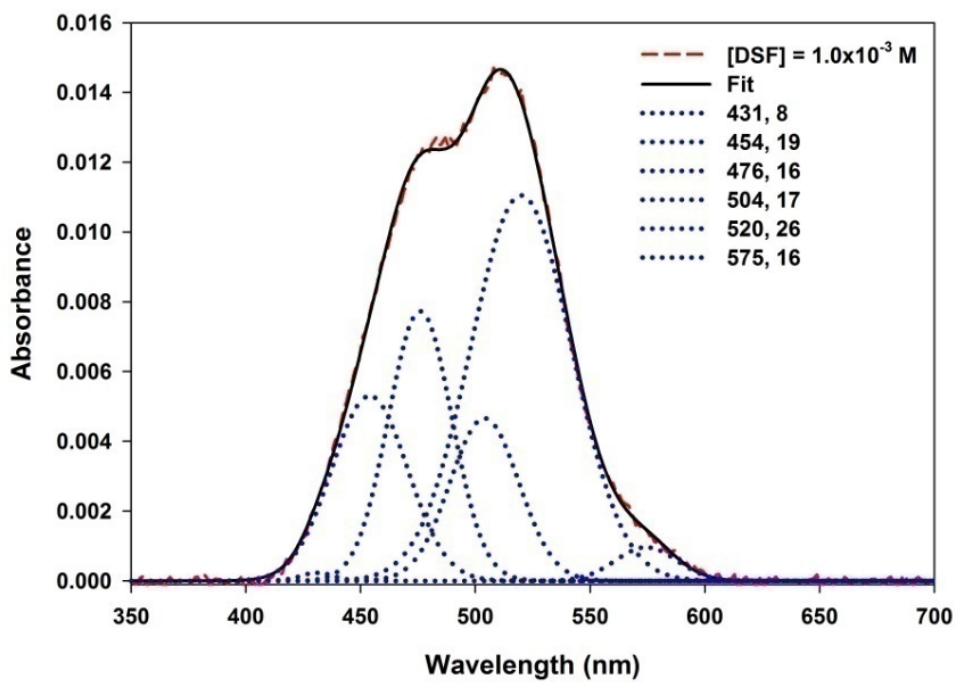



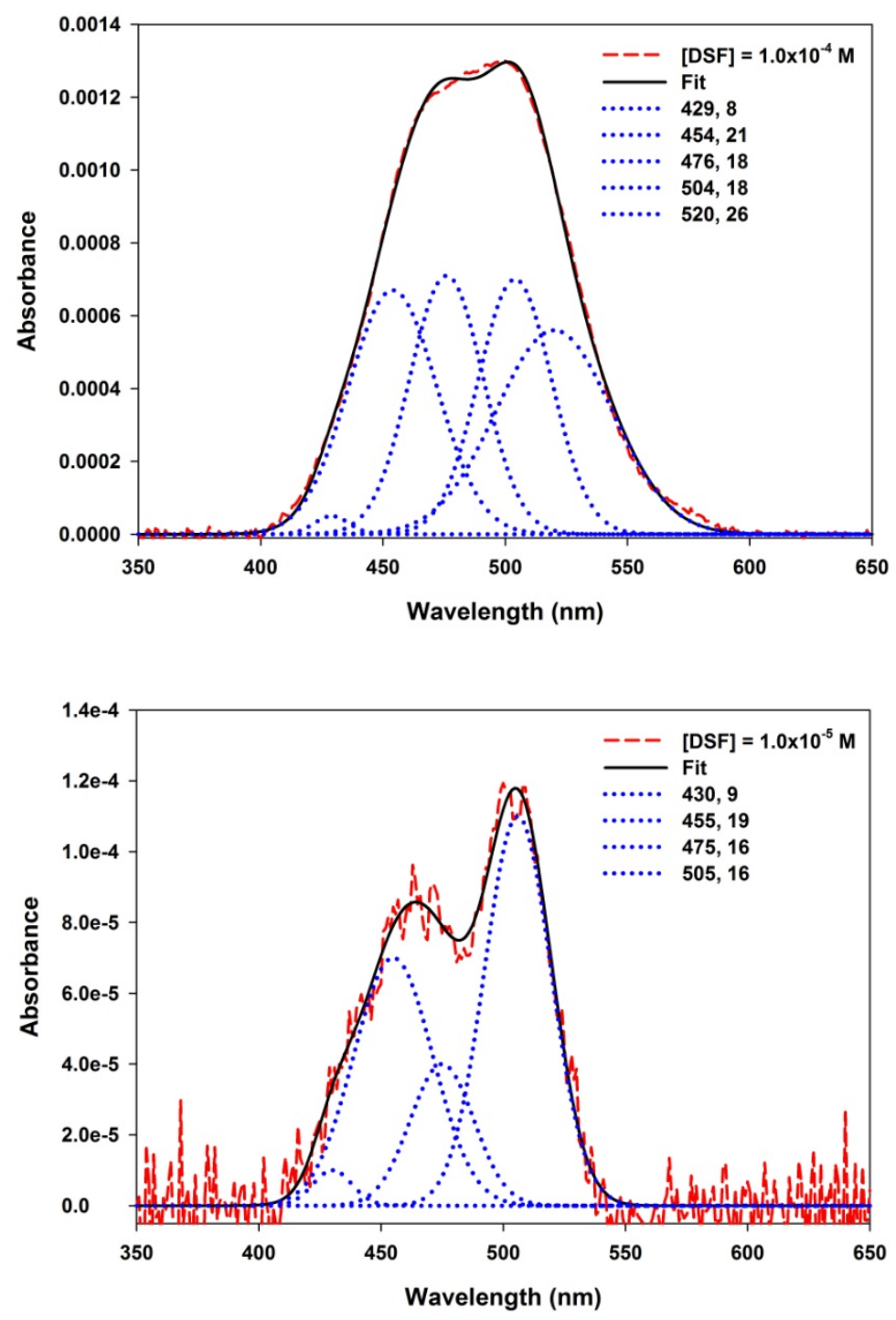

Figure S3. Deconvoluted absorbance spectra of films cast from different DSF concentrations. Legend shows peak position (nm) and full width at half max (nm). 

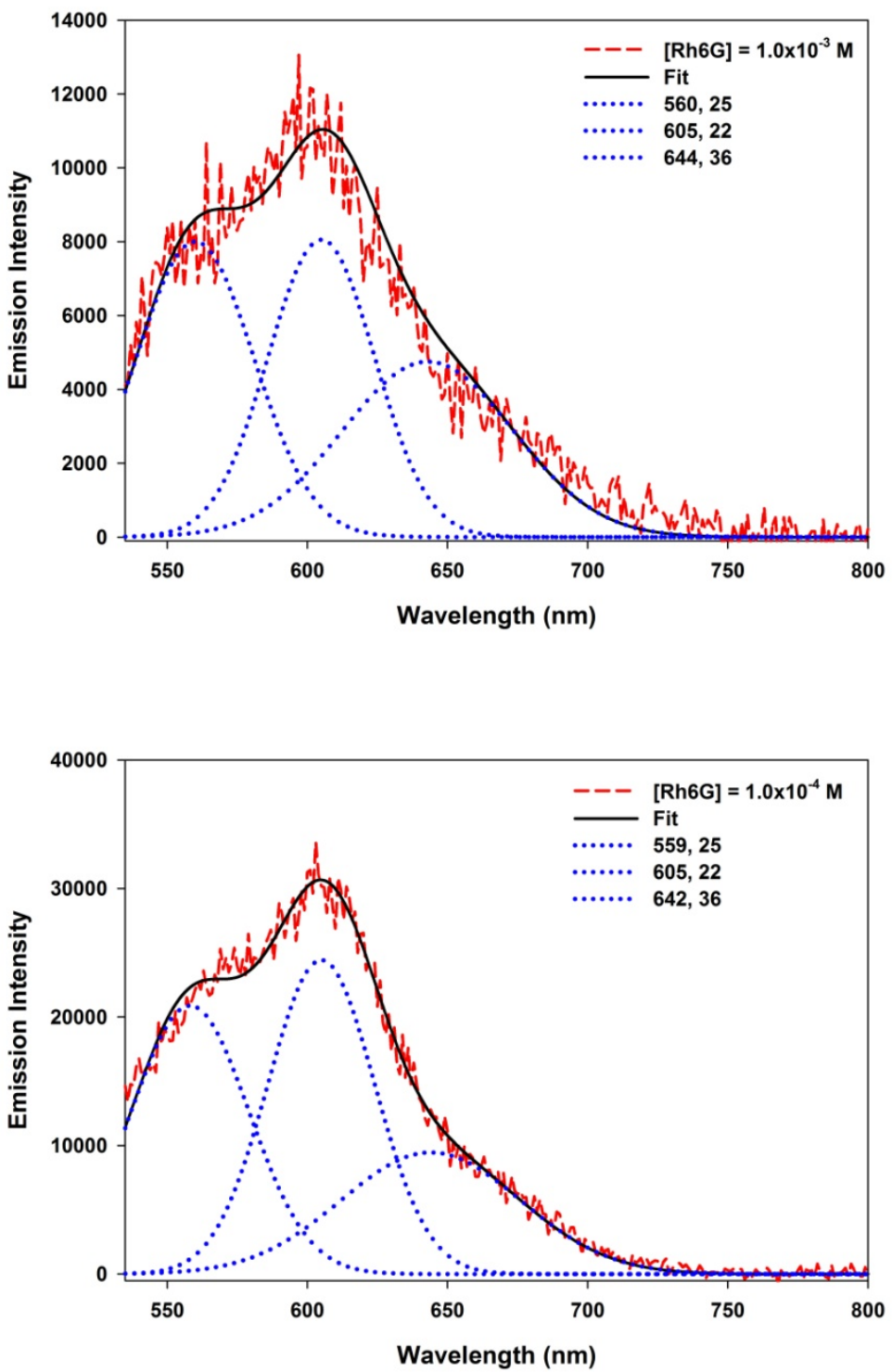


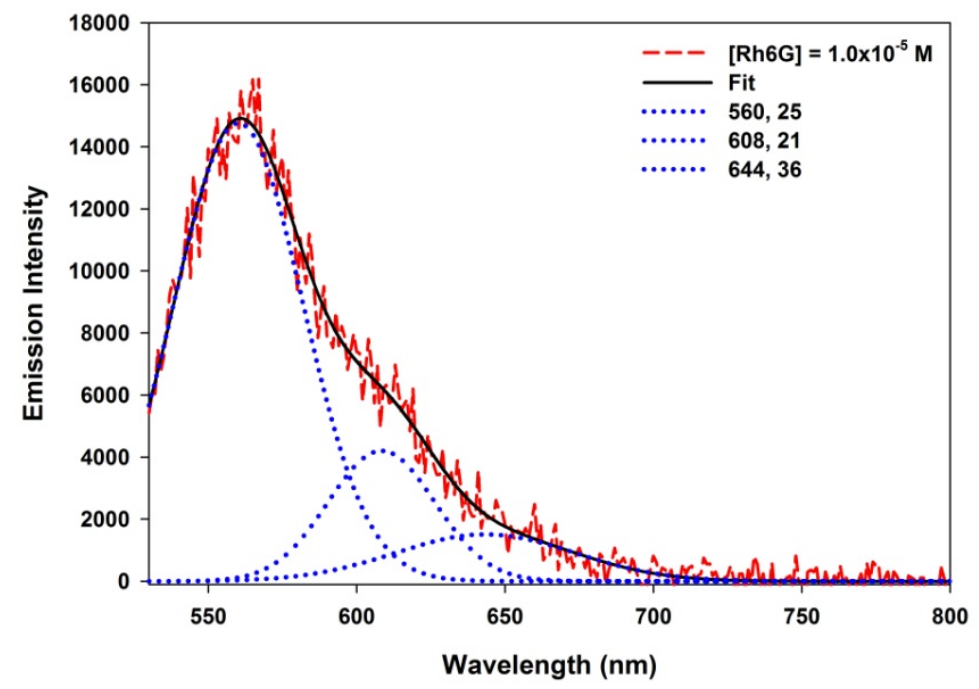

Figure S4. Deconvoluted emission spectra of films cast from different Rh6G concentrations. Legend shows peak position (nm) and full width at half max (nm).

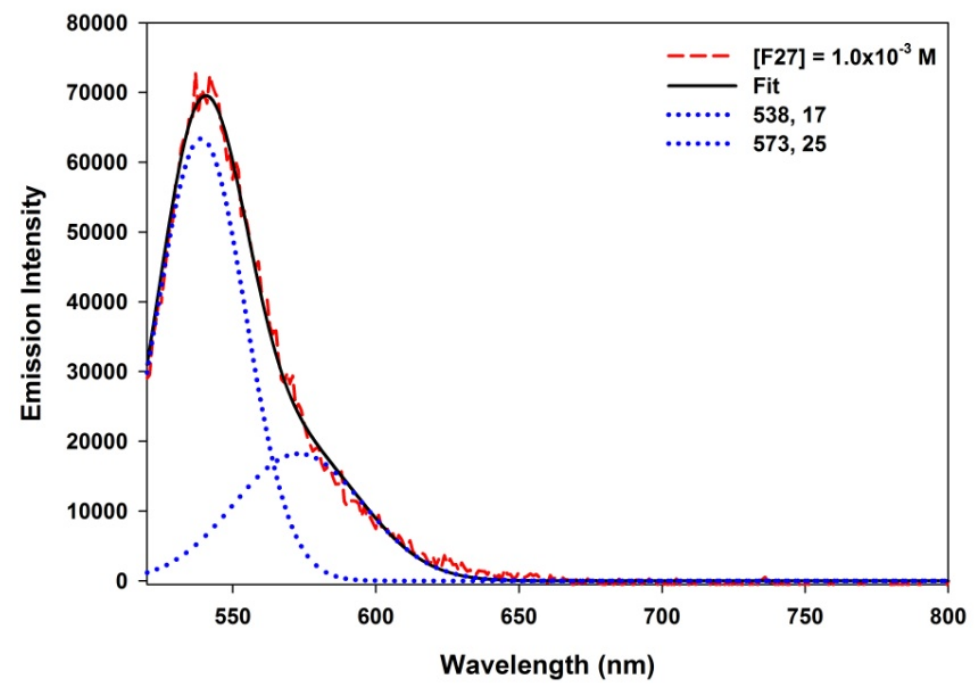



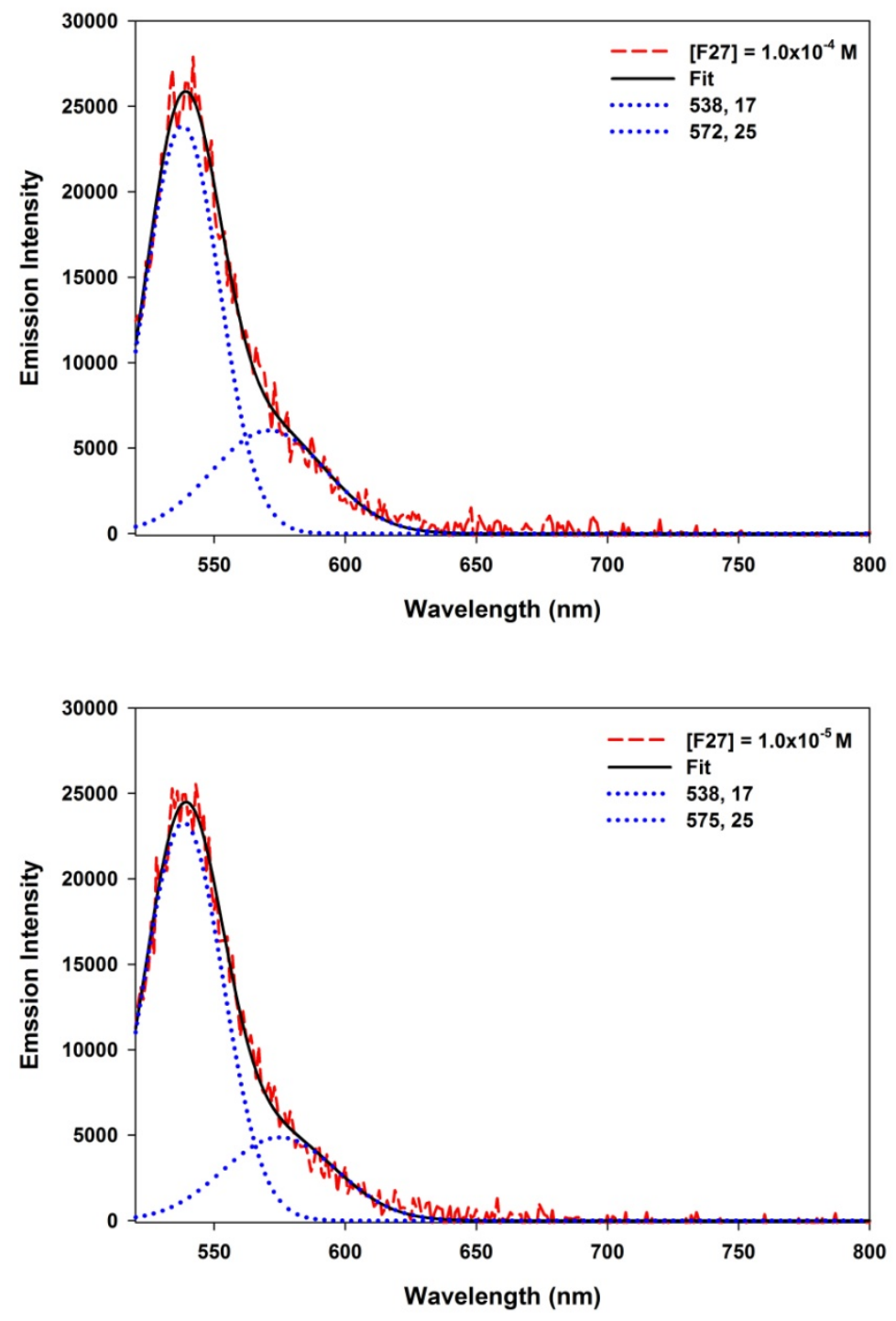

Figure S5. Deconvoluted emission spectra of films cast from different F27 concentrations. Legend shows peak position (nm) and full width at half max (nm). 

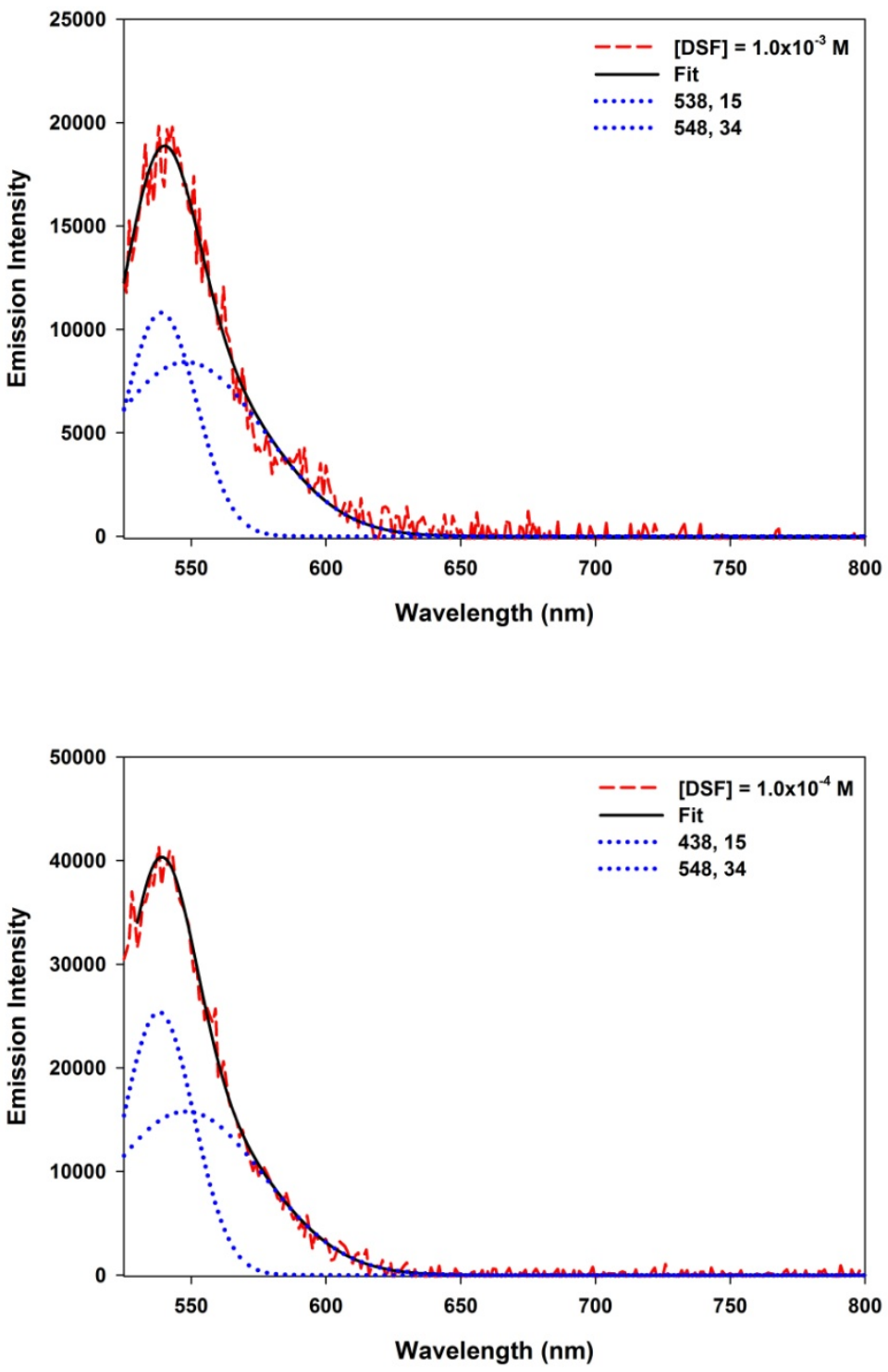


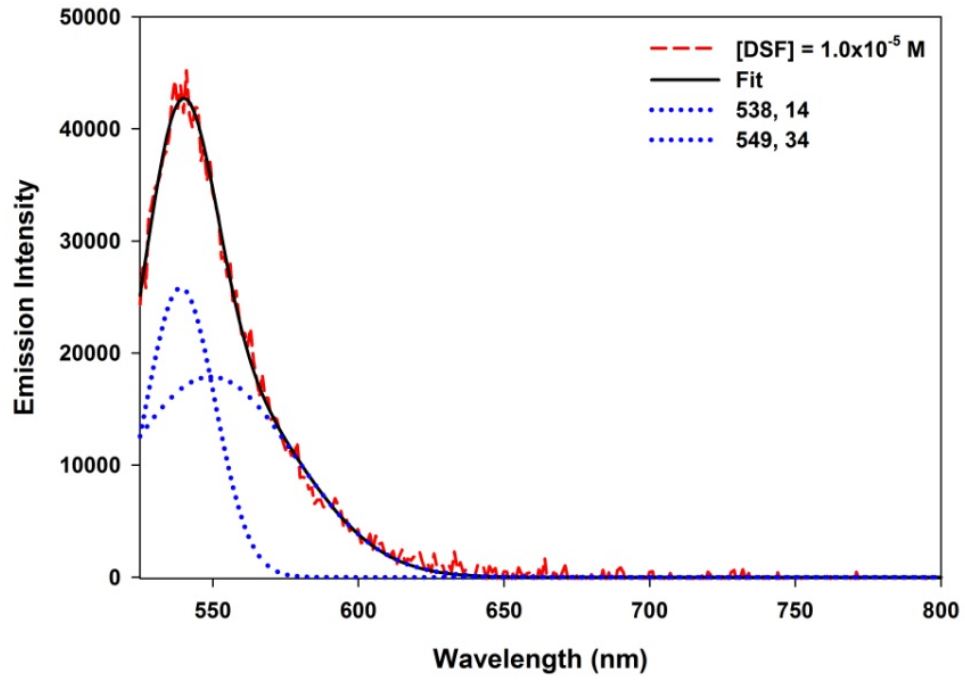

Figure S6. Deconvoluted emission spectra of films cast from different DSF concentrations. Legend shows peak position (nm) and full width at half max (nm).

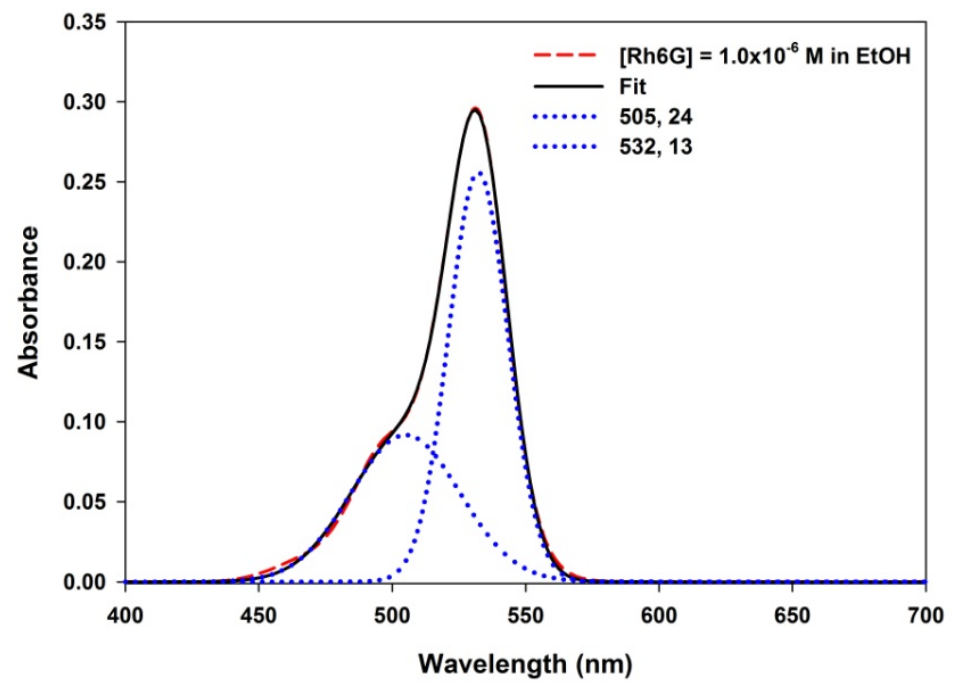




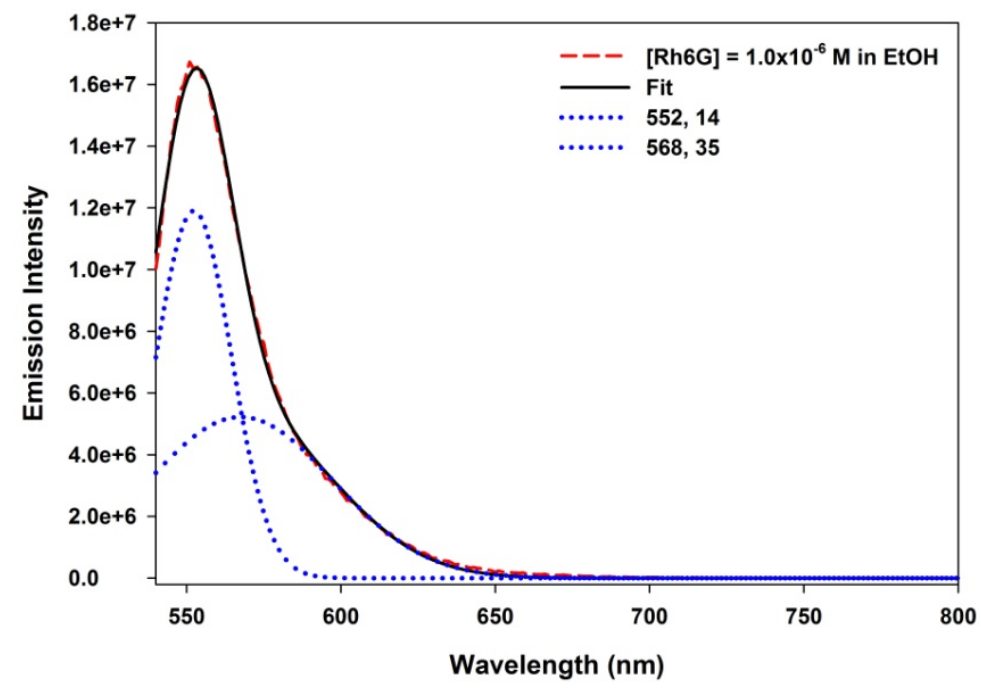

Figure S7. Deconvoluted spectra of $[\mathrm{Rh} 6 \mathrm{G}]=1.0 \times 10^{-6} \mathrm{M}$ in EtOH. Top: absorbance, Bottom: emission. Legend shows peak position (nm) and full width at half max (nm).

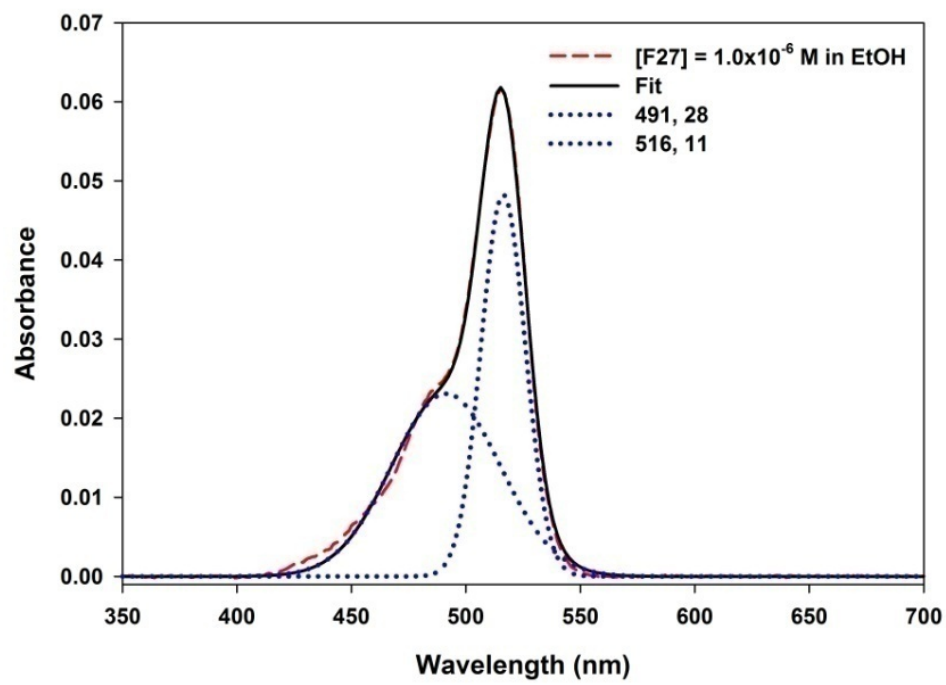




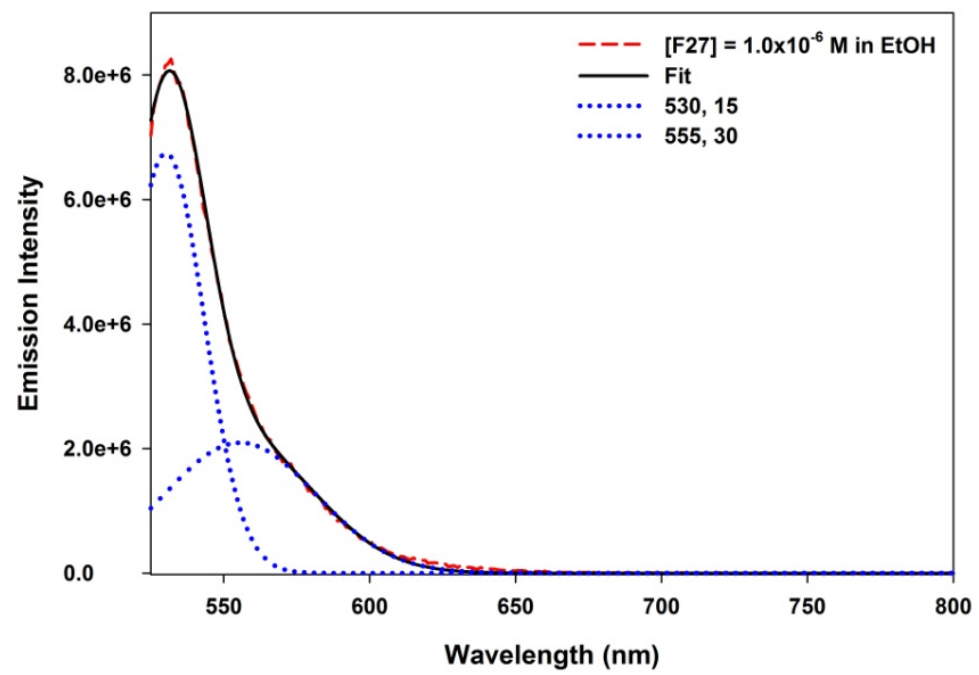

Figure S8. Deconvoluted spectra of $[\mathrm{F} 27]=1.0 \times 10^{-6} \mathrm{M}$ in EtOH. Top: absorbance, Bottom: emission. Legend shows peak position (nm) and full width at half max (nm).

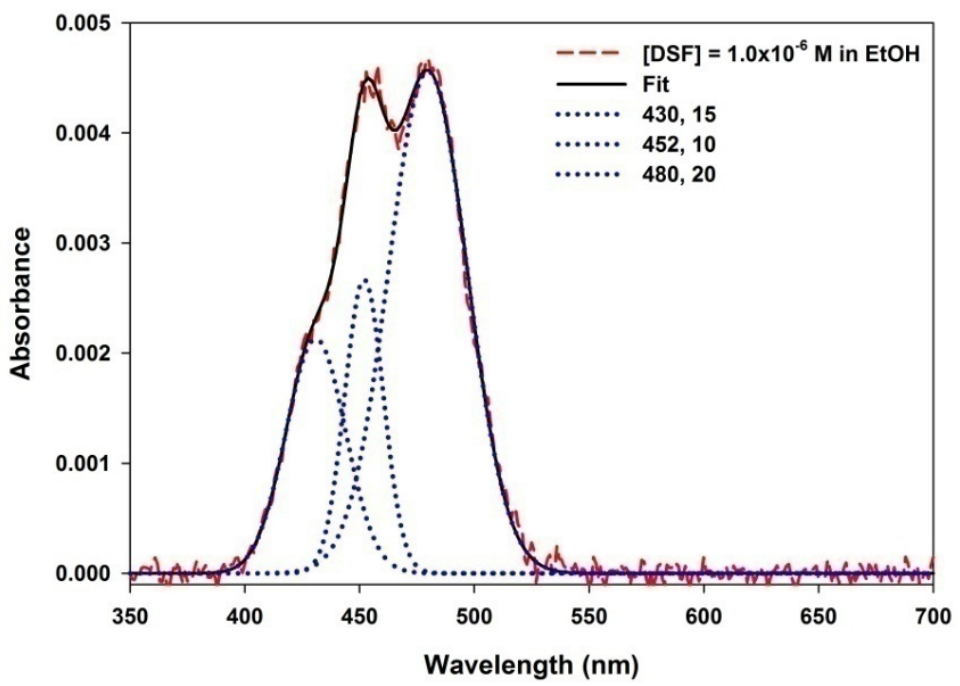




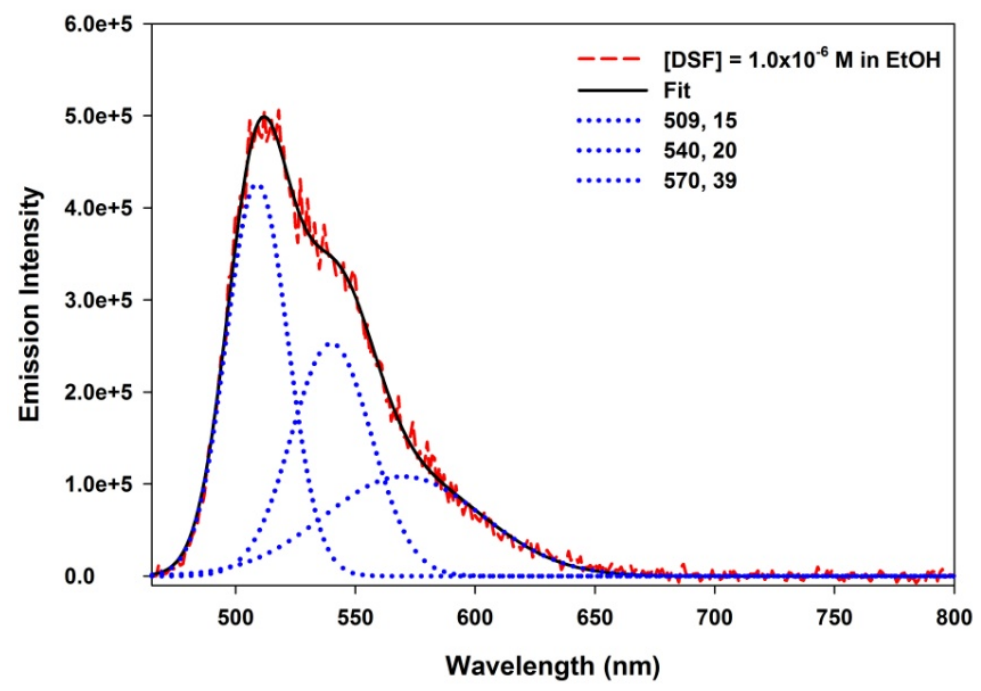

Figure S9. Deconvoluted spectra of $[\mathrm{DSF}]=1.0 \times 10^{-6} \mathrm{M}$ in EtOH. Top: absorbance, Bottom: emission. Legend shows peak position (nm) and full width at half max (nm). 


\begin{tabular}{|c|c|c|c|c|c|}
\hline Dye & Peak & $\begin{array}{c}\text { Absorbance } \\
\left(\lambda_{\max }, \Gamma\right) \\
(\mathrm{nm})\end{array}$ & $\begin{array}{c}\text { Concentration } \\
\text { Range (M) }\end{array}$ & $\begin{array}{c}\text { Emission } \\
\left(\lambda_{\max }, \Gamma\right) \\
(\mathrm{nm})\end{array}$ & $\begin{array}{c}\text { Concentration } \\
\text { Range (M) }\end{array}$ \\
\hline \multirow{5}{*}{ Rh6G } & 1 & 480,18 & $1 \times 10^{-3}-1 \times 10^{-5}$ & 560,25 & $1 \times 10^{-3}-1 \times 10^{-5}$ \\
\hline & 2 & 511,20 & $1 \times 10^{-3}-1 \times 10^{-5}$ & 605,22 & $1 \times 10^{-3}-1 \times 10^{-5}$ \\
\hline & 3 & 548,12 & $1 \times 10^{-3}-1 \times 10^{-5}$ & 644, 36 & $1 \times 10^{-3}-1 \times 10^{-5}$ \\
\hline & 4 & 584,12 & $1 \times 10^{-3}$ & - & - \\
\hline & 5 & 455,12 & $1 \times 10^{-3}$ & - & - \\
\hline \multirow{4}{*}{ F27 } & 1 & 480,14 & $1 \times 10^{-3}-1 \times 10^{-5}$ & 538,17 & $1 \times 10^{-3}-1 \times 10^{-5}$ \\
\hline & 2 & 500,18 & $1 \times 10^{-3}-1 \times 10^{-5}$ & 573,25 & $1 \times 10^{-3}-1 \times 10^{-5}$ \\
\hline & 3 & 520,12 & $1 \times 10^{-3}-1 \times 10^{-5}$ & - & - \\
\hline & 4 & 460,22 & $1 \times 10^{-3}, 1 \times 10^{-4}$ & - & - \\
\hline \multirow{6}{*}{ DSF } & 1 & 430,8 & $1 \times 10^{-3}-1 \times 10^{-5}$ & 538,15 & $1 \times 10^{-3}-1 \times 10^{-5}$ \\
\hline & 2 & 455,20 & $1 \times 10^{-3}-1 \times 10^{-5}$ & 548,34 & $1 \times 10^{-3}-1 \times 10^{-5}$ \\
\hline & 3 & 475,16 & $1 \times 10^{-3}-1 \times 10^{-5}$ & - & - \\
\hline & 4 & 505,17 & $1 \times 10^{-3}-1 \times 10^{-5}$ & - & - \\
\hline & 5 & 520,26 & $1 \times 10^{-3}, 1 \times 10^{-4}$ & - & - \\
\hline & 6 & 574,16 & $1 \times 10^{-3}$ & - & - \\
\hline
\end{tabular}

Table S1. Deconvolution parameters for the absorbance and emission spectra of dye thin films on PS. $\lambda_{\max }$ represent the peak position and $\Gamma$ is the FWHM. Concentration range indicates the range in which the corresponding peak was required to fit the spectrum of the film cast from that concentration. 